UNIVERSITÉ DE SHERBROOKE

Étude comparative de la métasynthèse et de la méta-analyse qualitative

par

Vincent Beaucher

Mémoire présenté à la Faculté d'éducation en vue de l'obtention du grade de

Maître es arts (M.A.)

Maîtrise en sciences de l'éducation

Mai 2007

(C) Vincent Beaucher 2007

$V-702$ 


$\begin{array}{ll}\begin{array}{l}\text { Library and } \\ \text { Archives Canada }\end{array} & \begin{array}{l}\text { Bibliothèque et } \\ \text { Archives Canada }\end{array} \\ \begin{array}{l}\text { Published Heritage } \\ \text { Branch }\end{array} & \begin{array}{l}\text { Direction du } \\ \text { Patrimoine de l'édition }\end{array} \\ \begin{array}{l}\text { 395 Wellington Street } \\ \text { Ottawa ON K1A 0N4 }\end{array} & \begin{array}{l}\text { 395, rue Wellington } \\ \text { Ottawa ON K1A ON4 } \\ \text { Canada }\end{array} \\ \end{array}$

Your file Votre référence ISBN: 978-0-494-31352-7 Our file Notre référence ISBN: 978-0-494-31352-7

NOTICE:

The author has granted a nonexclusive license allowing Library and Archives Canada to reproduce, publish, archive, preserve, conserve, communicate to the public by telecommunication or on the Internet, loan, distribute and sell theses worldwide, for commercial or noncommercial purposes, in microform, paper, electronic and/or any other formats.

The author retains copyright ownership and moral rights in this thesis. Neither the thesis nor substantial extracts from it may be printed or otherwise reproduced without the author's permission.
AVIS:

L'auteur a accordé une licence non exclusive permettant à la Bibliothèque et Archives Canada de reproduire, publier, archiver, sauvegarder, conserver, transmettre au public par télécommunication ou par l'Internet, prêter, distribuer et vendre des thèses partout dans le monde, à des fins commerciales ou autres, sur support microforme, papier, électronique et/ou autres formats.

L'auteur conserve la propriété du droit d'auteur et des droits moraux qui protège cette thèse. $\mathrm{Ni}$ la thèse ni des extraits substantiels de celle-ci ne doivent être imprimés ou autrement reproduits sans son autorisation.
In compliance with the Canadian

Privacy Act some supporting forms may have been removed from this thesis.

While these forms may be included in the document page count, their removal does not represent any loss of content from the thesis.
Conformément à la loi canadienne sur la protection de la vie privée, quelques formulaires secondaires ont été enlevés de cette thèse.

Bien que ces formulaires aient inclus dans la pagination, il n'y aura aucun contenu manquant. 


\section{Étude comparative de la métasynthèse et de la méta-analyse qualitative par Vincent Beaucher}

\section{RÉSUMÉ}

Ce mémoire fait le point sur une méthode d'analyse secondaire de recherches qualitatives, la métasynthèse. Pour ce faire, nous nous penchons sur les écrits de plusieurs auteurs et nous tentons d'établir des consensus, par exemple, sur sa définition, ses buts, ses composantes, ses limites. Avant d'entrer dans le coeur de la métasynthèse, nous abordons brièvement la méta-analyse qualitative, une méthode d'analyse secondaire plus connue et plus utilisée. Cela permet de mettre en relief les approches d'analyse secondaire en qualitatif et surtout de dégager les différences entre la méta-analyse qualitative et la métasynthèse. Ensuite, l'analyse des textes sur le sujet permet de décrire la métasynthèse. Il ressort que ses finalités sont de produire une nouvelle compréhension ou une théorie nouvelle par rapport à un phénomène. Le consensus n'est cependant pas atteint chez les auteurs sur le potentiel de la métasynthèse, de plus amples réflexions et écrits se révélant nécessaires. 


\section{UNIVERSITÉ DE SHERBROOKE \\ Faculté d'éducation}

Étude comparative de la métasynthèse

et de la méta-analyse qualitative

Vincent Beaucher

a été évalué par un jury composé des personnes suivantes :
M. Jacques Joly, Ph.D.
Président

Mme France Jutras, Ph. D.

Directrice de recherche

M. Enrique Correa Molina, Ph.D. Autre membre du jury

Mémoire accepté le : 


\section{SOMMAIRE}

Le mémoire que nous proposons est de nature « méthodologique », c'est-à-dire qu'il traite de méthodes de recherches. Plus spécifiquement, la méta-analyse qualitative et la métasynthèse y sont abordées. Plus encore, c'est surtout sur la métasynthèse que s'est effectué l'exercice. Le résultat en est un article qui présente un bref survol de la métaanalyse qualitative à des fins de comparaison, mais surtout une analyse plus en profondeur de la métasynthèse, notre objet d'intérêt.

$\mathrm{Au}$ départ, l'étude envisagée se voulait un retour analytique sur différentes expériences coopératives telles que vécues par des enseignants en contexte d'apprentissage coopératif. Nous voulions évaluer la relation enseignant-élève dans un tel contexte. Cette recherche devait se faire à partir d'écrits; il s'agissait en fait d'effectuer des analyses secondaires de recherches qualitatives. Or, il existe relativement peu d'informations sur d'éventuelles approches ayant cette raison d'être. Nous avons donc décidé de réorienter notre recherche et de nous appliquer à dégager les caractéristiques d'une de ces formes d'analyse secondaire: la métasynthèse. Pourquoi celle-ci en particulier? Essentiellement pour deux raisons: premièrement, elle est entièrement de nature qualitative, ce qui n'est pas toujours le cas pour d'autres méthodes d'analyse secondaire comme par exemple, la métaanalyse qualitative; deuxièmement, avec l'absence d'information à son sujet en sciences de l'éducation, nous jugions intéressant et pertinent de nous y intéresser.

Avant d'en arriver à traiter de la méta-analyse qualitative et surtout de la métasynthèse, nous avons établi le rationnel de la recherche, ce qui a consisté à revenir sur le comment et le pourquoi faire de la recherche en éducation, les éléments qui sous-tendent la recherche tels les paradigmes, les choix méthodologiques à poser et finalement les niveaux de la recherche. C'est à ce stade que sont devenues évidentes la possibilité et surtout la pertinence de faire de la recherche "secondaire », différente de la recherche " primaire ». Avec la multitude d'études primaires qui sont complétées chaque année, il 
devient de plus en plus opportun de réanalyser ces études, ce que font les approches d'analyse secondaire de recherches qualitatives. Cela se fait déjà depuis un certain temps en recherche quantitative, souvent par l'entremise de la méta-analyse. Cependant, force est de constater que ce n'est pas une habitude en recherche qualitative.

Appuyant notre argumentaire sur la nécessité de développer les connaissances sur les approches d'analyse secondaire et tout spécialement la métasynthèse, nous avons procédé à une recension pour trouver de l'information à son sujet. Nous n'avons pu que constater son absence générale dans les manuels de méthodologie de la recherche. Suite à de multiples recherches, nous avons toutefois pu mettre la main sur un article en sciences infirmières qui traitait de la métasynthèse, le premier d'un petit nombre sur lequel nous nous sommes basé pour réaliser une analyse de contenu dans le but de déterminer les grandes caractéristiques de la métasynthèse: sa définition, ses caractéristiques, ses buts, son fonctionnement, ses variantes, ses composantes et ses limites. Nous avons effectué une analyse similaire, quoique beaucoup moins approfondie, sur la méta-analyse qualitative dans le but de faire ressortir les similarités, mais surtout les différences, entre ces deux formes d'analyse secondaire. Il est à noter qu'il est plutôt facile de trouver des renseignements sur la méta-analyse qualitative, d'où le fait de nous être limité dans notre discussion à son sujet.

Plusieurs points ressortent de notre recherche, un des plus importants étant que la métasynthèse se veut une méthode qui permet de mettre en commun les résultats de plusieurs études primaires qualitatives et d'en faire émerger un aspect qui, par la force du nombre et par la rigueur de l'analyse, sera représentatif d'une « nouvelle » réalité. Il ne s'agit pas de simplement additionner, par exemple, le nombre de fois qu'un concept apparaît dans les études analysées, mais plutôt de remettre en contexte tous les concepts, ou ceux jugés pertinents, en jetant un regard critique sur ce qui a amené à l'émergence de ces concepts. Souvent, la métasynthèse prendra la forme d'une métaétude, ce qui impliquera l'opérationnalisation d'une métathéorie (retour sur les théories sous-jacentes aux études primaires), d'une métaméthode (retour sur les méthodes employées dans les études 
primaires) et d'une méta-analyse des données (retour sur les résultats). Plus complexe qu'il n'y paraît à première vue, une bonne expertise en méthodologie est requise pour mener à bien ce type de métasynthèse. D'ailleurs, une équipe de chercheurs peut s'avérer nécessaire, ou du moins, bien utile. Par ailleurs, notre étude sur la métasynthèse nous a éclairé sur le fait qu'il existe plusieurs formes de métasynthèse, parfois moins exigeante que celle de type métaétude, mais dont l'objectif demeure essentiellement d'exposer l'existence d'une réalité donnée à un phénomène particulier. Il demeure cependant un fait: le chercheur désirant utiliser la métasynthèse sur un corpus d'études sera définitivement plongé dans l'univers qualitatif, appelé à déconstruire, décontextualiser, interpréter, recontextualiser et plus encore.

Le constat final de notre recherche est le suivant : la métasynthèse est une approche qui mérite qu'on s'y attarde et il existe un besoin criant de clarification de ce concept. De plus en plus, la pertinence de mettre en oeuvre des approches d'analyse secondaire de recherches qualitatives sera démontrée. Alors que la recherche qualitative est encore relativement jeune, il viendra un moment où la quantité d'études réalisées justifiera une réflexion et surtout un retour sur ce qui a été trouvé. Or, il faudra à ce moment-là disposer de méthodes d'analyse secondaire dont l'utilisation sera concrètement à la portée des chercheurs, mais surtout dont les protocoles seront bien définis, solides et valides. Nous avons pu constater qu'à l'heure actuelle, il y a lacune à ce sujet et pas uniquement par rapport à la métasynthèse. En effet, c'est le cas pour l'ensemble de ces approches, dont une partie est présentée dans le cadre conceptuel de ce mémoire. Nous espérons donc voir se réaliser de plus amples recherches en la matière dans un futur rapproché; c'est toute la communauté scientifique qui en sortirait grandie. 


\section{TABLE DES MATIÈRES}

SOMMAIRE . .3

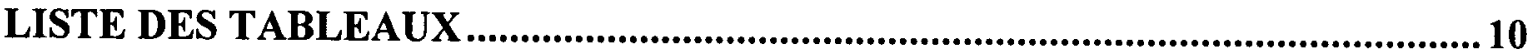

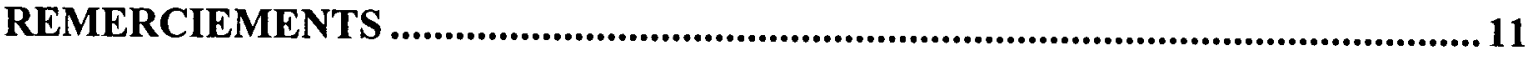

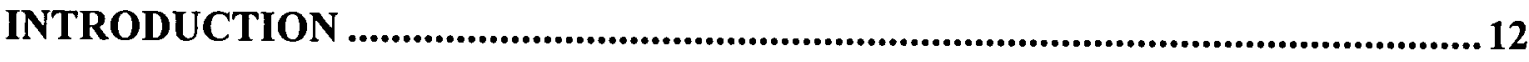

CHAPITRE UN LA PROBLÉMATIQUE ................................................................

1. LE RATIONNEL DE LA RECHERCHE EN ÉDUCATION.................................................15

1.1 Le développement de la connaissance en éducation ................................... 16

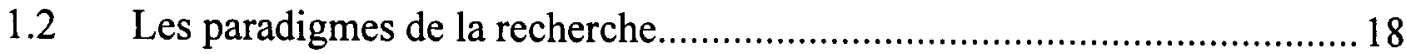

1.3 Les choix méthodologiques en recherche scientifique ..............................21

1.4 Les stratégies d'analyse secondaire de recherches .....................................27

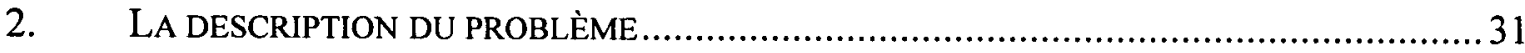

3. LA FORMULATION DE LA QUESTION GÉNÉRALE DE RECHERCHE …..............................32

4. LA RECENSION DES ÉCRITS SUR LA MÉTASYNTHÈSE ................................................ 32

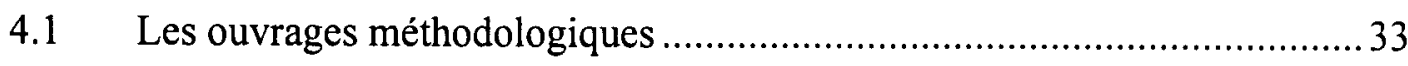

4.2 Les articles en sciences humaines, sociales et de l'éducation .......................35

4.3 Les articles disponibles dans d'autres domaines ........................................... 36

5. LA RECENSION DES ÉCRITS SUR LA MÉTA-ANALYSE QUALITATIVE .............................37

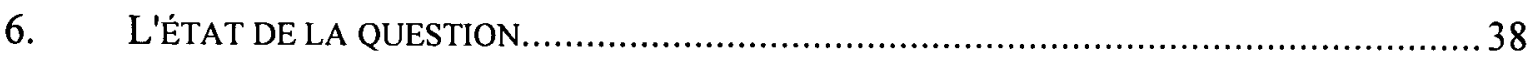

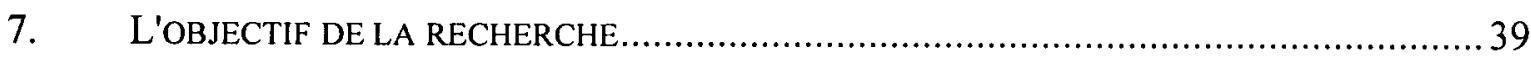

CHAPITRE DEUX LE CADRE CONCEPTUEL............................................................40

1. LES SIMILITUDES ENTRE LES SCIENCES INFIRMIÈRES ET DE L'ÉDUCATION ...................40

2. LES MÉTHODES AYANT POUR OBJECTIF LA SYNTHÈSE QUALITATIVE...........................44

2.1 Un exemple en éducation.................................................................... 4

2.2 Constat en rapport aux méthodes de synthèse.............................................. 48

3. LES DIMENSIONS DESCRIPTIVES PROPRES À UNE MÉTHODE DE RECHERCHE ................48

CHAPITRE TROIS LA MÉTHODOLOGIE .........................................................51 


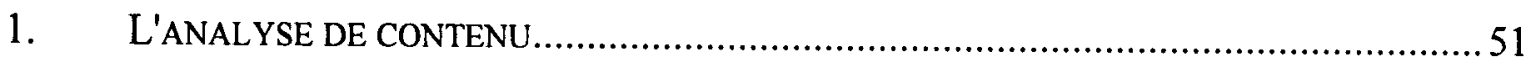

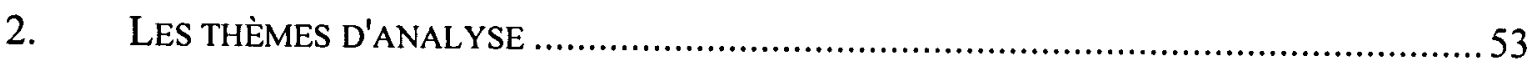

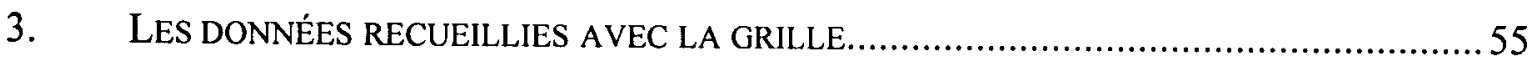

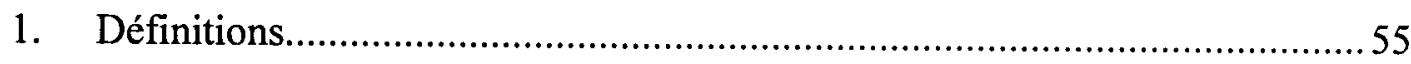

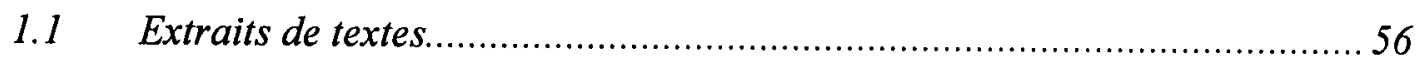

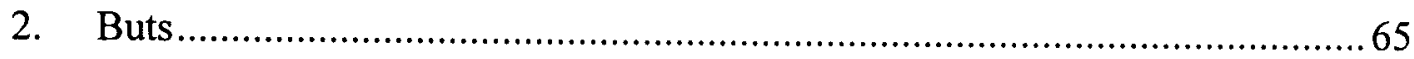

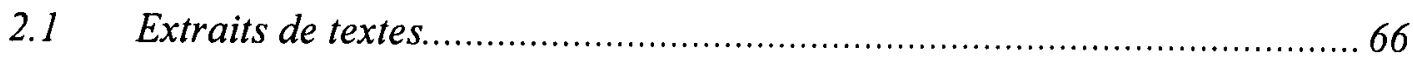

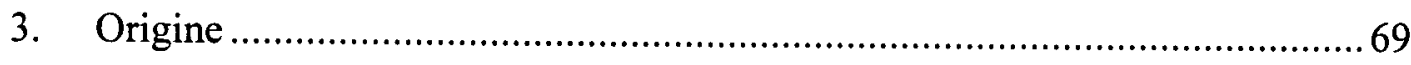

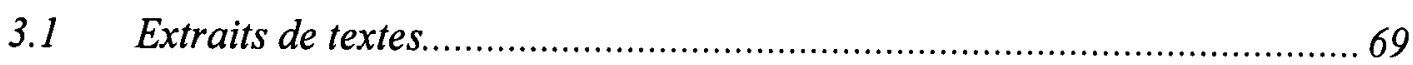

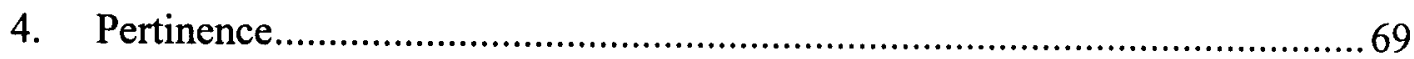

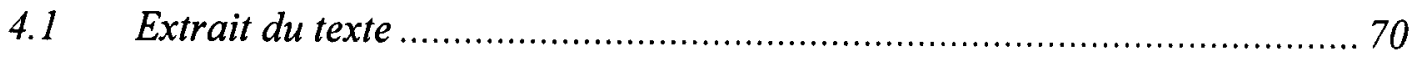

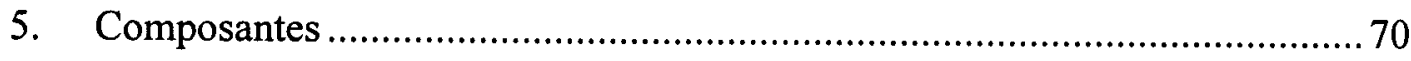

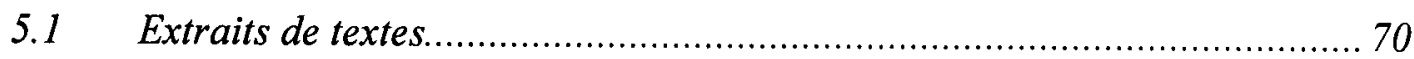

6. Étapes et procédures............................................................................ 72

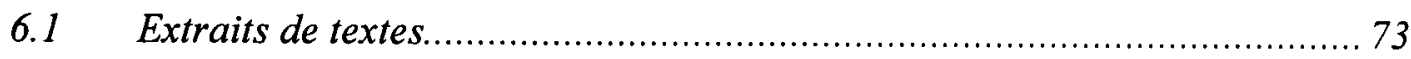

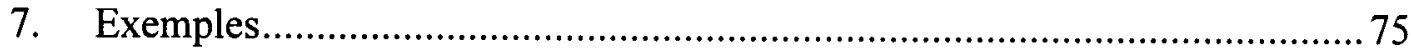

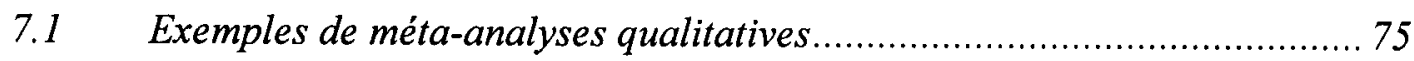

7.2 Exemples de métasynthèses................................................................. 76

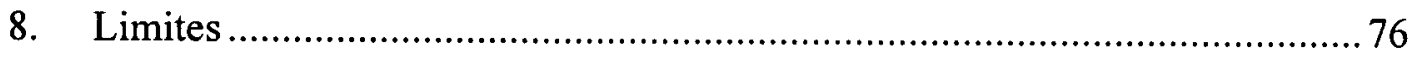

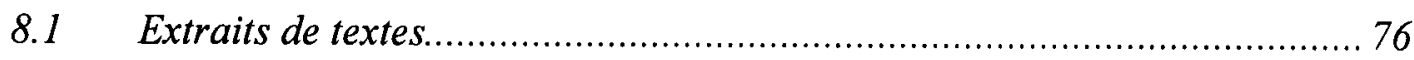

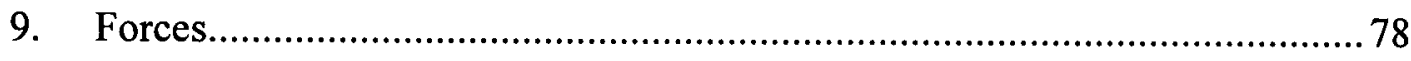

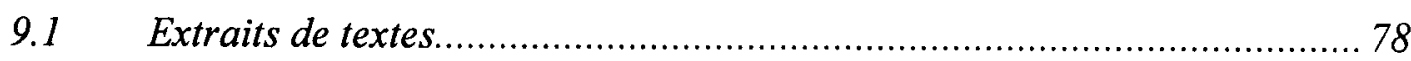

4. LA STRATÉGIE DE PRÉSENTATION DES RÉSULTATS ...............................................79

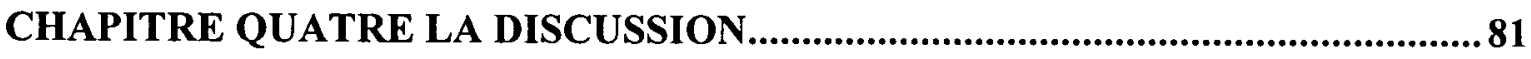

RÉSUMÉ

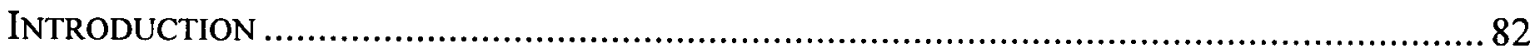

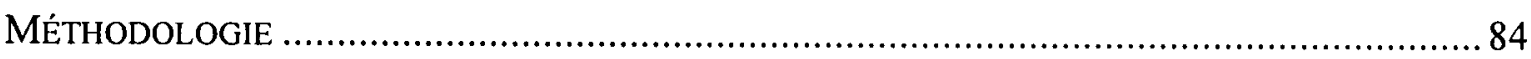

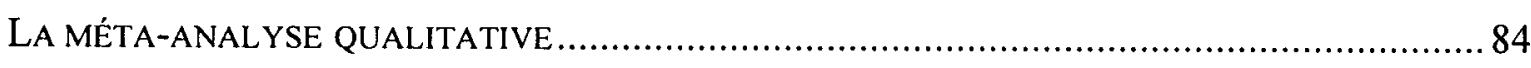

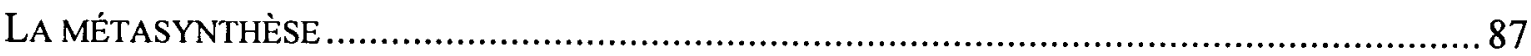


Qu'est-ce que la métasynthèse? 87

Pourquoi utiliser la métasynthèse? .............................................................. 91

Comment faire une métasynthèse?............................................................ 95

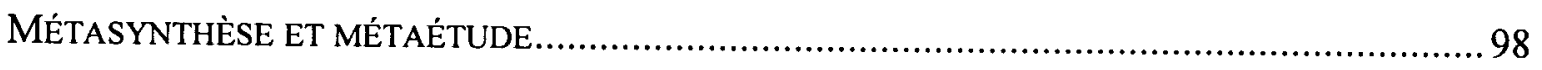

Le lien ambigu entre la métaétude et la métasynthèse ...................................... 98

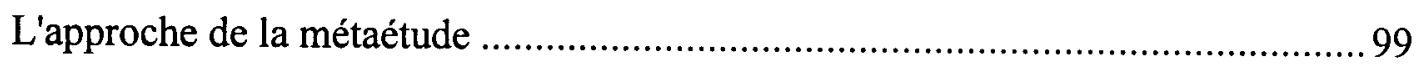

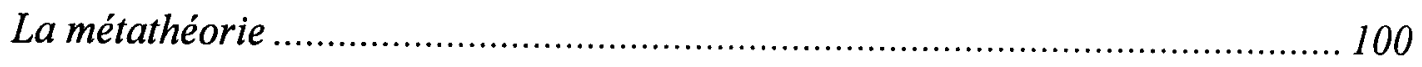

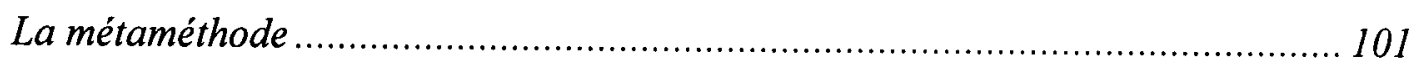

La méta-analyse de données ................................................................. 102

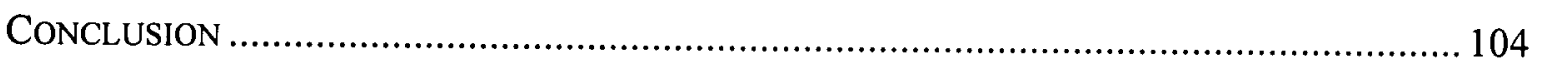

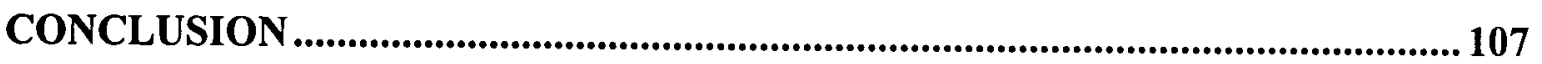

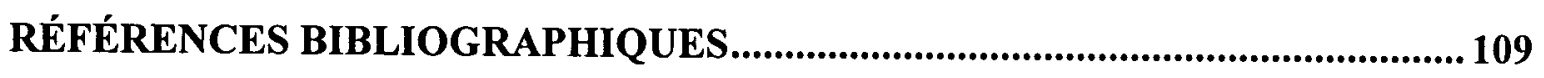

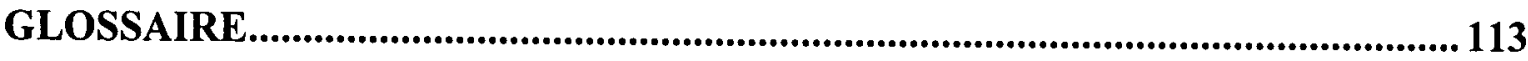

ANNEXE A Les livres Qui CONCERNENT LA RECHERCHE QUALITATIVE SE RETROUVANT

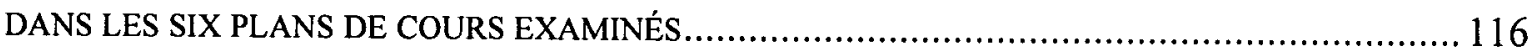

ANNEXE B LES LIVRES QUI CONCERNENT LA RECHERCHE QUALITATIVE SÉLECTIONNÉS À LA BIBLIOTHĖQUE POUR COMPLÉMENTER L'ÉCHANTILLON PRÉSENTÉ À L'ANNEXE A.............. 119

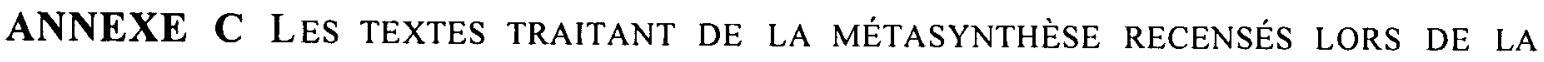
RECHERCHE DOCUMENTAIRE ET SUBSÉQUEMMENT ÉTUDIÉS LORS DE L'ANALYSE DE CONTENU

ANNEXE D LES TEXTES TRAITANT DE LA MÉTA-ANALYSE QUALITATIVE RECENSÉS LORS DE LA RECHERCHE DOCUMENTAIRE ET SUBSÉQUEMMENT ÉTUDIÉS LORS DE L'ANALYSE DE

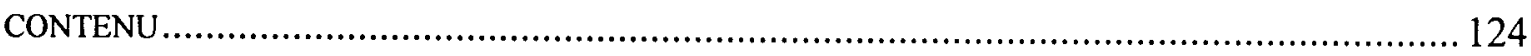

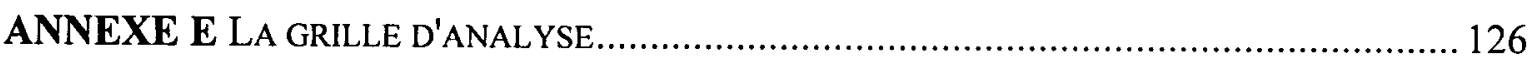

ANNEXE F RÔLES DES COAUTEURS DE L'ARTICLE PRÉSENTÉ DANS LE CHAPITRE QUATRE

ANNEXE G ACCUSÉ DE RÉCEPTION DE LA REVUE CONTACTÉE 
ANNEXE H NORMES DE PRÉSENTATION DE LA REVUE CONTACTÉE 132 


\section{LISTE DES TABLEAUX}

Tableau 1 Comparaison des pôles de la recherche qualitative et quantitative (Paillé, 2003) p. 25

Tableau 2 Définition d'une méthode de recherche (Van der Maren, 1996) p. 26

Tableau 3 Présentation sommaire de méthodes d'analyse secondaire qualitatives.. p. 45

Tableau 4 Thèmes descriptifs retenus aux fins d'analyse de la métasynthèse ......... p. 50

Tableau 5 Étapes d'une analyse de contenu (L'Écuyer, 1988) p. 54 


\section{REMERCIEMENTS}

L'auteur de ces lignes tient à remercier quelques personnes qui ont contribué à faire avancer cette recherche. Tout d'abord, par ses conseils et surtout ses encouragements, $M$. Pierre Paillé, Ph.D, professeur à l'Université de Sherbrooke et expert en recherche qualitative, a été une source inspirante en plus de participer de façon constructive à l'évaluation du projet de recherche. Ensuite, M. Jacques Joly, Ph.D., professeur à l'Université de Sherbrooke s'est également prêté au jeu de l'évaluation du projet de recherche et ses remarques fort judicieuses ont réorienté quelque peu la recherche et permis d'accroître sa solidité. Finalement, tout le travail accompli n'aurait pu être possible sans le dévouement continu de Mme France Jutras, Ph.D., professeure à l'Université de Sherbrooke et directrice de ce mémoire de maîtrise. D'une très grande disponibilité en personne ou de façon virtuelle, ses conseils, sa motivation, ses (re)lectures et ses corrections ont été d'une aide très enrichissante et hors du commun. 


\section{INTRODUCTION}

Ce mémoire de maîtrise en sciences de l'éducation a pour but de présenter une méthode d'analyse secondaire de données issues de recherches qualitatives, la métasynthèse. Celle-ci sera comparée à la méta-analyse qualitative, méthode de forme similaire, mais plus connue. La raison qui nous pousse à nous intéresser à la métasynthèse vient du fait que cette dernière est plutôt méconnue dans le domaine de l'éducation. C'est à cet égard que la présente recherche, en plus d'un court volet comparatif, s'attarde tout spécialement à expliciter la métasynthèse.

Pour comprendre l'intérêt d'effectuer notre recherche, nous procédons en grandes étapes. Tout d'abord, le chapitre 1 traite de la problématique. À l'intérieur de celle-ci, dans un premier temps, nous mettons en évidence le rationnel de la recherche en éducation pour bien situer l'objet de recherche et la problématique dans laquelle il s'inscrit. Dans un deuxième temps, nous présentons le problème soulevé. Dans un troisième temps, la question générale de recherche est exprimée. Dans un quatrième temps, nous tentons de répondre à cette question générale. Pour ce faire, nous voulons dégager des écrits ce qu'ils peuvent nous révéler sur la méta-analyse qualitative et surtout sur la métasynthèse. Nous concluons le premier chapitre par l'objectif de la recherche qui est de comparer la métasynthèse et la méta-analyse qualitative dans l'optique de les caractériser et de les distinguer. Dans le deuxième chapitre, le cadre conceptuel est abordé de manière à clarifier les décisions qui nous amènent à la méthodologie de la recherche, soit l'analyse de contenu. Le troisième chapitre renferme ainsi la méthodologie utilisée pour atteindre l'objectif de la recherche. Les résultats de notre recherche se retrouvent dans le quatrième chapitre, sous la forme d'un article scientifique soumis à la revue Recherches qualitatives.

Avant d'aborder la problématisation de notre recherche, prenons un instant pour comprendre d'où vient l'idée de se pencher sur deux méthodes de recherche, la métaanalyse qualitative et plus particulièrement la métasynthèse. Le fait d'aborder la 
métasynthèse pour cette recherche est apparu lors d'une démarche d'examen des méthodes d'analyse de résultats déjà publiés. L'idée de départ de notre recherche consistait à effectuer une étude scientifique et rigoureuse d'analyse secondaire de données qualitatives. Plus spécifiquement, nous désirions trouver, à travers diverses études sur l'expérience de la coopération en classe, des éléments nous permettant de faire le point sur la relation maîtreélève dans un environnement utilisant la pédagogie de la coopération au secondaire. Pour ce faire, la possibilité de recourir à des études déjà publiées, sur un thème bien précis, est apparue comme une avenue intéressante à emprunter. Cependant, une telle recherche devait aller plus loin que la simple synthèse, aussi étendue fût-elle. En discutant avec des chercheurs en éducation et en consultant des livres portant sur la recherche en éducation, le concept de la méta-analyse a été dégagé. De prime abord, la proposition était pertinente, car le regroupement de résultats d'études complétées est au coeur de cette forme de recherche. Cependant, à la suite de quelques lectures préliminaires, il est vite apparu que ce type de recherche ne correspondait pas tout à fait à nos visées. Or, en cherchant des informations complémentaires, un court texte, Ecological Triangulation: An Approach for Qualitative Meta-Synthesis (Banning, sans date), apporta un angle nouveau et surtout très intéressant quant à la façon d'agglomérer des résultats de recherches qualitatives, ce que nous cherchions à faire. Par son aspect innovateur, de même que par ses finalités rejoignant, et dépassant même, les buts de notre recherche immédiate, il a été décidé d'emprunter cette avenue.

Avant de débuter par la présentation du premier chapitre, des précisions s'imposent. Puisque les objets de cette recherche sont des méthodes de recherche et d'analyse, le lecteur trouvera dans le texte plusieurs termes techniques propres à la recherche scientifique. Or, après plusieurs consultations de livres, de manuels de référence et autres sources pertinentes au domaine, il n'est toujours pas aisé de clairement définir et situer, sans ambiguité, certains des concepts utilisés. Nous faisons tout ce qui est nécessaire pour offrir la vision conceptuelle la plus juste possible, malgré les différences qu'on retrouve chez les divers auteurs. C'est pourquoi les termes scientifiques sont définis dans le texte au 
fur et à mesure que nous les utilisons et ils sont intégrés, tels que définis dans le texte, au glossaire qu'on retrouve à la fin du mémoire. 


\section{CHAPITRE UN \\ LA PROBLÉMATIQUE}

Le premier chapitre a pour but d'expliciter la problématique entourant nos deux objets de recherche, deux méthodes de recherche de nature qualitative. Nous abordons alors la recherche en éducation comme un contexte, dans l'optique de situer les méthodes de recherche présentement utilisées par les chercheurs du domaine, mais également pour faire valoir la pertinence du type d'étude que nous entreprenons ici. Pour ce faire, il est question des concepts fondamentaux de la recherche et des éléments qui font de cette recherche un acte scientifique. À la suite de cette mise en contexte, nous abordons la problématique spécifique. En découle une question générale de recherche qui nous a amené à réaliser une recension des écrits. Le chapitre se termine sur l'objectif de la recherche.

\section{LE RATIONNEL DE LA RECHERCHE EN ÉDUCATION}

Procéder à un questionnement sur une forme de recherche n'est jamais un processus vain, car cela permet de comprendre toujours un peu plus les différentes visions qui la composent, ses buts, ses caractéristiques, etc. Cependant, il convient d'être en mesure de positionner son objet d'étude dans le grand champ de la recherche scientifique qui supporte notre domaine de savoir. Cela amène souvent du même coup à exposer les raisons fondamentales d'effectuer de la recherche dans un champ. C'est là une des finalités de la présente section, en plus d'explorer les différentes ramifications qui composent la recherche dans son acte scientifique. Cela conduit à mettre en évidence le rationnel de la recherche et aussi de comprendre la situation qu'occupe nos objets d'étude. 


\subsection{Le développement de la connaissance en éducation}

Nous abordons ici la relation qui existe entre la recherche scientifique et l'éducation. Il s'agit de comprendre ce qui incite les chercheurs oeuvrant dans le monde de l'éducation à faire de la recherche, dans ses différentes formes et finalités. L'exercice effectué ne se veut pas une étude en profondeur de chacun des concepts qui seront présentés, mais davantage une volonté de dégager une compréhension sommaire, mais aussi complète que possible, des bases et orientations de la recherche scientifique en éducation ${ }^{1}$. À la suite de la lecture de cette section, les places de la méta-analyse de même que celle de la métasynthèse comme méthodes ayant des particularités fondamentales devraient être plus claires, tout comme pour le cas de la pertinence d'entreprendre l'étude que nous proposons. La pertinence de notre démarche est également reprise dans le cadre conceptuel.

L'éducation, comme toutes les sciences, se doit de progresser et d'innover. Pour ce faire, la recherche est utilisée pour trouver réponse aux nombreux questionnements des spécialistes du domaine sur des problèmes rencontrés aux niveaux pratique et théorique. En bref, « la raison majeure pour de la recherche en éducation est de développer de nouvelles connaissances à propos de l'enseignement, de l'apprentissage et de l'administration scolaire ${ }^{2}$ (Borg, Borg et Gall, 1989, p. 4, traduction libre). Cependant, la recherche éducative peut également se pencher sur ses propres agirs, sur la façon même dont sont issues les nouvelles connaissances. Ainsi, comme le mentionnent les mêmes auteurs, « un autre type de connaissance, d'un ordre différent de ceux-ci [description, prédiction, amélioration et explication] mérite une mention. En réfléchissant sur leur

\footnotetext{
${ }^{1}$ En effet, chacun des concepts abordés dans les paragraphes qui suivent pourrait faire l'objet d'une thèse à lui seul. De fait, les définitions, les compréhensions, les visions de tous ces thèmes divergent très souvent d'un auteur à un autre. En faire une étude approfondie demanderait par ailleurs une compréhension très poussée des fondements mêmes, de l'épistémologie de la recherche scientifiqque, de sa philosophie. Notre objectif consiste seulement à offrir une vision généraliste, mais aussi juste que possible, pour comprendre les liens qui unissent la métasynthèse et la recherche en éducation.

${ }^{2}$ "The major reason for educational research is to develop 'new knowledge' about teaching and learning and administration."
} 
travail, les chercheurs en éducation continuent de développer de nouvelles connaissances sur la façon de mettre au point et de conduire une recherche. $\nu^{3}$ (Ibid., p. 10) Or, c'est exactement ce que nous nous proposons de faire. Pour Van der Maren (1996), il s'agit alors de traiter de la question de la *méthodologie de la recherche ${ }^{4}$. Il définit ce concept comme l'ensemble « des études et des recherches à propos des méthodes de recherche et du discours produit par ces travaux. $»($ p. 112)

On s'en doute bien, pour conduire une étude sur la façon de faire de la recherche, au même titre que de faire une étude sur un quelconque sujet dans le cadre d'une recherche universitaire, il faut être en mesure de bien cadrer son objet de recherche (ici, la métasynthèse et la méta-analyse qualitative) dans un contexte qui lui est propre (la recherche). Dans cette optique, il est important de porter un regard critique et intéressé sur les bases des processus d'avancement du savoir, de la recherche scientifique. Ce contexte est donc important à cerner et nous pouvons déjà affirmer que son origine n'est pas récente. En fait, depuis des décennies, voire des siècles, différents protocoles de recherche ont été établis, mais jamais dans une forme que l'on pourrait qualifier de finale. Ces protocoles déterminent les différentes démarches de la recherche scientifique. Une recherche est donc dite scientifique quand il s'agit d'un " processus de cueillette systématique de données observables et vérifiables en vue de la connaissance et de la compréhension du monde " (Legendre, 2005, p. 1155). Mais, avant même la mise en oeuvre de la démarche concrète, la rècherche doit reposer sur des assises théoriques, idéologiques, paradigmatiques. C'est en ce sens que nous allons aborder les paradigmes de la recherche. Cette démarche a pour objectif de nous permettre de raffiner la compréhension de notre contexte de recherche.

\footnotetext{
${ }^{3}$ " One other type of knowledge, of a different order from these, deserves mention. In reflecting on their work, educational researchers keep developing new knowledge about how to design and conduct research."

${ }^{4} \mathrm{Il}$ est à noter que les concepts précédés d'un astérisque sont définis dans le texte et repris dans un glossaire, en annexe.
} 


\subsection{Les paradigmes de la recherche}

Les * paradigmes déterminent les grandes orientations de la recherche scientifique et les résultats obtenus par ces recherches ont du sens par rapport au paradigme qui a soutenu l'orientation du protocole de recherche (Guba, 1990). Le concept de paradigme n'est pas nécessairement clair pour tous, mais il s'avère primordial pour le chercheur scientifique. Les paragraphes qui suivent ont pour but de jeter un regard succinct, mais pertinent, sur la notion du paradigme.

Pour effectuer de la recherche scientifique, le chercheur ou la chercheuse doit impérativement être en mesure de se positionner face à son objet d'étude. En effet, il convient d'être en mesure d'évaluer ce qu'est la connaissance, le réel, la relation entre celui qui cherche et le savoir, de même que la manière dont un chercheur peut contribuer à développer du savoir. Ces éléments de questionnement sont présentés par Guba (1990) alors qu'il analyse les aspects caractérisant un paradigme. Guba considère que ces questions sont d'ordre *ontologique (soit la représentation, la compréhension de la connaissance, du réel), *épistémologique (soit la relation entre celui qui cherche et le savoir) et *méthodologique (soit le procédé de recherche). Il donne par ailleurs une définition - qu'il dit très personnelle - du concept de paradigme: « un système de base de croyances guidant des actions dans un contexte social ordinaire, ou encore en relation avec une recherche structurée $»^{5}$ (p. 17, traduction libre). Il est le premier à reconnaître l'ambivalence d'une définition du terme « paradigme», affirmant du même coup que « ce n'est pas surprenant parce que Thomas Kuhn, la personne responsable en grande partie de la popularité du concept, aurait lui-même utilisé ce terme d'au moins 21 différentes manières, si Masterman (1970) est crédible $"^{6}$ (Ibid.). Ainsi, une définition différente nous est proposée par Legendre (2005):

\footnotetext{
5 " [...] a basic set of beliefs that guides action, whether of the everyday garden variety or action taken in connection with a disciplined inquiry. "

${ }^{6}$ "I say it is not surprising because Thomas Kuhn, the person most responsible for bringing that concept into our collective awareness, has himself used the term no fewer than 21 different ways, if Masterman (1970) can be believed."
} 
Ensemble d'énoncés ayant fonction de prémisses, présentant une vision globale d'un domaine, facilitant la communication et l'évolution, situant l'étude des phénomènes concernés, guidant l'élaboration des théories et suggérant les pratiques appropriées. (p. 981)

Gardons l'idée qu'un paradigme consiste en un ensemble de valeurs qui orientent nos actions lors d'une recherche scientifique, pour demeurer dans le cadre qui nous intéresse. Toujours selon Guba, il existe quatre paradigmes à la base de la recherche scientifique. Prenons-les un à un, la compréhension de ceux-ci étant nécessaire pour situer l'objet de recherche qui nous intéresse.

Le premier paradigme, selon Guba, est le positivisme. Se basant sur une ontologie " réaliste ", ce paradigme soutient l'existence d'un réel dirigé par les lois naturelles. La généralisation est visée, résultant parfois en des lois de causalité. Selon l'épistémologie " dualiste/objectiviste » qui caractérise ce modèle, le chercheur peut et doit adopter une posture non interactive et distante de son objet de recherche, ce qui garantirait l'objectivité de la recherche. Pour arriver aux fins de l'étude, le chercheur positiviste utilisera une méthodologie « expérimentale/manipulative », pratiquant des tests empiriques dans des conditions très contrôlées pour confirmer ou infirmer les questions et/ou hypothèses de recherche. Nous reviendrons un peu plus loin sur le positivisme lorsqu'il sera question des méthodes quantitatives. Poursuivons avec le deuxième paradigme présenté par Guba.

Comme son nom l'indique, le postpositivisme est apparu après le positivisme, en quelque sorte pour contrer les lacunes décelées par certains chercheurs peu enclins à concevoir la rigidité du réel présenté par le paradigme positiviste. Il s'agirait en fait « d'une version modifiée du positivisme $\|^{7}$ (Ibid., p. 20, traduction libre). Selon l'ontologie " réaliste critique " de ce modèle, il y a existence d'une réalité, sa compréhension ne pouvant cependant être totale (ce qui diffère du positivisme). L'épistémologie consisterait en de "l'objectivisme modifié », car même si l'objectivité est souhaitée et visée, elle est admise comme ne pouvant qu'être approximative. C'est la critique entretenue par le

\footnotetext{
${ }^{7}$ " Postpositivism is best characterized as a modified version of positivism."
} 
chercheur qui devient le principal gage de fiabilité de cet aspect. La méthodologie est de type « expérimental/manipulateur modifié », mettant l'emphase sur les multiples méthodes critiques disponibles. Par sa méthodologie, le postpositivisme tente de pallier les lacunes ou les limites du positivisme en effectuant des recherches dans des milieux plus naturels, avec davantage de méthodes qualitatives, se référant souvent à la théorie ancrée et réintroduisant le concept de découverte dans les enquêtes effectuées. Cependant, ces fondements prenant racine dans le positivisme portent encore son influence. Il ne s'agit donc pas d'un paradigme totalement indépendant du positivisme.

Guba explique ensuite les bases d'un troisième paradigme, soit la théorie critique, paradigme qu'il préférerait nommer " enquête idéologiquement orientée » (« ideologicallly oriented inquiry ») (p. 23). Pour mieux présenter ce paradigme, citons l'auteur:

[La théorie critique inclut] le néo-marxisme, le matérialisme, le féminisme, le freireisme, l'enquête participative et d'autres idéologies similaires, avec la critique théorique elle-même. Ces visions sont réunies de façon pertinente, car elles se rejoignent en rejetant l'allégation de l'objectivité [du chercheur] face à ses valeurs (value freedom) proposée par les positivistes (et largement perpétuée par les postpositivistes). ${ }^{8}$ (p. 23, traduction libre)

Pour résumer le paradigme de la théorie critique, on peut souligner que l'aspect ontologique est d'ordre « réaliste critique » (comme c'est le cas pour le postpositivisme), que l'épistémologie est "subjectiviste » et que la méthodologie est " dialogique et transformative $»$.

Le quatrième et dernier paradigme présenté par Guba est le constructivisme. De façon plus drastique que les adeptes de la théorie critique, les constructivistes renient les approches positivistes et postpositivistes, argumentant pour un remplacement de ces paradigmes, purement et simplement. Brièvement, notons que l'ontologie du

\footnotetext{
${ }^{8}$ * [...] including neo-Marxism, materialism, feminism, Freireism, participatory inquiry, and other similar movement as well as critical theory itself. These perspectives are properly placed together, however, because they converge in rejecting the claim of value freedom made by positivism (and largely continuing to be made by postpositivists). "
} 
constructivisme se veut « réaliste » (appuyant la thèse de l'existence d'une multiplicité de réalités), que l'épistémologie est « subjectiviste » (avec le concept d'une entité commune formée par l'interaction entre le chercheur et le participant à la recherche) et que, finalement, la méthodologie se veut « herméneutique et dialectique ».

Guba ayant présenté ces quatre paradigmes, d'autres auteurs en mentionnent d'autres, ou parfois les mêmes sous des appellations différentes. Mentionnons le paradigme naturaliste (Savoie-Zajc, 2003); les paradigmes compréhensif, subjectiviste et interprétatif (Mucchielli, 2003); les paradigmes participatif et pragmatique (Creswell, 2003). Il convient d'être au fait de l'existence de paradigmes alternatifs, ceux-ci rejoignant à divers degrés et/ou sur divers points les paradigmes de la théorie critique ou du constructivisme tels que présentés ci-haut, mais ayant tous comme point en commun de s'éloigner du positivisme. Quoiqu'il en soit, la raison de ce rappel quant aux différents paradigmes qui sous-tendent la recherche en éducation n'a pas pour but de déterminer lequel privilégier, mais plutôt pour bien voir qu'il existe plusieurs visions dans la recherche, dans les domaines de connaissances, et que ceux-ci ont un rôle primordial à jouer sur tout ce qui touche l'avancement des connaissances. Par exemple, et ce sera l'objet de la prochaine section, les caractéristiques des paradigmes déterminent souvent les approches de recherche pouvant servir aux fins des études inscrites à l'intérieur de l'un ou l'autre des paradigmes dont il vient d'être question.

\subsection{Les choix méthodologiques en recherche scientifique}

Si les paradigmes permettent de comprendre les grandes orientations idéologiques sur lesquelles se basent toutes les recherches, c'est que le fait d'en adopter un en particulier aura des incidences sur les théories utilisées, le but de la recherche et également sur les méthodes de recherche et les instruments utilisés pour collecter et analyser les données. Dans cette optique, il est pertinent de s'interroger sur les différentes approches de recherche. C'est ce dont il sera question dans les prochains paragraphes. 
Selon les auteurs, il existe essentiellement deux grandes «familles » en méthodologie de la recherche scientifique, deux choix méthodologiques qui s'offrent au chercheur: l'approche quantitative et l'approche qualitative. Depuis quelques années, par contre, une nouvelle approche a émergé, soit celle des méthodes mixtes. Comme son nom l'indique, elle est le résultat de la combinaison de méthodes quantitatives et qualitatives. Cette manière de faire trouve essentiellement son origine dans l'éclosion de nouveaux paradigmes alternatifs (Creswell, 2003). Dans le cadre de ce projet de recherche, nous allons nous attarder sur les deux approches les plus traditionnelles, ce qui n'empêche pas de cerner les fondements et attributs des méthodes mixtes. Nous voulons bien distinguer ces deux approches et les situer de façon somme toute schématique dans le cadre de la recherche scientifique. Pour les différencier, abordons dans un premier temps l'approche quantitative.

Dans Méthodes de recherche pour l'éducation (1996), Van der Maren présente les principaux éléments propres à *l'approche quantitative en recherche. Tout d'abord, les études prenant cette orientation sont généralement prescrites par des théories " descriptives », ou encore par des « métathéories formalisantes ». Les démarches utilisées seront de nature « expérimentale », " hypothético-déductive » ou encore « vérificative ». Finalement, les données analysées seront « métriques ». Notons que ces éléments décrivent une méthodologie se situant souvent dans le paradigme positiviste. En fait, disons plutôt que le paradigme positiviste emploiera presque exclusivement des méthodes reliées à l'approche quantitative pour analyser son réel. Bien que cela puisse sembler comme allant de soi, Van der Maren (1996) explique la logique du lien qui unit positivisme et quantitatif:

L'approche quantitative est plutôt concernée par les théories descriptives qui portent sur le comment des choses en exprimant des relations de dépendance fonctionnelle entre variables: elles identifient les éléments constitutifs de l'objet étudié, elles établissent la structure et l'évolution des relations entre ces éléments ainsi que les transformations que cet objet manifeste de façon concomitante ou consécutive aux modifications de son environnement. L'énonciation des théories descriptives implique donc l'observation d'états et de transformations d'états par suite de modifications produites sans intervention des chercheurs ou à la suite des manipulations effectuées par les chercheurs. (p. 88) 
Ce qu'il faut reconnaître ici, à la lecture de cette description, ce sont les liens entre l'approche quantitative et le paradigme positiviste. Dans un cas, on parle de dépendance fonctionnelle, et dans l'autre, de lois de causalité, ce qui revient essentiellement au même. Dans les deux cas, on aborde la distanciation du chercheur vis-à-vis son objet de recherche, ou du moins son objectivité s'il en est le manipulateur. L'on rejoint de façon très explicite ici la méthodologie expérimentale/manipulative propre au paradigme. Pour ces raisons, le lien entre ces deux concepts est pertinent. Quoiqu'il en soit, une nuance s'impose ici. Phillips et Burbules (2000), dans Postpositivism and educational research, discutent du positivisme dans l'optique d'introduire le postpositivisme. Ce faisant, ils apportent divers points sur lesquels il y a, selon eux, erreur d'interprétation et de compréhension du paradigme positiviste. L'un de ces points serait la fausse conception selon laquelle le chercheur positiviste se doit d'adhérer et d'utiliser la méthode scientifique comme procédure de recherche, tout comme le fait d'avoir recours à ce procédé ne fait pas automatiquement du chercheur un positiviste. Dans la même veine, un second point remet sur la sellette le lien quantitatif-statistiques. En effet, toujours selon Phillips et Burbules, l'un peut aller sans l'autre. Et un chercheur non positiviste peut très bien recourir aux méthodes statistiques et quantitatives. Cette nuance faite, laquelle est tout de même importante, nous continuerons l'élaboration de notre propos en statuant que, de façon générale, les bases des méthodes quantitatives et du paradigme positivisme se rejoignent et se complémentent dans leurs finalités.

En alternative au positivisme, plusieurs autres paradigmes existent. Se démarquant d'un positivisme trop rigide et univisionnaire, on ne pourra pas bien les lier, sinon pas du tout dans certains cas, à des méthodologies orientées vers l'approche quantitative. Dans la plupart des cas, pour ces autres paradigmes, l'approche qualitative sera proposée. D'ailleurs, Guba (1990) propose que l'inclusion accrue (mais non exclusive) de méthodes qualitatives dans le postpositivisme s'explique par la volonté de contrer une des limites du positivisme, soit le rapport débalancé entre la précision et la richesse des données recueillies. En effet, soutenues par des théories « interprétatives herméneutiques » ou 
" prescriptives », les recherches qualitatives utiliseront des démarches « inductives exploratoires ", " évaluatives fonctionnelles » ou axées sur la " conceptualisation spéculation », dans le but de recueillir des données « non métriques » aux fins d'analyse (Van der Maren, 1996). Ici, la recherche du sens, de la signification prévaut sur la corroboration ou l'infirmation. C'est d'ailleurs ce qu'expliquent Borg, Borg et Gall (1989) à propos de la recherche qualitative et de ses objectifs:

Les chercheurs qualitatifs [...] sont davantage amenés à étudier le cas individuel. Chaque individu, chaque école, chaque culture est susceptible de posséder ses propres systèmes de valeurs, de sentiments et de croyances qui ne peuvent être découverts qu'à travers des études approfondies et interactives de cet individu, cette école et cette culture. ${ }^{9}$ (p. 24 , traduction libre)

Savoie-Zajc (2003), dans un écrit publié dans le Dictionnaire des méthodes qualitatives en sciences humaines traitant de la pédagogie et des méthodes qualitatives, explique que:

Ce type de recherche repose sur une vision ontologique où la réalité se comprend de façon non plus morcelée mais globale et contextualisée. Le rapport au savoir est aussi différent dans la mesure où le sens produit est vu comme émergent et construit au cours de la recherche, fruit de l'interaction entre les participants et le chercheur. (p. 183)

Mucchielli, dans le même ouvrage, traite de la méthode qualitative en ces termes:

Une méthode qualitative est une succession d'opérations et de manipulations techniques [par exemple, transcriptions, découpages de texte, mises en tableau] et intellectuelles [par exemple: transpositions de termes en d'autres termes, regroupements intuitifs, confrontations à des savoirs, inductions généralisantes] qu'un chercheur fait subir à un objet ou un phénomène humain pour en faire surgir les significations pour luimême et les autres hommes. (p. 212-213)

Toujours dans ce dictionnaire, Paillé (2003) écrit sur la recherche qualitative et ce qui la caractérise. Il présente un tableau comparatif de la recherche quantitative et de la

\footnotetext{
9 "Qualitative researchers, however, are more likely to study the individual case. Each individual, each school, each culture is likely to have an idiosyncratic set of values, feelings, and beliefs that can only be discovered through intensive, interactive study of that individual, school, and culture. "
} 
recherche qualitative que nous reproduisons, celui-ci résumant très bien et de façon explicite les éléments propres à ces deux approches. En effet, en regard de plusieurs concepts jugés importants par Paillé (par exemple, mots-clés, approche, préoccupations, contexte, etc.), celui-ci présente ce qui est propre à la recherche qualitative et à la recherche quantitative. Le tableau met donc en contraste des éléments qui permettent de comprendre rapidement les bases de ces deux approches de recherche.

\section{Tableau 1}

Comparaison des pôles de la recherche quantitative et qualitative (Paillé, 2003)

\begin{tabular}{l|l}
\hline \multicolumn{1}{c|}{ Recherche quantitative } & \multicolumn{1}{c}{ Recherche qualitative } \\
\hline - Mots clés: Contrôle, Étendue & $\begin{array}{l}\text { - Mots-clés: Compréhension, Profondeur } \\
\text { - Approche ethnologique et de la } \\
\text { communication }\end{array}$ \\
- Préoccupée par: objectivité, & - Ces questions sont souvent secondaires \\
généralisabilité, reproductibilité & - Logique de la découverte \\
- Logique de la vérification & - A priori, tout peut être significatif \\
- On sait assez précisément ce qui sera & \\
significatif & - Contexte appréhendé \\
- Contexte posé & - Contrôle des « variables » a posteriori \\
- Contrôle des variables a priori & - Intérêt pour la « causalité » locale \\
- Peut établir des relations « causales » et & circulaire et symbolique \\
des corrélations & Procédures variables \\
- Procédures codifiées et fixes & - Compréhension et présentation de la \\
- Sublimation (ou négation, selon le & complexité \\
point de vue) de la complexité & - Les données sont considérées comme \\
- Les données sont considérées comme \\
étant « discrètes »
\end{tabular}

Source: Paillé, Dictionnaire des méthodes qualitatives en sciences humaines, p. 228.

Un dernier constat concernant l'approche qualitative nous amène à prendre conscience de l'utilisation de plus en plus présente des méthodes qualitatives dans les sciences humaines, ce qui inclut l'éducation. Pour répondre à la multitude des besoins disciplinaires et des finalités recherchées, il existe une panoplie de recherches dites à méthodologie qualitative disponibles au chercheur. Uniquement dans Recherche sociale De la problématique à la collecte de données (Gauthier, 2003), on élabore sur l'étude de 
cas, l'observation directe, l'entrevue semi-dirigée, l'histoire de vie, le groupe de discussion, l'analyse de contenu et la recherche-action. Cette liste n'est bien sûr pas exhaustive. Il y en existe en effet bien d'autres, ce type de recherche étant en effervescence.

L'importance de la compréhension des familles méthodologiques s'avère majeure, car c'est d'elles qu'émergent les procédures de recherche, en particulier la *méthode de recherche, laquelle doit être pertinente et éprouvée. Qu'est-ce qu'une méthode de recherche? Le tableau 2 reprend ce que propose Van der Maren (1996) à ce sujet:

Tableau 2

Définition d'une méthode recherche (Van der Maren, 1996)

Une méthode de recherche est

un ensemble d'opérations systématiquement et rationnellement enchaînées

afin de

- relier avec consistance

- l'intention, le but, l'objectif de la recherche

- la manière de poser le problème

- les techniques de constitution du matériel et de leur validation

- les techniques de traitement transformant les données en résultats

- les procédures d'interprétation des résultats et de leur

vérification

- la justification des différents choix

- répondre aux critères formels et opérationnels auxquels elles doivent s'astreindre pour se voir accorder la crédibilité recherchée.

Source: Van der Maren, Méthodes de recherche pour l'éducation, p. 112.

Ainsi, une méthode de recherche est complexe et complète en soi, devant répondre à plusieurs critères et ayant recours plusieurs types de techniques selon les étapes. Par exemple, l'observation n'est pas en soi une méthode de recherche, mais plutôt une méthode de cueillette de données, lesquelles devront ensuite être analysées, le tout à l'intérieur, par exemple, d'une étude phénoménologique. Il s'agit d'une clarification à faire, cet aspect méthodologique étant parfois nébuleux, ou abordé de façon erronée. Dans la section suivante, nous continuerons à traiter des méthodes de recherche, mais nous aborderons une famille spécifique de méthodes, soit les stratégies d'analyse secondaire. 


\subsection{Les stratégies d'analyse secondaire de recherches}

Alors que nous venons de faire un survol des bases de la recherche scientifique, nous avons pu remarquer qu'une conception largement acceptée de la recherche est la création de nouvelles connaissances à l'aide de nouvelles données. Nous continuons notre exercice en bifurquant vers un secteur de la recherche qui est parfois laissé pour compte. Il s'agit des stratégies d'analyse secondaire. Pour contextualiser, il faut reconnaître l'émergence d'un phénomène peu banal, soit l'accumulation d'études primaires publiées. Il y a dès lors, et de plus en plus, présence d'un corpus de connaissances scientifiques disponibles pour de nouvelles analyses. Cela permet de tirer profit des résultats de recherches et d'accéder à un large éventail de données, comme si le nombre de sujets interrogés était démesurément supérieur à la quantité de cas qu'il aurait été possible d'inclure dans une recherche primaire. En d'autres termes, les chercheurs ont accès à une mine d'or d'information. Nous aborderons la réutilisation de recherches scientifiques sous deux angles, soit l'analyse secondaire des données et l'analyse secondaire des résultats.

En effectuant des lectures dans le but de sélectionner une méthode de recherche pour effectuer une étude ou simplement en lisant sur la méthodologie de la recherche, un chercheur se rend vite compte qu'il existe deux grandes familles de méthodes de recherche, soit celles qui utilisent des données primaires (méthodes très répandues) et celles qui utilisent des*données secondaires (méthodes moins répandues). Si certains étaient tentés de prétendre que l'analyse secondaire est une forme de recherche moins " scientifique ", Turgeon et Bernatchez (2003) écrivent qu'il s'agit en fait d'une « alternative moins coûteuse, moins exigeante, plus rapide et parfois plus rigoureuse », que " dans un esprit très écologique, on peut "récupérer" des données dont on n'a pas extrait toute la valeur scientifique », que « les mêmes données [primaires] peuvent cependant livrer bien d'autres messages » (p. 431-432).

Turgeon et Bernatchez (2003) définissent les données secondaires comme des " éléments informatifs rassemblés pour des fins autres que celles pour lesquelles les 
données avaient été recueillies initialement "(p. 432). Pour traiter ces données, les chercheurs auront recours à *l'analyse secondaire, laquelle est « réalisée spécifiquement sur les données (secondaires) que vous exploitez aux fins de la nouvelle recherche » (Ibid.). Définie ainsi, l'analyse secondaire s'avère une approche très large et flexible. Pour imager de façon très simple un style d'analyse secondaire, il peut s'agir de reprendre un sondage fait à très grande échelle dans le cadre d'une étude $\mathrm{X}$, de porter son attention sur certaines questions spécifiques de ce sondage, les combiner (si désiré) à d'autres sondages comportant des questions similaires, et en faire ressortir des résultats nouveaux, résultats différents des buts visés par les premières études pour lesquelles ces sondages furent d'abord réalisés. Ici, ce qu'il faut comprendre, c'est que l'analyse secondaire des données apporte de nouveaux résultats, mais pas nécessairement une compréhension nouvelle du phénomène analysé a priori.

Si ce genre d'analyse secondaire est pertinent selon les arguments présentés ici, elle n'est pas acceptée de façon automatique par la communauté scientifique. Par exemple, dans le formulaire de demande d'évaluation éthique de la Faculté d'éducation de l'Université de Sherbrooke ${ }^{10}$, le chercheur doit préalablement à sa recherche indiquer si les données recueillies dans le cadre de la recherche anticipée seront réutilisées lors d'analyses secondaires pour des études comportant de nouveaux objectifs de recherche. Le chercheur devant cocher « oui » ou « non », s'il opte pour la négative, les données obtenues ne pourront servir aux fins proposées par Turgeon et Bernatchez.

Réutiliser des données pour atteindre de nouveaux objectifs n'est cependant pas la seule avenue disponible au chercheur qui désire faire avancer la science en se basant sur du matériel existant. Un autre genre d'analyse secondaire consiste en effet à porter son attention sur les résultats d'études déjà réalisées et non sur les données. Dans ce cas-ci, on voudra souvent en arriver à une synthèse des résultats des différentes études, agglomérées

\footnotetext{
${ }^{10}$ Formulaire de demande d'évaluation éthique, Comité d'éthique de la recherche - Éducation et sciences sociales, Université de Sherbrooke. Consulté le 13 avril 2006.

http://www.usherbrooke.ca/education/recherche/ethique/CER-ESS\%20demande.doc
} 
et analysées à l'aide d'un processus rigoureux. En éducation, comme dans d'autres domaines, on utilise souvent la méta-analyse pour faire ce travail. Originalement issue de l'approche quantitative, la méta-analyse a cependant son équivalent pour les études à données qualitatives. Mais à cause de ses inspirations quantitatives, la méta-analyse qualitative reste avec des visées de synthèse prépondérantes. Ces visées, tout de même non négligeables, satisferont les chercheurs qui désirent obtenir une vision élargie et souvent plus solide d'un phénomène donné. Ainsi, selon Zhao (1991), la méta-analyse de données (" meta-data-analysis ») comporte trois objectifs, trois composantes qui la caractérisent:

1. l'étude des suppositions qui sous-tendent les différentes procédures méta-analytiques;

2. la comparaison des différents types de données selon leur qualité et leur pertinence;

3. la synthèse des résultats recueillis à l'intérieur d'études portant sur un même phénomène. ${ }^{11}$ (p. 379, traduction libre)

Les buts d'une méta-analyse sont donc assez clairs: ils portent presque exclusivement sur les résultats et l'analyse même des données. Cependant, les métaanalyses ont leurs limites, du moins dans l'optique de Glass (1976). En effet, selon ce que présente Zhao (1991), l'approche proposée par Glass s'attarde principalement, sinon uniquement, sur le principe de la synthèse des résultats. En effet, Zhao cite Glass qui stipule qu'une méta-analyse consiste en « une analyse statistique d'un ensemble de résultats provenant d'analyses de différentes études dans le but d'intégrer ces résultats. ${ }^{12}$ (p. 385 , traduction libre) A priori, cette définition semble pertinente, sauf que par son élément de statistiques, elle met de côté du même coup presque tout le pan qualitatif de la recherche. C'est d'ailleurs pour cette raison que Noblit et Hare ont élaboré en 1988 une procédure s'inspirant de celle de Glass pour les données qualitatives. Mais, plus importante encore, l'avenue prise par Glass ne prône pas une réflexion sur le processus d'analyse des données. C'est d'ailleurs une des principales lacunes de la compréhension (et conséquemment de

\footnotetext{
11 * Meta-data-analysis consists of: (1) the study of the underlying assumptions of various data-analytic procedures; (2) the comparison of different forms of data in terms of their quality and utility; and (3) the synthesis of the findings of a range of research studies that are related to the same phenomenon."

12 "Meta-data-analysis refers to "the statistical analysis of a large collection of analysis results from individual studies for the purpose of integrating the findings" (Glass, 1976, p. 3). "
} 
l'utilisation) de la méta-analyse telle qu'on la pratique souvent de nos jours. En effet, il n'y a qu'à prendre la définition qu'en donne Legendre (2005) pour s'en rendre compte: "Analyse de contenu d'un grand nombre de conclusions d'études ou de recherches dans un domaine dans le but d'effectuer une synthèse de l'ensemble des résultats. " (p. 870) L'auteur propose également, comme concept similaire à la méta-analyse, la revue de la littérature. Dans cette optique, nous sommes à même de constater que malgré la pertinence d'effectuer des méta-analyses, celles-ci répondent à des besoins bien particuliers et sont limitées au regard des finalités à atteindre, que ce soit en quantitatif ou en qualitatif. Nous reviendrons sur les particularités de la méta-analyse qualitative ultérieurement.

Ce qu'il faut savoir, c'est qu'il est possible d'aller plus loin en analyse secondaire des résultats. Pour ce faire, une des options est d'avoir recours à une métaétude. Dans sa logique plus fondamentale et surtout plus complète que la méta-analyse, l'utilisation de la métaétude rend possible l'élaboration de nouvelles théories, objectif difficilement atteignable avec les méta-analyses. D'ailleurs, la méta-analyse est une des composantes de la métaétude. Les finalités qui lui sont propres ne représentent donc qu'une portion de l'ensemble des finalités possibles issues de l'emploi complet et complexe d'une métaétude. Pour comprendre cet aspect, prenons un instant pour porter un regard sur les métaétudes. Pour expliquer un peu plus ce qu'elles sont, nous nous inspirerons d'un article intitulé Metatheory, metamethod, meta-data-analysis: what, why, and how? (Zhao, 1991).

Zhao explique dans un premier temps que, comme le nom l'indique, les métaétudes font suite à d'autres études et ont pour visée d'étudier les résultats d'études et les procédés qui les ont générées. En bref, l'étude d'études. Cet objectif de recherche est bien sûr très différent de ce que nous sommes habitués de rencontrer. En effet, comme nous l'avons exprimé en début de section, l'analyse des données de la plupart des recherches scientifiques effectuées est normalement réalisée sur des données primaires. Ce n'est pas le cas des métaétudes, dont le but est de produire des analyses secondaires de résultats, reprenant des études déjà réalisées. Cependant, les métaétudes, dans l'idéal de la définition que propose Zhao, ne font pas que synthétiser ce qui a déjà été fait, ce qui est 
caractéristique des méta-analyses actuelles. Elles cherchent également à porter un regard interrogatif sur tout ce qui a permis d'en arriver aux résultats. En fait, le but est généralement de mieux comprendre un phénomène en extrayant des données recueillies une compréhension nouvelle, une vision conceptuelle innovatrice, une interprétation de second niveau. Pour ce faire, les métaétudes évalueraient les théories qui soutiennent les recherches étudiées, les méthodologies de celles-ci, de même que l'analyse des données et les résultats qui en découlent.

La " nouvelle » méthode de recherche que nous nous proposons d'étudier, la métasynthèse, rejoint davantage les visées des métaétudes telles que décrites par Zhao, avec toute la complexité qui les caractérise. Elle ne cherche pas qu'à synthétiser, mais plutôt à faire ressortir une nouvelle réalité découlant d'études qualitatives déjà complétées. Malheureusement, cette forme d'étude est plutôt marginalisée au profit de la méta-analyse qualitative (dans sa forme axée sur la synthèse) et les deux termes sont parfois même confondus, interchangés. De par son intérêt, mais également à cause de l'ambiguïté qui l'entoure, clarifier le concept de la métasynthèse s'avère donc une entreprise pertinente à entreprendre.

\section{LA DESCRIPTION DU PROBLÈME}

La section précédente a présenté le contexte scientifique de la recherche en éducation. En effet, nous avons vu que la recherche en éducation est nécessaire, comme dans tous les domaines. Pour être fiable et pertinente, la recherche se doit d'être scientifique, suivant certaines démarches qui assureront rigueur et transparence. Par ailleurs, ce sont les paradigmes qui orientent la recherche scientifique et ses composantes. Ceux-ci ont une influence sur les choix méthodologiques de la recherche, lesquels se divisent essentiellement en deux grandes familles: l'approche quantitative et l'approche qualitative. C'est cette dernière qui nous intéresse, s'étant taillé une grande place en éducation. Nous avons également vu qu'il existe une sorte de recherche assez particulière: les métaétudes. Celles-ci, s'inscrivant dans les stratégies d'analyse secondaire, ont pour but 
de jeter un regard nouveau sur des études complétées ou encore de faire une synthèse de résultats d'études déjà effectuées, une orientation très populaire de nos jours. Cette visée des méta-analyses demeure pertinente, mais restreinte dans la portée réflexive qui existe originalement dans les caractéristiques propres aux métaétudes.

Or, voilà que nous sommes mis en connaissance d'une autre méthode de recherche et d'analyse secondaire d'études qualitatives, une métaétude nommée métasynthèse. Selon Banning, cette méthode offrirait un potentiel plus intéressant encore que la méta-analyse qualitative. Nous sommes donc particulièrement intéressé à étudier cette méthode pour connaître ses principes et la manière de l'utiliser.

\section{3. $\cdot$ LA FORMULATION DE LA QUESTION GÉNÉRALE DE RECHERCHE}

Compte tenu des aspects de la recherche scientifique en éducation qui viennent d'être présentés et des possibles apports bénéfiques d'une nouvelle méthode de recherche qu'est la métasynthèse, nous nous y intéressons et posons le questionnement suivant:

Quels sont les caractéristiques de la métasynthèse et les fondements de son utilisation et en quoi se distingue-t-elle de la méta-analyse qualitative?

\section{LA RECENSION DES ÉCRITS SUR LA MÉTASYNTHÈSE}

Dans le but de répondre à la question générale de recherche, nous avons entrepris une recension des écrits. Cette section présente cette recension, laquelle a été faite dans l'optique de trouver des écrits traitant de la métasynthèse comme méthode de recherche dans les sciences humaines, les sciences sociales et les sciences de l'éducation. Dans un premier temps, des livres ont été examinés. Ensuite, des banques de données permettant l'accès à un très large volume d'articles scientifiques ont été explorées. La section se termine avec l'état de la question. 


\subsection{Les ouvrages méthodologiques}

Pour tenter de répondre à la question générale de recherche, nous avons tout d'abord recensé plusieurs livres de référence sur la recherche en sciences humaines, sociales et de l'éducation. Il s'agissait de voir ce que les auteurs avaient écrit sur la métasynthèse.

Pour effectuer cette étape, nous avons fait une recherche d'informations en deux temps. Dans un premier temps, nous nous sommes basé sur les sections « Références bibliographiques » des plans de cours de différents séminaires offerts à la maîtrise et au doctorat en sciences de l'éducation de l'Université de Sherbrooke, automne-hiver 2005 $2006^{13}$. Nous avons choisi de passer en revue les textes de référence abordant la méthodologie de la recherche, les types de recherche, les procédures de recherche, orientés vers la recherche qualitative. Nous n'avons pas eu recours à des ouvrages où l'emphase est mise sur l'approche quantitative. Les livres sélectionnés et disponibles pour consultation ont été passés en revue. Avec surprise, les résultats sont les suivants: parmi les 24 ouvrages recensés ${ }^{14}$, aucun ne mentionne la métasynthèse comme une forme de recherche disponible pour des études en sciences humaines, en sciences sociales et en sciences de l'éducation.

Dans un deuxième temps, nous avons décidé d'élargir le nombre d'ouvrages explorés pour en trouver qui abordaient la métasynthèse. Nous avons ainsi sélectionné à la bibliothèque d'autres livres traitant de la recherche dans les sciences humaines, sociales et de l'éducation. Encore une fois, la recherche nous a forcé à constater l'absence de notre concept central dans les livres: parmi les 27 ouvrages recensés ${ }^{15}$, aucun ne mentionne la métasynthèse comme une forme de recherche disponible pour des études en sciences humaines, sociales et de l'éducation.

\footnotetext{
${ }^{13}$ Nous avons obtenu des plans de cours des quatre séminaires en éducation offert au campus principal et les deux offerts au campus de Longueil.

${ }^{14} \mathrm{La}$ liste de ces ouvrages se retrouve à l'Annexe A.

${ }^{15} \mathrm{La}$ liste de ces ouvrages se retrouve à l'Annexe B.
} 
Conséquemment, sur un total de 51 livres traitant de la recherche en sciences humaines, sociales et de l'éducation, aucun ne traite de la métasynthèse. Il s'agit d'une lacune flagrante dans la littérature méthodologique des domaines ci-haut mentionnés.

L'analyse de notre démarche et des constats auxquels nous sommes arrivé nous permet ici de faire quelques commentaires. Premièrement, il faut savoir que la quantité d'ouvrages récents en méthodologie de la recherche en sciences humaines, sociales et de l'éducation n'est pas extrêmement élevée, spécialement du côté de l'approche qualitative, comparativement à l'approche statistique, pour ne donner qu'un exemple. Deuxièmement, le fait qu'aucun des ouvrages recensés ne présente la métasynthèse démontre bien une lacune dans les connaissances sur les méthodes disponibles. Or, même si un ouvrage, qui n'aurait pas été consulté ici, en discutait, il n'en demeure pas moins que nous ne pourrions pas parler d'une forme de recherche bien explicitée et populaire. Troisièmement, c'est dans cette optique qu'il est bon de souligner que le but de notre recension dans ces domaines de recherche n'a pas été de consulter la totalité des livres méthodologiques en sciences humaines, sociales, et de l'éducation, mais de consulter ceux qui semblent les plus largement utilisés. Bien sûr, le fait de consulter le plus grand nombre de textes possible sur le sujet est l'idéal. Mais actuellement, nous avons plutôt établi une tendance: l'absence d'information concernant la métasynthèse dans les livres de méthodologie couramment utilisés.

Après avoir porté un regard sur les livres de méthodologie de la recherche, nous avons opté de continuer notre recension avec la recherche d'articles scientifiques à teneur méthodologique, toujours dans le domaine des sciences sociales, sciences humaines et sciences de l'éducation. 


\subsection{Les articles en sciences humaines, sociales et de l'éducation}

Puisque la recension de livres de méthodologie traitant de la métasynthèse, ou comportant un chapitre sur le sujet, s'est avérée infructueuse, l'étape suivante a consisté à fouiller les banques d'articles scientifiques dans le but de trouver des écrits traitant de ces formes de recherche.

Pour ce faire, les banques électroniques anglaises ERIC (Education Resources Information Center) par ProQuest furent tout d'abord utilisées. En ayant recours à une recherche du terme metasynthesis à l'intérieur de citation and abstract: deux textes sont référencés. Le premier concerne une étude ${ }^{16}$ en sciences infirmières qui a eu recours à la métasynthèse. Cet article n'appartient pas au type de textes recherchés dans le domaine des sciences humaines, sociales ou de l'éducation et il ne traite pas de la métasynthèse comme sujet de l'écrit, mais plutôt comme la méthode de recherche utilisée. Pour l'instant, nous désirons trouver des textes traitant de la métasynthèse. Le deuxième article ${ }^{17}$ relevé n'est pas relié directement à la métasynthèse. On y parle de méta-analyse et de synthèse, et l'étude est de nature quantitative, ce qui ne correspond pas à notre objet de recherche. Par ailleurs, puisque nous recherchons des publications traitant de la métasynthèse, et non pas particulièrement des articles dont la procédure de recherche est une métasynthèse, cet article, qui traite des enfants doués et du regroupement, ne correspond pas à nos critères de sélection. Cette recension faite à partir de la base de données ERIC, la principale dans le domaine de l'éducation, nous démontre pour ainsi dire l'inexistence de notre concept central dans ce champ d'intérêt. Élargissons maintenant notre procédure de recension vers d'autres domaines en sciences sociales.

\footnotetext{
${ }^{16}$ Beck, C.T. (2001). Caring within nursing education: a metasynthesis, Journal of Nursing Education, 40(3), 101-109.

${ }^{17}$ Allan, S.D. (1991). Ability-grouping research reviews: What do they say about grouping and the gifted? Educational Leadership, 48(6), 60-65.
} 
Ensuite, comme deuxième banque d'écrits, nous avons utilisé PsychARTICLES avec la même procédure, soit l'utilisation du mot metasynthesis dans citation and abstract. Cette fois, aucun document n'est référencé.

Enfin, nous avons combiné les modules Education, Humanities, Psychology et Social Science dans la ProQuest Research Library pour effectuer une recherche très large, avec les mêmes éléments de sélection (metasynthesis dans citation and abstract). La ProQuest Research Library regroupe une multitude de formes d'écrits comme l'indique sa description: Search the full collection of journals, magazines and newspapers for information on a broad range of general reference subjects. Cette fois, un texte est référencé, mais il s'agit du même que celui trouvé dans ERIC, en sciences infirmières.

\subsection{Les articles disponibles dans d'autres domaines}

Lors de notre consultation de la banque de données ERIC pour notre recension des écrits, nous avons recueilli deux articles avec le mot-clé «metasynthesis ». L'un d'eux est dans le domaine des sciences infirmières et s'intitule Caring within Nursing Education: A Metasynthesis (Beck, 2001). Cet article a la particularité de proposer comme méthode de recherche la métasynthèse. Or, pour qu'un chercheur ou une chercheuse puisse entreprendre une étude scientifique, il faut que la méthode employée soit reconnue et approuvée par la communauté scientifique dans son domaine. Dans cet esprit, il est apparu logique de chercher dans le domaine des sciences infirmières des textes sur la métasynthèse. C'est ce que nous avons fait, cela nous a permis de sélectionner neuf articles jugés pertinents à la compréhension de la métasynthèse. ${ }^{18}$ Voici comment nous avons procédé.

Tout d'abord, un moyen utilisé fut la banque de données PubMed. Celle-ci, disponible sur Internet, propose l'accès à plusieurs millions d'articles appartenant au large

\footnotetext{
${ }^{18}$ Les textes répertoriés sont présentés dans l'annexe C
} 
domaine des sciences biomédicales. Ensuite, un autre moyen utilisé pour trouver des textes pertinents à notre projet a été de se baser sur les références bibliographiques du texte de Banning (s.d.), lequel fut en quelque sorte le précurseur du présent travail. C'est d'ailleurs de cette liste que provient l'article de Zhao, utilisé lors de la présentation des types de méta-analyses et des métaétudes dans le premier chapitre. De cette liste de références provient également un ouvrage très important, Meta-study of qualitative health research : a practical guide to meta-analysis and meta-synthesis (Paterson et al., 2001). Ce livre fut lui aussi une source de références permettant de trouver des textes pertinents à notre recherche. Un dernier moyen utilisé fut la recherche d'informations sur Internet. Ainsi, à l'aide du terme « metasynthesis » ou encore avec le nom de certains auteurs ayant écrit des textes sur la métasynthèse, quelques textes furent trouvés, parfois les mêmes que ceux disponibles dans PubMed. En tout et partout, comme nous l'avons mentionné, ce sont finalement neuf articles et un livre qui ont ainsi été répertoriés. Comme nous voyons, la quantité de textes traitant de la métasynthèse n'est pas extrêmement élevée; néanmoins, il s'agit d'un bon corpus utile à sa compréhension. C'est pourquoi nous avons décidé de nous référer à ces textes en tant que matériel d'analyse dans le cadre du présent travail. Cela devrait nous permettre de répondre à l'objectif de notre recherche en concordance avec la méthodologie qui est proposée au chapitre trois.

\section{LA RECENSION DES ÉCRITS SUR LA MÉTA-ANALYSE QUALITATIVE}

Les livres de méthodologie de la recherche étant plus explicites au sujet de la métaanalyse qualitative, nous n'avons pas cherché à trouver une panoplie de textes la décrivant. Cependant, tout comme nous venons de l'expliquer pour la métasynthèse, nous jugions intéressant d'être en mesure de voir comment cette approche était utilisée de façon concrète. Dans cette optique, nous avons répertorié dans ERIC quatre études qui sont des méta-analyses qualitatives. ${ }^{19}$ Fait intéressant, un autre texte que nous avions trouvé s'est avéré, après lecture, avoir plutôt comme sujet la métasynthèse. Ainsi, malgré son titre,

\footnotetext{
${ }^{19}$ Les textes répertoriés sont présentés dans l'annexe D
} 
Reinterpretation across studies: an approach to meta-analysis (McCormick, Rodney, Varcoe, 2003), nous comptons l'utiliser dans la discussion de la métasynthèse.

\section{L'ÉTAT DE LA QUESTION}

Nous croyons qu'il est juste d'affirmer, à la suite de l'utilisation de trois banques de données et des résultats plutôt limités qui en ont découlé, que la méthode de la métasynthèse n'est pas encore répandue en sciences de l'éducation comme en sciences humaines et sociales. Cette affirmation est également supportée par l'absence du concept dans plusieurs ouvrages méthodologiques en sciences humaines, sciences sociales, et en sciences de l'éducation. Cependant, nous avons relevé un article en sciences infirmières référencé par ERIC et ProQuest dont l'auteure a utilisée la méthode de la métasynthèse. Cela nous a conduit à vérifier dans d'autres banques de données portant sur la recherche biomédicale la présence de recherches basées sur la métasynthèse. Nous en avons trouvé un certain nombre en sciences infirmières et nous croyons qu'il s'agit là d'une opportunité de mieux comprendre la métasynthèse et son utilisation, d'éventuellement pouvoir transférer des connaissances dans le domaine de sciences humaines, sociales et de l'éducation.

Cependant, pour clarifier la position de la métasynthèse dans le champ des stratégies d'analyse secondaire, nous voulons déterminer ce qui la caractérise par rapport à la méta-analyse qualitative. Pour ce faire, nous croyons opportun d'utiliser quelques recherches ayant eu recours à la méta-analyse qualitative et déterminer ce qui caractérise cette approche, de même que les similitudes et les différences entre ces deux méthodes. Nous avons répertorié quelques textes qui ont été utilisés à cette fin. 


\section{L'OBJECTIF DE LA RECHERCHE}

Étant donné le manque de connaissance en sciences humaines, sociales et de l'éducation concernant la métasynthèse et la plus grande popularité de la méta-analyse qualitative, le présent projet propose l'objectif suivant:

- Comparer la métasynthèse et la méta-analyse qualitative dans l'optique de les caractériser et de les distinguer.

Le prochain chapitre, soit le cadre conceptuel, se penche sur certains éléments de notre recherche qui doivent être approfondis et explicités pour bien démontrer la pertinence de notre travail et le positionnement de notre objet. 


\section{CHAPITRE DEUX \\ LE CADRE CONCEPTUEL}

Dans ce chapitre, trois éléments sont étudiés. Tout d'abord, nous abordons les similarités qui existent entre la recherche effectuée en sciences infirmières et celle pratiquée en éducation afin de rendre pertinent un transfert interdomaine d'une méthode recherche. Ensuite, nous présentons d'autres méthodes qui ont comme objectif de synthétiser des données qualitatives, dans le but de les situer et de voir les différences qui existent dans cette famille. De plus, cette partie sert de base à notre dernière partie. En effet, nous nous penchons alors sur les caractéristiques descriptives à utiliser dans le but d'atteindre notre objectif de recherche et en regard de la méthodologie qui est proposée au chapitre trois.

\section{LES SIMILITUDES ENTRE LES SCIENCES INFIRMIÈRES ET DE L'ÉDUCATION}

Nous avons vu dans le précédent chapitre qu'il n'existe aucune référence propre au domaine des sciences de l'éducation concernant la méthode de recherche qu'est la métasynthèse. Cependant, nous avons été en mesure de trouver des textes en sciences infirmières qui nous ont permis de répondre en partie à notre question générale de recherche. Notre volonté a consisté alors à aller chercher dans ce domaine des informations sur la métasynthèse pour ensuite proposer cette méthode en éducation. À première vue, l'idée semble valable. Cependant, pour ce faire, nous devons nous pencher sur une question plus fondamentale, à savoir dans quelle mesure le transfert de l'applicabilité d'une méthode de recherche du domaine des sciences infirmières vers le domaine des sciences de l'éducation est cohérent et valable. En effet, dans quelle mesure une forme de recherche utilisée en soins infirmiers peut-elle se révéler à propos en éducation? C'est ce que nous allons examiner dans la présente section. 
Pour déterminer si nous pouvions légitimement transposer en éducation une méthode de recherche surtout présente en sciences infirmières, notre intention de départ consistait à démontrer qu'il existait suffisamment de similarités entre ces deux domaines, spécialement au regard des méthodes de recherche utilisées, pour justifier notre démarche. Nous voulions ainsi recenser des méthodes propres à chacun des domaines et ensuite les comparer. Cela semble simple, mais il ne s'agit pas d'une opération aussi aisée qu'il n'y paraît. En effet, la littérature méthodologique concernant l'éducation et les sciences infirmières n'est pas toujours spécifique à chacun des domaines. Par exemple, il n'y a pas réellement d'ouvrages intitulés « La mise en oeuvre concrète des diverses méthodes de recherche en éducation ». Nous retrouvons davantage de livres orientés vers le grand champ des sciences sociales. Nous nous sommes donc questionné sur ce fait en nous penchant davantage sur le concept des sciences sociales et les stratégies de recherches qui les soutiennent.

C'est ainsi que nous avons examiné le livre Social research methods : qualitative and quantitative approaches de Bernard (2000). L'auteur y définit les *sciences sociales d'une manière très englobante: « les sciences de la pensée et du comportement humain. » (p. 4, traduction libre $)^{20}$ Aussi, selon Legendre (2005), les sciences sociales sont une " locution désignant le domaine du savoir occupé par la pensée et l'agir humain dans leur dimension collective plus qu'individuelle et plus précisément par le comportement humain en général. » (p. 1218) Là où il semble y avoir ambiguitté, c'est lorsque vient le temps de différencier les sciences sociales et les sciences humaines, champ dans lequel est souvent située l'éducation. En nous référant toujours à Legendre (2005), nous constatons plusieurs éléments: premièrement, l'expression anglaise qui traduit " sciences humaines .» est " social sciences »; deuxièmement, les sciences humaines seraient le « domaine du savoir correspondant à ce que l'anglais appelle de façon moins ambitieuse "social sciences" ” (Ibid.); et troisièmement, sous sciences sociales, Legendre note que « le groupe de sciences particulières ici concerné recouvre presque exactement le domaine proprement français des

\footnotetext{
20 " the sciences of human thought and human behavior."
} 
" sciences humaines », lequel correspondant pour l'essentiel aux "social sciences" des anglo-saxons. " (Ibid.). Le constat à faire ici est le suivant: les concepts de sciences sociales, sciences humaines et social sciences sont très larges dans leurs définitions, très similaires, voire interchangeables, mais surtout ils posent problème dans la mesure où leurs limites ne sont pas toujours précisées et qu'il n'existe pas de consensus général dans la littérature pour les définir. D'ailleurs, Dubouchet (1997) dira des sciences sociales qu'« elles étudient l'homme en société. C'est pourquoi elles se confondent à la fois avec les sciences sociologiques au sens large et avec les sciences humaines ou sciences de l'homme. » (p. 5)

Toujours est-il que les éléments les plus intéressants et les plus pertinents pour nos besoins concernant les sciences sociales nous proviennent du livre de Bernard (2000). Tout d'abord, Bernard inclut dans les sciences sociales non seulement les sciences de l'éducation, mais aussi les sciences infirmières. En effet, selon cet auteur, elles font toutes deux parties de la même famille, selon la définition que nous avons relevée plus haut. Plus spécifiquement, si ces deux sciences ne font pas partie des principales branches généralement attribuées aux sciences sociales, Bernard les qualifie de disciplines dans lesquelles est pratiquée la recherche sociale:

Le paysage des sciences sociales est particulièrement compliqué. Les principales branches, en ordre alphabétique, sont l'anthropologie, l'économie, l'histoire, les sciences politiques, la psychologie, la psychologie sociale et la sociologie. Chacun de ces champs possède des sous-champs et il y a, en plus, plusieurs disciplines dans lesquelles est pratiquée la recherche sociale. Celles-ci incluent les communications, la criminologie, la démographie, l'éducation, le journalisme, l'étude des loisirs, les soins infirmiers et le travail social, pour n'en nommer que quelques-unes. (p. 6, traduction libre) ${ }^{21}$

\footnotetext{
21 * The social science landscape is pretty complicated. The main branches, in alphabetical order, are anthropology, economics, history, political science, psychology, social psychology, and sociology. Each of these fields has many subfields, and there are, in addition, many other disciplines in which social research is done. These include communications, criminology, demography, education, journalism, leisure studies, nursing, and social work, to name just a few. "
} 
Plus intéressant encore sur le plan de la méthodologie de la recherche est le fait que dû à leur proximité, tous les domaines faisant parti des sciences sociales ont des buts similaires, bien que propres à leurs objets d'étude, et les façons d'atteindre les objectifs de recherche ont également plusieurs points en commun. Ainsi, le point le plus important est le constat suivant: ce sont souvent les mêmes méthodes de recherche qui sont employées dans les différents domaines des sciences sociales. Selon Bernard: « Avec le temps, des méthodes pour effectuer de la recherche ont été développées à l'intérieur de chacun des champs [des sciences sociales], mais aucune discipline n'a de droit de possession sur une méthode. " (Ibid., traduction libre ${ }^{22}$ Il est donc tout à fait pertinent d'utiliser en sciences de l'éducation une méthode qui sera utilisée également en sciences infirmières. Pour supporter cet argument, il convient de jeter un regard sur certaines caractéristiques de la recherche pratiquée en sciences infïmières et en sciences de l'éducation. Tout d'abord, la recherche en sciences infirmières se divise essentiellement en deux branches: une plus axée vers l'aspect expérimental, biologique; la seconde axée plutôt vers la pratique clinique, plus proche des sciences humaines. Dans la seconde approche, une partie de la recherche s'attardera à étudier la pratique infirmière dans l'optique de l'améliorer. Or, cela rejoint certains buts de la recherche en éducation, tel que démontré à la section 1.1 du premier chapitre. Mais plus encore, la recherche en soins infirmiers sert de multiples objectifs supplémentaires, comme l'administration ou encore l'enseignement en sciences infirmières. Cet aspect multipliscinaire est bien sûr partagé par les visées de la recherche en sciences de l'éducation, ce qui rapprochent ces deux domaines encore une fois. (Burns et Grove, 2005)

Comme dernier argument, il est intéressant de noter que certains livres de méthodologie de la recherche originalement dédiés à un domaine sont parfois repris dans d'autres. Un bon exemple est l'ouvrage Le processus de la recherche: de la conception à la réalisation, écrit sous la direction de Marie-Fabienne Fortin, professeure à la Faculté des sciences infirmières de l'Université de Montréal. En effet, celui-ci est parfois utilisé dans divers cours et séminaires au contenu méthodologique en sciences de l'éducation, les

\footnotetext{
22 "Over time, methods for research have been developed within each of these fields, but no discipline owns any methods. "
} 
différentes caractéristiques de la recherche dans ces deux disciplines étant partagées. Ce point, ajouté à ceux présentés dans les paragraphes précédents, valide à nos yeux la transférabilité de la métasynthèse des sciences infirmières aux sciences de l'éducation.

\section{LES MÉTHODES AYANT POUR OBJECTIF LA SYNTHĖSE QUALITATIVE}

La métasynthèse n'est pas une méthode de recherche tout à fait nouvelle, tout comme son genre n'est pas entièrement nouveau également. Le but ultime étant de permettre une synthèse de données qualitatives (à divers niveaux), d'autres types de recherches font partie de cette famille. Dans cette section, nous nous basons sur un article, Synthesising qualitative and quantitative evidence: a review of possible methods (DixonWoods, Agarwal, Jones, Young, Sutton, 2005), pour présenter d'autres façons de synthétiser des données, essentiellement qualitatives, chacune de ces approches ayant ses particularités, ce qui les rend distinctes, différentes de la métasynthèse. Nous remarquerons que la métaétude, méthode que nous avons abordé précédemment, est par ailleurs incluse dans les approches traités par cet écrit.

D'entrée de jeu, les auteurs discutent des besoins de méthodes de recherches dont l'objectif consiste à agglomérer, à réinterpréter, à synthétiser des données primaires d'études complétées. Ils expriment sous deux points le besoin de se pencher sur les méthodes existantes permettant de traiter les données qualitatives: le premier réside dans le constat que ce type de méthode a connu une certaine popularité ces dernières années, mais essentiellement sur le plan quantitatif (versus qualitatif); le deuxième point consiste en un élément d'efficacité, à savoir le besoin de se mettre au fait des méthodes de synthèse déjà existantes pour éviter d'en inventer de nouvelles inutilement. Peaufiner les méthodes existantes sera très souvent suffisant et pertinent. Ce dernier aspect, soit l'amélioration des méthodes actuelles, résulte d'ailleurs d'un constat général fait par les auteurs, se rendant compte que la plupart des méthodes étudiées ne sont pas toutes clairement définies dans leurs finalités, leurs modalités, etc. Ils terminent d'ailleurs leur texte en exprimant un 
« besoin urgent pour des méthodes rigoureuses pour synthétiser les diverses formes de résultats générées par les diverses méthodologies. » (p. 52, traduction libre $)^{23}$

Les auteurs de cet article ont recensé 11 méthodes « cousines » de la métasynthèse. Certaines sont dérivées de l'analyse de données primaires, d'autres ont été façonnées pour répondre à un besoin de synthèse de données qualitatives ou encore quantitatives. Voyonsles de façon succincte à l'aide d'un tableau résumant les caractéristiques principales de chacune d'elles.

Tableau 3

Présentation sommaire de méthodes d'analyse secondaire qualitatives

\begin{tabular}{|c|c|}
\hline MÉTHODES & DESCRIPTION \\
\hline Le résumé narratif & $\begin{array}{l}\text { Il s'agit de décrire et d'ordonner des éléments narratifs, avec } \\
\text { commentaires et interprétations de la part du chercheur. Sans } \\
\text { procédures bien établies, cette technique peut être critiquée du fait } \\
\text { de sa trop grande dépendance de la vision ou des connaissances du } \\
\text { chercheur, mais peut utiliser un très large éventail de types de } \\
\text { données, simultanément quantitatives ou qualitatives. Le résumé } \\
\text { narratif consiste donc à amasser des données de tous genres } \\
\text { relatives à l'objet de recherche et peut aboutir à une théorie. }\end{array}$ \\
\hline $\begin{array}{l}\text { L'analyse } \\
\text { thématique }\end{array}$ & $\begin{array}{l}\text { A l'intérieur d'une revue de la littérature aux orientations portées } \\
\text { vers un objet de recherche spécifique, il s'agit d'identifier des } \\
\text { thèmes importants et/ou récurrents. Une fois ces thèmes déterminés, } \\
\text { on répertorie sous chacun d'eux les éléments-résumés décelés dans } \\
\text { chacun des textes étudiés. Malgré que son aspect plutôt descriptif } \\
\text { peut s'avérer un problème en ce sens, il est possible de théoriser. } \\
\text { Cependant, le choix des thèmes est plutôt subjectif, ou du moins le } \\
\text { processus de sélection n'est pas nécessairement rigoureux et clair. } \\
\text { En retour, cela donne au chercheur une plus grande flexibilité. }\end{array}$ \\
\hline La théorie ancrée & $\begin{array}{l}\text { Essentiellement, la théorie ancrée consiste à comparer des données } \\
\text { primaires pour y déceler des similarités et des interrelations à } \\
\text { l'intérieur d'un échantillon jusqu'à l'obtention d'une saturation } \\
\text { théorique. Il existe plusieurs variantes de la théorie ancrée, ce qui } \\
\text { rend plus compliquée une compréhension claire et précise, tout } \\
\text { comme son procédé n'est pas toujours transparent. Le concept de la } \\
\text { saturation théorique a cependant comme avantage de limiter le }\end{array}$ \\
\hline
\end{tabular}

\footnotetext{
${ }^{23}$ "There is an urgent need for rigourous methods for synthesising evidence of diverse types generated by diverse methodologies. "
} 


\begin{tabular}{|c|c|}
\hline & $\begin{array}{l}\text { nombre d'études à inclure dans la recherche, le type d'étude pouvant } \\
\text { d'ailleurs être varié. }\end{array}$ \\
\hline $\begin{array}{l}\text { La } \\
\text { métaethnographie }\end{array}$ & $\begin{array}{l}\text { A l'aide d'une analyse de transposition réciproque (reciprocal } \\
\text { translational analysis), on détermine les thèmes clés de chacune } \\
\text { des études prises en compte, pour ensuite essayer de transposer ces } \\
\text { thèmes dans les autres études utilisées. Les thèmes les plus } \\
\text { englobants seront sélectionnés. Parfois limitée (par choix) à des } \\
\text { études primaires issues d'un seul paradigme, il peut en résulter des } \\
\text { théories généralisantes, d'un niveau supérieur. }\end{array}$ \\
\hline $\begin{array}{l}\text { La synthèse } \\
\text { réaliste }\end{array}$ & $\begin{array}{l}\text { Servant surtout à tester des théories, cette méthode implique le } \\
\text { choix d'un échantillon d'études précis à analyser, en gardant le } \\
\text { contexte des données. Le choix des études peut cependant causer } \\
\text { problème, celles-ci étant généralement prises clairement dans } \\
\text { l'optique de répondre aux objectifs visés. }\end{array}$ \\
\hline $\begin{array}{l}\text { La méthode de } \\
\text { Miles et } \\
\text { Huberman }\end{array}$ & $\begin{array}{l}\text { L'objectif visé est de déceler les similitudes et différences dans les } \\
\text { études primaires analysées. Pour ce faire, on résume et code les } \\
\text { données de chacune des études sous plusieurs thèmes, pour ensuite } \\
\text { les combiner. Il n'y a pas réellement de façon formelle de } \\
\text { sélectionner les études utilisées, mais le processus d'analyse a } \\
\text { l'avantage d'être rigoureux, de pouvoir inclure divers types de } \\
\text { données et de potentiellement mener à l'élaboration de théories. }\end{array}$ \\
\hline $\begin{array}{l}\text { L'analyse de } \\
\text { contenu }\end{array}$ & $\begin{array}{l}\text { Dans sa forme quantitative, il s'agit de coder un texte à l'aide de } \\
\text { thème pour ensuite compter les récurrences. Une variante consiste } \\
\text { plutôt à interpréter les thèmes ressortis. Il est possible d'utiliser } \\
\text { cette méthode dans un but de théorisation, si son objectif est autre } \\
\text { que de simplement décrire. Cependant, si l'emphase est mise sur la } \\
\text { fréquence d'apparition des thèmes, cela peut se faire au détriment } \\
\text { de l'importance, du contexte et de l'interprétation des données. }\end{array}$ \\
\hline $\begin{array}{l}\text { L'étude de cas } \\
\text { (case survey) }\end{array}$ & $\begin{array}{l}\text { Après avoir analysé chaque étude comme un cas particulier, mais à } \\
\text { l'aide de questions prédéterminées, on utilise des méthodes propres } \\
\text { au sondage sur les résultats obtenus. Cela permet d'étudier les } \\
\text { résultats, à défaut de pouvoir le faire sur les processus, ayant du } \\
\text { moins l'avantage de pouvoir analyser un grand nombre d'études } \\
\text { primaires, et possiblement résulter en l'élaboration d'une théorie. }\end{array}$ \\
\hline $\begin{array}{l}\text { L'analyse } \\
\text { qualitative } \\
\text { comparative }\end{array}$ & $\begin{array}{l}\text { Servant essentiellement à déterminer des principes de causalité, } \\
\text { versus l'interprétation de données qualitatives, il s'agit de s'appuyer } \\
\text { sur la présence ou l'absence de variables indépendantes et de } \\
\text { résultants dans chacune des études primaires. L'analyse booléenne } \\
\text { sera utilisée, ce qui en fera une méthode transparente, systématique. }\end{array}$ \\
\hline $\begin{array}{l}\text { La méta-analyse } \\
\text { bayesienne }\end{array}$ & $\begin{array}{l}\text { S'inscrivant dans le paradigme de Bayes, il s'agit de démontrer les } \\
\text { effets de la combinaison de variables issues de recherches } \\
\text { qualitatives, lesquelles sont supportées par des résultats d'études } \\
\text { quantitatives. Bien que l'idée semble simple, son application } \\
\text { pourrait se montrer techniquement ardue, mais le résultat pourrait }\end{array}$ \\
\hline
\end{tabular}




\begin{tabular}{l|l}
\hline & être l'élaboration d'une théorie. \\
\hline & $\begin{array}{l}\text { Elaborées selon un cadre précis (question de recherche, objectifs, } \\
\text { processus de sélection des études analysées, etc.), les métaétudes }\end{array}$ \\
Lent divisées en grandes parties ayant chacune un rôle précis à \\
accomplir (métathéorie, métaméthode, méta-analyse). Cette \\
méthode permet de voir les différents objectifs et résultats possibles \\
de la synthèse d'études, cependant strictement liées à l'approche \\
qualitative.
\end{tabular}

\section{$2.1 \quad$ Un exemple en éducation}

Les sciences de l'éducation, comme tous les domaines, ont développé avec les années un important corpus d'études. Plus récemment, l'approche qualitative a gagné en popularité, ce qui a mené à la production de nombreuses études dont les objectifs consistaient à explorer, décrire, décortiquer différents phénomènes liés à d'innombrables aspects présents en éducation. Éventuellement, une synthèse rigoureuse d'études allait s'avérer pertinente, et c'est l'exercice qu'ont proposé Gauthier, Martineau, Malo, Desbiens et Simard dans Pour une théorie de la pédagogie (1997). Ainsi, 42 textes furent retenus par l'équipe dirigée par Gauthier et c'est à l'aide de la méthode de Miles et Huberman qu'ils furent analysés. C'est donc sur une multitude de facettes, composantes de la pédagogie (par exemple, la gestion de la matière et la gestion de la classe), qui avaient déjà été étudiées, mais que Gauthier et son équipe ont réanalysées, qu'ils en ont fait ressortir un sens nouveau, plus complet, plus complexe et plus englobant. La pertinence d'un tel type d'étude est très bien exposée dans cet extrait tiré de l'avant-propos du livre :

S'appuyant sur les nombreuses recherches menées sur ce problème [les connaissances liées aux savoirs professionnels des enseignants], ces auteurs [d'études] affirment qu'il existe désormais une base de connaissances pédagogiques qui permet aux enseignants de mieux enseigner aux étudiants. Selon plusieurs, les nombreuses recherches sur l'enseignement conduites ces dernières années auraient produit actuellement un corps de connaissances fiables sur lequel il serait alors possible de s'appuyer pour enseigner. Cependant, la recherche sur le sujet est aujourd'hui tellement abondante qu'un travail de synthèse des 
résultats est devenu nécessaire pour en dégager les éléments de convergence; un travail critique et de mise en perspective s'est avéré également tout aussi essentiel afin de mieux en saisir la portée et en guider l'utilisation. (p. 8)

\subsection{Constat en rapport aux méthodes de synthèse}

Comme nous venons de le voir de façon succincte, il existe plusieurs méthodes disponibles au chercheur qui désirerait tirer profit d'un ensemble d'études déjà complétées. Toutes ces techniques ont des prémisses, des avantages, des limites, des finalités similaires ou contrastées. Pour la plupart, elles ont aussi comme caractéristiques de ne pas être suffisamment définies dans leurs différents aspects qui en font des méthodes de recherche scientifique.

Concernant ce dernier point, dans notre recherche, nous tentons l'exercice de clarifier la métasynthèse, notamment en la comparant avec la méta-analyse qualitative. À la section suivante, nous attardons à la réflexion et à la sélection de critères descriptifs permettant de bien définir la métasynthèse dans son ensemble.

\section{LES DIMENSIONS DESCRIPTIVES PROPRES À UNE MÉTHODE DE RECHERCHE}

L'objectif de la présente recherche consiste à expliciter la métasynthèse et à la comparer avec la méta-analyse qualitative. Concrètement, nous voulons offrir à la communauté scientifique un écrit qui fait le point sur la métasynthèse et sur lequel se baser pour en comprendre les grandes lignes. Pour ce faire, il convient de s'appuyer sur des critères descriptifs pertinents et révélateurs. L'exercice de dégager ces caractéristiques et d'expliciter leur choix est au coeur des prochains paragraphes. Pour ce faire cet exercice, nous nous basons essentiellement sur deux textes. De fait, peu d'ouvrages traitent des méthodes de recherche sous l'angle descriptif. Ainsi, c'est pour orienter cette démarche descriptive que nous tentons de trouver des critères descriptifs dans les textes recensés, question de nous en inspirer. 
En premier lieu, il y a le texte utilisé dans la cadre de la section précédente, Synthesing qualitative and quantitative evidence: a review of possible methods (DixonWoods et al., 2005). Dans cet article, les auteurs ont inclus à la fin de celui-ci un tableau synthèse reprenant brièvement chacune des méthodes de recherche abordées, et ce, sous quatre thèmes. Le premier thème, Outline of approach, se veut un résumé très court de la technique abordée. Le deuxième, Sub-forms and developments, présente l'orientation que peut prendre la méthode en question (theory-led, data-driven, theory driven, etc.), ou encore les variantes possibles. Le troisième, Some problems, expose les limites alors que le quatrième et dernier thème, Some strengths, liste les principales forces de la méthode décrite. En second lieu, nous avons également transcrit un tableau de Van der Maren (1996) à la page 18, lequel propose une définition d'une méthode de recherche. Les éléments énumérés sont pertinents et peuvent servir aux fins de notre travail. Les caractéristiques les plus fondamentales sont sans aucun doute l'intention, le but et l'objectif de la recherche. Van der Maren évoque également des techniques au cœur de la méthode, une autre composante très importante.

Pour l'étude que nous avons effectuée, nous nous sommes donc inspiré de ces auteurs pour être en mesure de proposer des critères pertinents à une description relativement pointue d'une méthode de recherche. Ainsi, nous avons repris des thèmes utilisés par Dixon-Woods et al. (2005) et Van der Maren (1996) dans nos critères descriptifs, parfois tels quels, ou encore décortiqués à travers des concepts que nous croyions plus englobants et plus poussés. Dans l'optique de ce que les différents auteurs ont proposé comme thèmes pour caractériser des méthodes de recherches, et selon les finalités de cette étude, le tableau 4 présente les thèmes retenus comme critères descriptifs: 
Tableau 4

Thèmes descriptifs retenus aux fins de l'analyse d'une méthode de recherche

Définition: selon les divers textes analysés et leurs auteurs, définition de la méthode analysée;

Buts: les buts, les finalités visées par cette méthode, par son utilisation par les chercheurs;

Origine: d'où vient cette méthode, les raisons de sa création, les chercheurs qui ont contribué à son éclosion, dans quel courant, etc.;

Pertinence: la pertinence, la justification d'utiliser cette méthode, les critères pouvant mener à son utilisation, etc.;

Composantes: les composantes, les procédures, les différentes parties de méthode étudiée;

Étapes: les étapes pour mener à terme une étude effectuée avec la méthode analysée;

Procédures: à l'intérieur des étapes, les techniques à employer;

Limites: les éléments à prendre en considération lorsqu'on envisage de faire une recherche à l'aide de cette méthode;

Forces: les éléments qui donnent du poids à l'utilisation de cette méthode;

Exemples: des exemples concrets résultants de l'utilisation de cette méthode.

Le chapitre suivant, la méthodologie, présente la procédure que nous avons suivie pour analyser les textes relevés lors de notre recension des écrits de même que la forme qu'a prise la présentation et la discussion des résultats. 


\section{CHAPITRE TROIS \\ LA MÉTHODOLOGIE}

Le troisième chapitre de ce mémoire est consacré à expliquer comment nous avons analysé les textes recueillis lors des recensions dans l'optique de répondre à l'objectif de recherche proposé qui est de comparer la méta-analyse qualitative et la métasynthèse pour ensuite rendre explicite à la communauté scientifique en éducation la méthode de recherche de la métasynthèse de manière à faciliter son utilisation. Dans le chapitre précédent, nous avons vu qu'il existait quelques méthodes permettant de revenir sur des études, dont l'analyse de contenu. Nous allons utiliser cette méthode pour nos besoins, mais il est bon de rappeler que les textes que nous avons analysés ne sont pas des recherches sur la métasynthèse et la méta-analyse qualitative, mais bien des textes traitant de ces méthodes, ou encore des recherches dont la méthodologie est l'une de ces approches. L'exercice est donc quelque peu différent de ce qui est présenté brièvement dans le tableau 3. Pour démontrer comment nous avons mis en oeuvre l'analyse de contenu dans notre recherche, nous l'abordons dans la première section du présent chapitre. Dans la deuxième section, nous explicitons ce que nous cherchions dans les textes étudiés, soit les thèmes retenus pour l'analyse de contenu. Finalement, la troisième section explique brièvement la forme qu'a prise la présentation des résultats de notre recherche au quatrième chapitre.

\section{L'ANAL YSE DE CONTENU}

Les données recueillies dans le cadre de cette recherche sont des éléments textuels extraits des articles et du livre découlant des recensions des écrits effectuées. Dans les prochains paragraphes, nous allons décrire *l'analyse de contenu, méthode que nous avons employée dans le cadre de cette recherche, et la manière dont nous l'avons utilisée dans le cadre spécifique de notre mémoire. 
Pour expliciter la méthode de recherche que nous avons utilisée, nous nous sommes essentiellement basé sur un écrit de L'Écuyer (1988) intitulé L'analyse de contenu : notion et étapes. Nous avons choisi ce texte car l'auteur présente l'analyse de contenu d'une manière qui sied bien aux objectifs visés ici. En effet, notre intention n'est pas tant d'interpréter les textes dans le but d'en faire ressortir une compréhension nouvelle, mais davantage d'avoir recours à une méthode plus rigoureuse que la simple lecture accompagnée d'annotations pour faire ressortir les éléments importants des textes. Ainsi, à la différence de plusieurs autres auteurs sur le sujet (par exemple, Muchielli (2003), Paillé (2003) et Sabourin (2003) qui abordent davantage l'analyse de contenu par ses finalités interprétatives, L'Écuyer présente cette méthode sous un angle méthodique et structuré. Puisque nos besoins d'analyse ne requièrent pas une interprétation en profondeur des propos contenus dans nos textes et que nous voulons davantage faire une analyse documentaire à l'aide de concepts clés, l'approche de L'Écuyer convient à notre intention de recherche.

Pour conceptualiser ce qu'est l'analyse de contenu, L'Écuyer emprunte à Muchielli (1979) une première définition, très brève: « méthode visant à découvrir la signification du message étudié » (p. 50). Jusqu'ici, sans entrer trop profondément dans l'aspect interprétatif de l'analyse de contenu, cette définition satisfait à nos exigences. L'Écuyer précise que: «[...] c'est une méthode de classification ou de codification dans diverses catégories des éléments du document analysé pour en faire ressortir les différentes caractéristiques en vue d'en mieux comprendre le sens exact et précis. " (p. 50) La classification dont il est question ici sera traitée à la prochaine section.

Même si nous nous basons sur L'Écuyer pour traiter de l'analyse de contenu, il est intéressant de voir ce qu'en disent d'autres auteurs. Cet exercice nous permet surtout de faire un constat, à savoir qu'il n'existe pas de définition et de mode d'utilisation uniques de l'analyse de contenu. Esterberg (2002) s'attarde dans un premier temps au concept de comptage. Comme il donne en exemple, une analyse de contenu peut simplement être de dénombrer la quantité de photos d'hommes et de femmes dans un type de publication. Le 
comptage peut également se faire sur certains mots, termes, thèmes, concepts. Les apparitions répétées de ceux-ci témoigneront généralement d'un niveau d'importance. S'éloignant du principe du dénombrement, l'analyse de contenu peut aussi avoir comme mandat de rechercher le sens dans les textes étudiés, par exemple, en examinant des bandes dessinées propres à un thème (selon un exemple de Esterberg, la fête des Pères) sur une longue période (1940 à 1999) pour dépeindre l'évolution d'un phénomène (la conception du fait d'être père, le fatherhood). L'analyse de contenu vue sous ces angles est également partagée par Bernard (2000) et Patton (2002), ce dernier admettant volontiers que cette méthode de recherche n'est pas un concept clair. Il propose malgré tout la définition suivante: « De façon générale, [...], l'analyse de contenu réfère à tout effort de réduction et de création de sens de données qualitatives, prenant un grand corpus qualitatif pour tenter d'en identifier une logique et un sens profond. » (p. 453, traduction libre) ${ }^{24}$ Cette définition est pertinente au processus que nous désirons entreprendre, soit de faire ressortir certains thèmes des textes analysés, sans pour autant porter une attention exagérée à la quantification de ceux-ci.

\section{LES THÈMES D'ANALYSE}

En reprenant le tableau 4 du précédent chapitre, nous avons convenu d'une série de thèmes qui nous intéresse à l'égard de la métasynthèse (de façon plus approfondie), mais que nous avons également repris pour la méta-analyse qualitative (de façon moins poussée). L'instrument de cueillette des données utilisée pour atteindre notre objectif est la grille d'analyse $\mathrm{e}^{25}$. Nous avons tout d'abord recherché ces thèmes dans les cinq textes qui traitent de la méta-analyse qualitative, pour ensuite effectuer une seconde analyse de contenu sur les 12 autres publications recensées liées à la métasynthèse. Dans les deux cas, des extraits qui répondent aux exigences de la grille ont été répertoriés, utilisés et analysés

\footnotetext{
24 "More generally, however, content analysis is used to refer to any qualitative data reduction and sensmaking effort that takes a volume of qualitative material and attempts to identify core consistencies and meanings."

${ }^{25}$ La grille d'analyse est présentée dans l'Annexe E
} 
pour obtenir les résultats. De façon plus théorique, les extraits sélectionnés, ou plutôt les textes desquels ils sont tirés, sont identifiés par L'Écuyer comme étant du *contenu manifeste. Il s'agit « [...] du matériel brut faisant l'objet de l'analyse, laquelle porte alors directement et exclusivement sur ce qui a été ouvertement dit ou écrit, tel quel, par le répondant " (p. 51). Dans notre cas, il ne s'agit pas de répondants à une entrevue, mais plutôt d'auteurs qui ont écrit:

- des articles dont la méthode de recherche mise en oeuvre est la méta-analyse qualitative;

- des articles dont la méthode de recherche mise en oeuvre est la métasynthèse;

- des textes portant sur la métasynthèse.

Habituellement, dans le but d'optimiser une analyse de contenu et l'utilisation d'une grille d'analyse, une série d'étapes encadrent le processus l'analyse, comme le présente L'Écuyer:

\section{Tableau 5}

Étapes d'une analyse de contenu (L'Écuyer, 1988)

1. Lectures préliminaires et établissement d'une liste d'énoncés.

2. Choix et définitions des unités de classification: types d'unités, définitions et critères de choix.

3. Processus de catégorisation et de classification: définition d'une catégorie, sous-étape de classification, qualités essentielles des catégories.

4. Quantification et traitement statistique.

5. Description scientifique: analyse quantitative et analyse qualitative.

6. Interprétation des résultats.

Source: L'Écuyer, L'analyse de contenu : notion et étapes, p. 54-55

Or, comme nous l'avons mentionné auparavant, les analyses de contenu que nous avons réalisées sont des analyses de contenu manifeste, soit des extraits de textes. Par conséquent, certaines étapes que propose L'Écuyer n'ont pas été mises en oeuvre. L'étape 1 ne cause pas problème, bien que les énoncés soient tributaires des choix préétablis dans l'étape 2. En effet, nous savions déjà au début du processus de l'analyse de contenu ce que nous désirions dégager de nos textes. Par conséquent, les étapes 2 et 3 ont été simplifiées. 
L'étape 4 n'a pas été mise en application telle que proposée, notre intention n'étant pas d'évaluer statistiquement la présence de propos identiques ou similaires dans leur sens. Nous voulons plutôt présenter un portrait de la métasynthèse à travers les thèmes de notre grille d'analyse, ce qui évacue du même coup l'aspect quantitatif de l'étape 5 . L'interprétation des résultats, introduite à l'étape 6, s'est traduite par un texte tel que décrit dans la section suivante.

\section{LES DONNÉES RECUEILLIES AVEC LA GRILLE}

Dans cette section, des extraits de textes sur la métasynthèse, la méta-analyse qualitative et la méta-étude sont répertoriés dans des sous-sections identifiées selon les thèmes déterminés par la grille d'analyse de contenu. Le contenu présenté ici est un contenu manifeste, c'est-à-dire émanant directement des textes. Puisque des constats surgissent toujours lors d'un processus de cueillette de données et de l'analyse de celles-ci, quelques modifications concernant les rubriques originales de la grille ont été apportées. Elles seront expliquées au fur et à mesure de la présentation du contenu dégagé.

\section{Définitions}

Dans cette catégorie, nous avons regroupé les passages qui expliquent d'une manière ou d'une autre la métasynthèse, la méta-analyse qualitative, la métaétude de même que diverses composantes de ces méthodes. Il est à noter que dans les passages définissant ces concepts, il est parfois question du but de la méthode, de son utilité. Cette catégorie est par ailleurs la plus importante et c'est ce que nous recherchions, dans le but de pouvoir dresser un portrait le plus global possible de la métasynthèse selon la vision de plusieurs auteurs. 


\section{$1.1 \quad$ Extraits de textes}

«Meta-analysis is the critical review and statistical combination and evaluation of the results of previous research [...].» (Yu et al., 2005, p. 268)

«Umbrella term referring to the synthesis of findings across multiple qualitative reports to create a new interpretation.» (Finfgeld, 2003, p. 895)

«Metasynthesis is neither a systematic review of the literature (Schreiber et al., 1997; Sherwood, 1999) nor the collating (i.e., codifying) of research findings (Sherwood, 1997). In addition, it is not the aggregation (i.e., summing) of research outcomes or a concept analysis. Rather, "it is the bringing together and breaking down of findings, examining them, discovering the essential features, and, in some way, combining phenomena into a transformed whole (Schreiber et al., 1997, p. 314). A metasynthesis is a complete study that involves rigorously examining and interpretting the findings (versus the raw data) of a number of qualitative research studies (Jensen et Allen, 1996) using qualitative methods » (Finfgeld, 2003, p. 894)

«Metasynthesis is a method inherently designed to facilitate knowledge development. As such, it can be understodd as a form of discourse that contributes to a fuller understanding of the phenomenon of interest. Particularities are retained, and the reconstruction enhences the complexity. It might tehrefore be considered a deconstruction for the purpose of restructuring an order from which to operate. In this way, metasynthesis functions as a translation, a means to grasp the particulars within the wholes. Applied to a body of research, it reduces, but does not eliminate, uncertainty.» (Jensen, 2004, p. 1345)

«This meta-synthesis differs from the simple accumulative logic or averaging accross studies. The uniqueness and holism of accounts is retained even though synthesized in the translation; texts are compared to create a holistic interpretation.» (Jensen et Allen, 1996, p. 554) 
«There is no standard method for combining qualitative studies. The term qualitative meta-analysis covers a range of methods, from reanalysis of primary data collected in multiple studies to analysis of results reported in published articles. In this article, we will use the latter approach, drawing on the Schutz framework of constructs. Our method is based on the metaethnography proposed by Britten and Campbell and colleagues, and first described by Noblit and Hare. We prefer the term meta-analysis, because the studies we are analyzing are not ethnographies. We also compared our findings with previously published national guidelines.» (Feder et al., 2006, p. 23)

«Three types of metasynthesis have been proposed: theory building, theory explication, and descriptive study.» (Finfgeld, 2003, p. 894)

«Metasynthesis: Umbrella term referring to the synthesis of findings across multiple qualitative reports to create a new interpretation. Proposed types of metasyntheses include theorybuilding, metastudy, ground formal theory, theory explication, and descriptive study» (Finfgeld, 2003, p. 895)

«Metastudy is another type of theorybuilding metasynthesis. This variation is composed of three distinct types of analysis, which are then brought together to create a new theoretical interpretation.» (Finfgeld, 2003, p. 895)

«Descriptive metasynthesis involves the synthesis of qualitative findings and results in a comprehensive analysis of phenomena (Schreiber at al, 1997). In contrast to the theoretical explication approach, which focuses on the analysis of a single concept, descriptive metasynthesis looks more broadly at phenomena [...]» (Finfgeld, 2003, p. 897)

«In recent years, the term metasynthesis has evolved from its original position as a distinctive approach in comparison to others to attain some popularity as the most comprehensive generic term representative of this collection of approaches, and so it is in 
that particular context that we refer to it here. As our argument unfolds, however, the reader will notice the terminological land mines that complicate the study of these kinds of approaches, and we will therefore attempt to distill from the terminological options that which is central to the problem of meta-approaches. We see all metasynthesis, regardless of its terminological labels, as representing a family of methodological approaches to developing new knowledge based on rigorous analysis of existing qualitative research findings. And as with all families, disputations and dynamics are to be expected.» (Thorne et al., 2004, p. 1343)

«In qualitative health research, we have come to understand that metasynthesis must be quite different from simple accumulative logic or averaging across studies. The goal is clearly interpretive, not mere aggregation to achieve unity; it is not a summary portraying the lowest common denominator. Metasynthesis is not a method designed to produce oversimplification; rather, it is one in which differences are retained and complexity enlightened. The goal is to achieve more, not less. The outcome will be something like a common understanding of the nature of a phenomenon, not a consensual worldview. The result of a qualitative metasynthesis is not "the truth" or "the answer"; neither is it simply a narrative or a systematic review. Metasynthesis is quite different from secondary analysis, whereby data are reanalyzed with a new technique or new question. It cannot negate or violate the context or paradigm of the primary studies and, therefore, does not compensate for limited scientific rigor within a body of research. [...] Metasynthesis is a method inherently designed to facilitate knowledge development. As such, it can be understood as a form of discourse that contributes to a fuller understanding of the phenomenon of interest. Particularities are retained, and the reconstruction enhances the complexity. It might therefore be considered a deconstruction for the purpose of restructuring an order from which to operate. In this way, metasynthesis functions as a translation, a means to grasp the particulars within the wholes. Applied to a body of research, it reduces, but does not eliminate, uncertainty.» (Jensen in Thorne et al., 2004, p. 1346) 
"What we call now metastudy is a particular form of metasynthesis is a particular form of metasynthesis grounded within a tripartite analytic process that includes metatheory, metamethod, and meta-data-analysis» (Thorne in Thorne et al., 2004, p. 1355)

«Looking back over what I have thought and written, I have come to see qualitative metasynthesis as signifying only one kind of systematic review and type of qualitative research integration. Indeed, in everything I write from now on, I plan to use the phrase qualitative research integration to refer to empirical studies directed toward the combination of research findings in reports of qualitative studies.» (Sandelowski in Thorne et al., 2004, p. 1357)

«Accordingly, a qualitative metasummary is a quantitatively oriented aggregation of qualitative findings that are, in turn, topical or thematic summaries or surveys of data. Metasummaries are integrations that are approximately equal to the sum of parts, or the sum of findings across reports in a target domain of research. They do not stray far from the findings as given in research reports and reflect a quantitative logic: to discern the frequency of each finding and to find in higher frequency findings the evidence of replication believed to be foundational to validity. Metasummary techniques involve the extraction and reduction of data and the calculation of effect sizes.

In contrast, qualitative metasynthesis is an interpretive integration of qualitative findings that are themselves interpretive syntheses of data, including phenomenologies, ethnographies, grounded theories, and other integrated and coherent descriptions or explanations of phenomena, events, or cases (Sandelowski \& Barroso, 2003a). Such interpretive syntheses of data are considered to be hallmarks of qualitative research. Metasyntheses are integrations that are more than the sum of parts, in that they offer novel interpretations of findings. These interpretations will not be found in any one research report but, rather, are inferences derived from taking all of the reports in a sample as a whole. Metasyntheses offer a coherent description or explanation of a target event or experience. Such interpretive integrations require researchers to piece the individual syntheses constituting the findings in individual research reports together to craft one or 
more metasyntheses. Their validity resides not in a replication logic but, rather, in an inclusive logic whereby all findings are accommodated and in the craftsmanship exhibited in the final product. Metasynthesis methods include, for example, constant comparison, taxonomic analysis, the reciprocal translation of in vivo concepts, and the use of imported concepts to frame data (Sandelowski \& Barroso, 2003d). Qualitative metasummaries can serve as an empirical foundation for qualitative metasyntheses by which reviewers can prepare findings for metasynthesis and maximize the descriptive and interpretive validity of the integrations they produce. Summing up these summaries allows reviewers better to see ways to link them.» (Sandelowski in Thorne et al., 2004, p. 1358)

«For me, metastudy is a species of scholarship different from research integration, which I see as having more limited empirical goals but greater immediate utility for practice than metastudy.» (Sandelowski in Thorne et al., 2004, p. 1359)

«Rather than simply appropriating the data of multiple researchers to make "larger arguments" about phenomena, we envision metasynthesis evolving into a complex set of strategies with which scholars in the health research field render the warrants for their qualitatively derived assertions in a manner that is increasingly accessible, auditable, and transparent. [...] The products that derive from such intellectual approaches will be integrative, coherent, and illuminating but rarely "factual." We envision, therefore, that the truths deriving from qualitative metasynthesis will be inherently more convincing when they are articulated in a manner that acknowledges their boundedness within temporal, spatial, and epistemological locations.» (Thorne et al., 2004, p. 1361)

"Although all qualitative research, narrative integrations of quantitative research, and broad overviews of knowledge fields entail synthesis, or some combination of two or more entities, qualitative metasynthesis is a distinctive category of synthesis in which the findings from completed qualitative studies in a target area are formally combined. Qualitative metasynthesis constitutes a specific kind of data-driven research that is analogous to quantitative meta-analysis in its intent systematically, as opposed to 
impressionistically (Fawcett, 1999), to combine the findings in a target domain of scientific research.» (Sandelowski et Barroso, 2003, p. 782)

"Qualitative metasynthesis - as it is conceived in the few articles on this subject [...]- is a form of metastudy, that is, study of the processes and results of previous studies in a target domain that moves beyond those studies to situate historically, define for the present, and chart future directions in that domain. In metastudies, the researcher seeks not only to combine the results of previous studies but also to reflect on them (Thao, 1991, pp. 377-378). Like phenomenology, ethnography, and grounded theory, the terni, qualitative metasynthesis refers both to an interpretive product and to the analytic processes by which the findings of studies are aggregated, integrated, summarized, or otherwise put together (Estabrooks, Field, \& Morse, 1994; Jensen \& Allen, 1996; Kearney, 1998a; Noblit \& Hare, 1988; Sandelowski, Docherty, \& Emden, 1997; Schrieber, Crooks, \& Stern, 1997). Although it can be considered an analogue to meta-analysis (Glass, McGaw, \& Smith, 1981 ) in that there is "a shared interest in synthesizing empirical studies" (Noblit \& Hare, 1988 , p. 10) and a shared desire to use a systematic, comprehensive, and communicable approach to research integration, qualitative metasynthesis is not about averaging or reducing findings to a "common metric" (Wolf, 1986, p. 33). [...] Instead, the aim of qualitative metasynthesis is to create larger interpretive renderings of all of the studies examined in a target domain that remain faithful to the interpretive rendering in each particular study. A prime directive for qualitative researchers, no matter what their method or research purpose, is to preserve the integrity of each sampling unit or case (Sandelowski, 1996). In qualitative metasynthesis projects, this prime directive entails preserving the integrity of and the richness of findings in each individual study.» (Sandelowski et Barroso, 2003, p. 784-785)

«Two kinds of interpretative syntheses of findings from qualitative studies have been attempted. One involves the integration of findings from multiple analytic paths pursued within one program of research by the same investigator(s). [...] A second kind of effort, and the one we will focus on in this project, involves the interpretive synthesis of 
qualitative findings across studies conducted by different investigators.» (Sandelowski et Barroso, 2003, p. 786)

"This meta-synthesis differs from simple accumulative logic or averaging across studies. The uniqueness and holism of accounts is retained even though synthesized in the translation; texts are compared to create a holistic interpretation. Therefore, the goal of meta-synthesis is interpretative, not aggregative. Rather than the outcome being one of establishing predictive theory, the goal is understanding or interpretive explanations of the phenomenon. Meta-synthesis would thus continue to be firmly based in the interpretive paradigm. Yet, are human experiences comparable and thus suitable for synthesizing?

In the interpretive paradigm, "reality is deemed multiple and constructed, rather than singular and tangible" (Sandelowski, 1993, p. 3). As a result, given the same qualitative task, no two researchers will produce the same result; there are inevitable differences in perspectives and styles. Consequently, this poses another question when synthesizing qualitative findings. Although there can be multiple noncontradictory descriptive and explanatory daims about any phenomenon, those interpretations must correspond in relevant aspects to the phenomenon described. Furthermore, words have more than one meaning when constructed outside their use in a determinate context (Ricoeur, 1983). Concepts and ideas are invented (rather than discovered), yet these inventions are rooted in the real world. Meaning is not inherent in raw data, but rather explanatory arguments are constructed from data (Dzurec \& Abraham, 1993). What is being sought is a consensus on the nature of the phenomenon. A synthesis of qualitative accounts should grab the essence of the phenomenon, presenting away to achieve a "fuller knowing" to advance knowledge (Sandelowski, 1993)» (Jensen et Allen, 1996, p. 554)

«Throughout this process, rigor is essential to achieve credible and consistent descriptions of the phenomenon. The general themes of credibility, auditability, and fittingness persist as criteria for scientific rigor (Cuba \& Lincoln, 1981; Sandelowski, 1986). The truth value of qualitative account synthesis would reside in the discovery of human phenomena or experiences as they are lived and perceived by subjects, rather than 
in the verification of a priori conceptions of those experiences. Thus a meta-synthesis is rooted in the original data and is credible when it re-presents such faithful descriptions or interpretations of a human experience that the people having that experience would immediately recognize it from those descriptions or interpretations as their own. Consequently, achieving credible interpretations is fostered if original studies provide exemplars.» (Jensen et Allen, 1996, p. 556)

"The prefix "meta" has now appeared in many disciplines in the social sciences. In general, "meta" is used in the sense of "after," "about," and "beyond. Meta-study refers, first of all, to studies that come after some other studies, that is, some nonmeta-studies must exist before meta-study becomes possible. Meta- study involves studies about (or of) other studies. After a given set of studies is done, at least two different types of studies can follow: (1) restudying the same phenomenon that was previously studied (replication, for example) and (2) studying the results and the processes of the previous studies. Meta-study belongs to the latter category. It is occasionally referred to as a second-order study: while a first-order study analyzes a given phenomenon, say, X, a second-order study analyzes the study of X (McMullin 1970). Meta-study transcends or goes beyond previous studies. Meta-study differs from other forms of second-order studies (e.g., historical, logical, and esthetic analyses of first-order studies) in that it seeks not only to synthesize the results of previous studies but also to reflect upon the processes involved in previous studies in terms of "where we are and where we are going" (Fuhrman and Snizek 1990:27).

Metatheory in sociology is the study of extant sociological theory. The difference between sociological theory and metasociological theory is the following: while the former theorizes about the social world, the latter theorizes about theories of the social world. Metatheory thus has indirect relevance to the study of the social world. Ritzer (1990a) classifies metasociological theorizing into three branches according to differences in their end products. The first branch is metatheorizing as a means of attaining a deeper understanding of theory $(\mathrm{Mu})$ and it involves the effort to uncover the underlying structures of extant sociological theory. The second branch is metatheorizing as a prelude to theory development $(\mathrm{Mp})$ which involves the study of sociological theory in order to 
produce new sociological theory. Finally, there is metatheorizing as a source of overarching perspectives (Mo) in which the study of theory is oriented to the goal of producing a perspective that overarches some part or all of sociological theory. All three branches take extant sociological theory as their subject matter and examine it reflexively.

Metamethod in sociology is the study of extant sociological research methods. According to Furfey ([1953] 1965), metamethod consists of three types of studies: (1) the examination of the methodological presuppositions necessary for carrying out sociological research; (2) the evaluation of extant sociological research methods in terms of their weaknesses and strengths; and (3) the codification of new procedural rules for sociological research. 4 Metamethod, in this sense, carries essentially the same connotation as that of methodology which "uses reason as a judge of all methods, by weighing the rigor, epistemological soundness, and fruitfulness of different approaches. Particularly important is to judge how ap- propriate a method is to a particular subject" (Richman 1983:136).

Meta-data-analysis in sociology is the study of the results of sociological data analysis. The results of data analysis are findings obtained through the analysis of raw materials, i.e., data that are collected in the field. While data analysis processes "raw data," meta-data-analysis processes the "processed data." Meta-data-analysis is not the same as data-reanalysis that seeks to analyze the same set of raw data over again using different procedures or for different purposes. Instead, meta-data-analysis is the analysis of the results of previous analysis or "the analysis of analyses" (Glass 1976). Meta-data-analysis consists of: (1) the study of the underlying assumptions of various data-analytic procedures; (2) the comparison of different forms of data in terms of their quality and utility; and (3) the synthesis of the findings of a range of research studies that are related to the same phenomenon.» (Zhao, 1991, p. 377-379)

«Meta-theory creates the context in which the implications of a range of theoretical approaches that have influenced the body of knowledge can be considered and evaluated. In addition, new theoretical alternatives that might account for a more comprehensive, accurate, or credible interpretation of the phenomenon can be synthesized. As has been discussed in earlier chapters, each primary study can be best understood when it is 
considered in its demographic, theoretical, and historic context. This step allows the researcher to understand in a different way why primary researchers may have obtained different findings at different times.»(Paterson et al., 2001, p. 117)

"When insights from meta-data-analysis, meta-method, and meta-theory are brought together, the meta-study researcher will typically en- counter many more questions than answers. Although a synthesis of knowledge seems quite plausible on the basis of studies using similar samples, similar methods, and similar theoretical understandings, we have argued quite strongly in this discussion that this type of synthesis generally represents mere aggregation. In aggregating findings of like in- quiries, we are not really provided any more confidence in our conclu- sions or better understandings of a phenomenon; instead, we learn that if we ask questions in a particular way, we will get a predictable set of answers. In contrast, meta-synthesis capitalizes on diversity of context, method, and theoretical orientation to allow the possibility of a richer, deeper, and more multifaceted way of theorizing about a phenomenon.» (Paterson et al., 2001, p. 119)

\section{Buts}

Dans cette catégorie de la grille d'analyse, nous cherchions à déceler des éléments expliquant un peu plus précisément l'utilité d'effectuer une métasynthèse, une métaanalyse qualitative, une méta-étude ou l'une des composantes de ces méthodes. Comm nous l'avons indiqué dans l'introduction de la précédente catégorie, des éléments «de buts» sont souvent inclus dans des passages traitant des définitions des méthodes ci-haut mentionnées, les deux catégories étant ainsi complémentaires. 


\subsection{Extraits de textes}

«Because the synthesis of qualitative findings is interpretative rather than aggregative [...]» (Finfgeld, 2003, p. 894)

«The appeal of meta-synthesis lies in our hunger for more true, more accurate, or more real explanations of phenomena and more coherent ways to make sense of them. To this point, we have been locating meta-study within the context of constructivist enterprises. In keeping with this context, we fully acknowledge that meta-synthesis is similarly a social construction. That said, however, we also believe that the purpose of engagement in meta-synthesis is not merely to add new ideas to the collection of available constructions. Rather, we are convinced that underlying the notion of social construction is a competing ideal of social responsibility, morality, and accountability. [...] The object of a meta-synthesis, then, is not simply to report similarities within the research literature in relation to a particular phenomenon, or to account for common patterns within the available knowledge, or to reduce the available understandings to the lowest common denominator. Rather, it is to dig below the surface of what is currently understood, to draw on the most thorough analysis possible to deconstruct the validity of the ideas that are currently in favor, and to emerge with the kernel of a new truth, a better kind of understanding, or a more socially responsible form of theorizing something. In so doing, it creates the possibilities of looking beyond, imagining something better, and contributing to a more complex and infinitely interesting scholarship. » (Paterson et al., 2001, p. 110-111)

«The synthesis of findings across studies is a type of secondary analysis particular to qualitative research, which provides a powerful approach to theory development (Estabrooks, Field, \& Morse, 1994)» (Jensen et Allen, 1996, p. 553)

«[...] summarize and synthesize the existing distance learning studies in order to understand what works and what does not in higher education distance learning programs.» (Chen et Turner, 2001. p. 2) 
"As it is variously conceived in the literature and operationalized in the practice of research, qualitative metasynthesis does not necessarily entail any actual synthesis, or integration, at all (e.g., of findings, methods, or frames of reference) but, rather, an inventory, description, comparison, or critique of them.» (Sandelowski in Thorne et al., 2004, p. 1358)

"Given an ecological framework for qualitative meta-synthesis, then the concepts of "meta-synthesis" and "triangulation" become foundational. The work of (Zhao, 1991) and the application of his work by (Patterson, Thorne, Canam, \& Jillings, 2001) suggest that a meta-synthesis should include an analysis of theory (meta-theory), an analysis of method (meta-method) and an analysis of findings (meta-analysis). An aggregation of findings alone does not lead to sufficient understanding of the research phenomenon. The analysis of the role of theory and methods within the studies under examination must also be a part of the synthesis procedure.» (Banning, s.d., p. 1)

«The goal of metasynthesis is to produce a new and integrative interpretation of findings that is more substantive than those resulting from individual investigations. This methodology allows for the clarification of concepts and patterns, and results in refinement of existing states of knowledge and emergent operational models and theories (Sherwood, 1999).» (Finfgeld, 2003, p. 894)

"Beyond meta-data-analysis, meta-synthesis can take the researcher into the domain of the assumptions underlying a body of research findings and the interpretations that have been made about it. In particular, it can extend the interpretation from what has been studied to what has not and can permit speculation about why this might be so. Although meta-data-analysis is a useful exercise in its own right, we believe that the process of trying to work with both aggregations and contradictions arising from qualitative data is integral to a truly exciting and illuminating meta-synthesis. When new interpretations are presented in the context of the old and elucidate relationships between 
the various discrete components of the old, they can be said to truly extend knowledge in the field.» (Paterson et al., 2001, p. 114-115)

«In most meta-study projects, it will not be possible to predict the degree to which new theory can be synthesized until the products of meta-data-analysis, meta-method, and meta-theory are individually and collectively interpreted. Typically, as we have observed in all our meta-study projects, the process inspires more pessimism than optimism because of the gaps, flaws, and limitations it can reveal within a body of qualitative inquiry. It must be recognized that this rigorous analytic process involves an extended critical deconstruction of what is known and how it is known, and many analysts will find it difficult to get past the conclusion that "we know nothing!" In our consultations with others who have ventured into this territory, we understand that despair about truth and knowledge is a frequent experience and can become an insecure foundation on which to try to generate new theorizing.

We do believe, however, that the key to a successful meta-synthesis effort lies in recognizing that small gains can be as important as larger ones and that better ways of theorizing about narrow aspects of a field may be more useful in the long run than are completely original grand theories. In our chronic illness meta-study project, for example, we came to realize that our dream of creating a comprehensive new way of theorizing chronic illness was remarkably naive. At the same time, however, we discovered several current claims in relation to chronic illness whose contradictions could be reconciled and whose theoretical applications could be extended. [...] Although these may seem like minor theoretical accomplishments in the larger scheme of things, we are satisfied that they represent something larger than our individual pieces of research could possibly attain. We believe that meta-study has given us a much more comprehensive and complete way of understanding the field, of putting research products in context, and of imagining the truly magnificent kinds of theorizing that may become possible in the future.» (Paterson et al., 2001 , p. 120-121) 


\section{Origine}

Nous voulions, à l'aide de cette catégorie de la grille d'analyse, déterminer un moment, voire un contexte, expliquant l'émergence de la métasynthèse puisqu'il s'agit d'un type de méthode de recherche assez particulier. Nous avons obtenu quelques pistes de réponse, mais il ne s'agit pas d'un aspect régulièrement abordé lorsqu'on parle d'une méthode de recherche.

\subsection{Extraits de textes}

«[...] post-positivists scholars expressed considerable interest in synthesizing diverse theorical and disciplinary positions into grand theories.» (Paterson et al., 2001, p. 1)

«Although metasyntheses and variations thereof were undoubtedly conducted prior to 1994 , this year marks a time in which these types of investigations were encouraged and researchers began to make more concerted efforts to explicate their methodologies» (Finfgeld, 2003, p. 894)

«Louise Jensen begins by locating the metasynthesis project within the context of quantitative meta-analysis, which represents her own origins within this field» (Thorne et al., 2004, p. 1345)

\section{Pertinence}

Par cette catégorie de la grille d'analyse, nous désirions dégager le point de vue d'auteurs, de chercheurs qui s'exprimeraient en se portant à la défense de la métasynthèse, 
ou en en faisant la promotion. Nous avons trouvé peu de passages très explicites en ce sens, mais les catégories «Définitions», «Buts» et «Forces» peuvent très bien se combiner à celle-ci pour mettre en relief la pertinence de la métasynthèse.

\subsection{Extrait du texte}

«[...] it is to dig below the surface of what is currently understood, to draw on the most thorough analysis possible to deconstruct the validity of the ideas that are currently in favor, and to emerge with the kernel of a new truth, a better kind of understanding, or a more socially responsible form of theorizing something.» (Paterson et al., 2001, p. 111)

\section{Composantes}

Encore ici, ne sachant pas trop ce que nous allions découvrir sur la métasynthèse, nous voulions proposer une catégorie dans la grille d'analyse qui ferait le point sur les éléments qui composent la métasynthèse. Nous avons trouvé quelques extraits en ce sens, traitant surtout de la métathéorie, de la métaméthode et de la méta-analyse de données, tous dans le cadre d'une métasynthèse sous forme de méta-étude. À noter que d'autres éléments se référant à ces composantes sont présentés dans les catégories «Définitions» et «Buts» lorsqu'il est question de la méta-étude.

\subsection{Extraits de textes}

(Métaméthode) «Although analytic methods in qualitative research have certain common practices (e.g., establishing codes to represent data), each analytic approach carries specific assumptions about the nature of the data, the relationship of the researcher 
to the data, and the way analyzed data should be represented.» (Paterson et al., 2001, p. 115)

"Although we agreed that constructionism was the appropriate paradigm to underpin our qualitative meta-analysis, we were influenced by Schwandt's (2000) explication of "weak" versus "strong" constructionism. We therefore chose as our theoretical foundation weak constructionism, a perspective that rejects the nihilistic normative stance of the more radically relativistic epistemologies (pp. 198-199). Schwandt stated that strong constructionism can lead to "epistemological relativism" wherein knowledge claims and meanings are interpretable from within only a particular conceptual scheme (p. 200). Because strong constructionism holds that different paradigms are incommensurable, this theoretical framework would seem to preclude translations across studies, and for this reason we rejected it. With these understandings, we set out to determine the range of possibilities that existed for combining qualitative data to create new understandings.» (McCormick et al., 2003, p. 937)

«Meta-method offers a strategy by which to reflect on the role of methodological orientation and decision making as it shapes the findings of individual studies, and it also provides an angle from which to interpret a body of knowledge as it has evolved. From meta-method analysis, we begin (a) to identify the nuances in the various qualitative approaches that have been applied and the patterns in how and why such approaches may have been used in contrast with others and (b) to consider in a more abstract sense the kinds of knowledge that may not have been included in currently held conceptualizations as a result of these methodological choices. To some extent, method reflects academic discipline, in that certain disciplines have long been associated with the development and ownership of certain methodological traditions, but to an extensive degree, the original role of disciplinary orientation has been blurred as qualitative health researchers have adopted, adapted, and refined their approaches to answering research questions (Thorne, 1991). Therefore, the method, even more than the theoretical or disciplinary orientation, can often 
account for what we think we know about a field through analysis of the available body of qualitative research reports. [...]

The process of exploring and analyzing the impact of assumptions underlying data analysis requires a fairly intimate familiarity with the epistemological and ontological foundations of many approaches to data analysis. In the meta-synthesis phase of a metastudy, the intent is not to judge the relative merits of the various analytic options, but rather to try to appreciate how they may have influenced the nature of the research findings. For example, whereas some analytic methods capitalize on capturing and accounting for intricate variations within original data, others focus on collapsing variations into an increasingly smaller number of researcher-identified codes and categories. In some cases, this kind of process disembodies the data from the person who produced them and the context in which they were derived (Conrad, 1990). Thus, meta-method allows the metasynthesist to consider the degree to which the context and language of the primary research participants may have been sacrificed to expedite categorizing the data and whether this may have implications for the body of knowledge that has ensued. [...]

Thus, meta-method contributes to a meta-synthesis in creating the framework within which different discoveries about a phenomenon can be understood and contextualized.» (Paterson et al., 2001, p. 115-117)

\section{6. Étapes et procédures}

Pour cette catégorie, nous avons regroupé les rubriques «Étapes» et «Procédure» de la grille d'analyse, car il était plutôt difficile de démêler l'un de l'autre, alors même que nous trouvions peu d'extraits explicitant clairement une manière structurée d'effectuer une métasynthèse. Il y a, encore une fois, quelques éléments d'informations contenus dans les extraits inclus dans les catégories «Définitions» et «But». 


\subsection{Extraits de textes}

(Métathéorie) «1. Identifying the major cognitive paradigms that underlie the theory; 2. Identifying the assumptions underlying the theory; 3. Examining the historical evolution of the theory, including how it has changed over time, adaptions to its original conception, and significant landmarks in its evolution; 4. Determining how the sociocultural, disciplinary, and political context may have influenced the selection of theorical frameworks or their interpretation or research findings to support a particular theory; 5. Evaluating the quality of the selected theory.» (Paterson et al., 2001, p. 96)

«From my perspective, metasynthesis is a study unto itself that involves a systematic approach (Jensen \& Allen, 1996). Thus, there are scientific rules to follow and guidelines to assess achievement. There are rules about how to choose studies, how to resolve contradictory findings, how to reduce reviewer bias, how to summarize findings, how to integrate and interpret findings, and how to synthesize findings. A specific problem is defined; inclusion criteria are explicated; a retrieval process is identified; characteristics of the study are measured on a common scale; findings are identified, classified, and coded; findings are aggregated; and indices of effect magnitude are calculated and, ultimately, transformed into a new conceptualization. The key characteristic of a metasynthesis is the transformation of this new conceptualization. Because of this, it is clearly not an approach to be undertaken by the novice.» (Jensen in Thorne et al., 2004, p. 1346)

«[...] deconstructed, reconstructed, and synthesized report findings accross studies to better explicate the concept of courage [...].» (Finfgeld, 2003, p. 897)

«In the case of theory building, findings from a numberof studies are used to "push the level of theory" beyond what is possible in a single investigation (Schreiber et al., 1997, p. 315)» (Finfgeld, 2003, p. 894-895) 
"An identifiable difference among data analysis methods is the degree to which researcher deconstruct and decontextualize the original study findings prior to drawing inferences. [...] In these instances, the data analysis process begins with the recoding of findings from each study and gradually moves toward the synthesis and translation of findings across studies. Conversely, investigators might begin with a list codes and examine each study in isolation before synthesizing the coded data (e.g., McNaughton, 2000). Finally, others do little reanalyzing of the original study findings. Instead, they focus on synthesizing and translating the central study metaphors from the outset (e.g., Paterson, 2001). (Finfgeld, 2003, p. 900)

«Following data retrieval, the process of interpretive synthesis begins. The interpretive synthesis process has two aspects: hermeneutic and dialectic. The hermeneutic aspect consists of portraying individual constructions accurately, whereas the dialectic aspect consists of comparing and contrasting these individual constructions. The aim is one of generating a new construction on which there is consensus. First, the texts are compared in a holistic interpretation with the synthesis taking the form of reciprocal translations, that is, each study is translated into the terms (metaphors) of the others and vice versa (Noblit $\&$ Hare, 1988). In the initial phase of reading the texts, the findings are standardized via common codes, outlines, and reporting formats. To ensure the inclusion of all relevant findings, extensive attention should be given to the details in the individual accounts, and what was said about the substantive concerns of the phenomenon. Next, the various studies within each group are put together, determining the relationship between the studies to be synthesized. At this time, initial assumptions about the relationships between studies are made. A list of key metaphors, phrases, concepts, ideas, and/or categories used in each account are created and juxtaposed. The next phase involves translating the studies into one another. This translation maintains the central metaphors of each account in relation to key metaphors in the other accounts. The last phase involves synthesizing these translations, making the clustered meta-matrices progressively more refined, leading to a description of the phenomenon.» (Jensen et Allen, 1996, p. 555-556) 


\section{Exemples}

Pour cette catégorie de la grille d'analyse, nous n'avons pas trouvé ce que nous cherchions, ou plutôt, il n'est pas possible d'exprimer ici que nous voulions trouver, soit de très courts résumés de style «Problème-méthode-résultats» démontrant l'utilisation de la métasynthèse et de la méta-analyse qualitative. Cependant, nous mettons les références bibliographiques de quelques études qui sont des métasynthèses de même que des métaanalyses qualitatives et auxquelles nous allons nous référer pour illustrer comment ces méthodes peuvent s'articuler de façon concrète.

\subsection{Exemples de méta-analyses qualitatives}

Chen, T-Y. et Turner, S. (s.d.). A qualitative meta-analysis on web-based learning in higher education : a grounded theory approach.

Consulté le 1 décembre 2006

[http ://oak.cats.ohiou.edu/ chent/RCET-GrantProposal-0903-2001.pdf]

Dewitt-Brinks, D. et Rhodes, S.C., (1992). Listening instruction : a qualitative metaanalysis of twenty-four selcted studies. Présenté au Annual meeting of the International communication association (Miami, FL).

Feder, G.S., Hutson, M., Ramsay, J. et Taket, A.R. (2006). Women exposed to intimate partner violence. Arch intern med, (166), 22-37.

Yu, S-H., Kim, C-B., Park, J.W., Kim, M.S. et Radosevich, D.M. (2005). Ultrasonography in the diagnosis of appendicitis : evaluation by meta-analysis. Korean journal of radiology, (6)4, 267-277. 


\subsection{Exemples de métasynthèses}

Coffey, J. S. (2006). Parenting a child with chronic illness : a metasynthesis. Pediatric nursing. (32)1, 51-59.

Kärkkäinen, O., Bondas, T. et Eriksson, K. (2005). Docimentation of individualized patient care : a qualitative metasynthesis. Nursing ethics, (12)1, 123-131.

McCormick, J., Rodney, P. et Varcoe, C. (2003). Reinterpretation accross studies : an approach to meta-analysis. Qualitative health research, (13)7, 933-944.

Tellez, K. et Waxmann, H.C (s.d). A meta-synthesis of qualitative research on effective teaching practice for English language Learners. In Norris, J.M., Ortega, L. (dir.). Synthesizing research on language learning and teaching. Philadelphia : John Benjamins Publishing. Consulté le 19 janvier 2006.

[http ://education.ucsc.edu/faculty/ktellez/tel-wax-metasynth.pdf]

\section{Limites}

Comme toutes les méthodes de recherche, la métasynthèse, la méta-analyse qualitative et la méta-étude possèdent des limites. Nous avons décelé des passages qui en faisaient mention et nous les avons répertoriés dans l'optique de proposer des portraits aussi valides que possible de chacune de ces approches.

\subsection{Extraits de textes}

«First, most qualitative meta-analysis techniques are relatively new and remain poorly developped.» (McCormick et al., 2003, p. 934) 
«Metasynthesis data analysis methods are far from uniform. For instance, some researchers object to interpreting findings resulting from different epistemological perspectives because of their variant foci and theoretical structures (Estabrooks et al., 1994, Jensen et Allen, 1996) [...] In contrast, some investigators have found this restriction unnessary, and, in fact, they embrace the opportunity to synthesize studies from differing epistemological perspectives.» (Finfgeld, 2003, p. 900)

«Critics caution that combining results from differing epistemological perspectives can be difficult (Estabrooks et al., 1994) and could yield misrepresentations of the original research findings (Jensen et Allen, 1996). (Finfgeld, 2003, p. 902)

«As we discovered, the more thoroughly you have examined the methodological, disciplinary, and theoretical underbelly of the existing body of knowledge about a phenomenon, the more difficult it can become to make definitive claims about its nature (Thorne, 2001).» (Thorne in Thorne et al., 2004, p. 1357)

«Several problems have been identified which must be considered in the conduct of meta-analytic procedures. These include: reliability of data retrieval, missing data, sampling bias, loss of information, glossing over details, heterogeneity of method, heterogeneity of quality, differing levels of analysis, deductive contamination from other theories/literature, and exaggeration of descriptions and interpretations-pushing beyond credibility. As meta-syntheses of qualitative accounts are beginning to be conducted (Field \&Marck, 1994; Jensen \& Allen, 1994; Morse \& Johnson, 1991), there is a need for guidelines concerning the method. These seem essential for dealing with issues involved in the application of meta-analytic procedures for qualitative accounts.» (Jensen et Allen, 1996, p. 556) 


\section{Forces}

Au même titre que pour les limites, chaque méthode possède des points forts et comme il a été mentionné dans la catégorie «Pertinence», la présente catégorie de la grille d'analyse se combine naturellement à d'autres pour exprimer l'utilité de la métasynthèse comme méthode de recherche.

\subsection{Extraits de textes}

«[...]small gains can be as important as larger ones and that better ways of theorizing about narrow aspects of a field may be more useful in the long run than are completely original grand theories.» (Paterson et al., 2001, p. 120-121)

«Thus, credibility has the potential to be enhanced when new translations are metasynthesized from findings that have been generated from differing philosophical and methodological perspectives.) (Finfgeld, 2003, p. 902)

"We take the position that, rather than providing a means to greater "truth," qualitative meta-analysis is another "reading" of data, an opportunity to reflect on the data in new ways. It does not necessarily provide more accurate or truthful accounts by virtue of having more data, collected by multiple researchers in different settings. Nevertheless, there are significant advantages in being able to draw on qualitative data across a number of studies that, we believe, cannot be realized in single studies. In this, we agree with Sandelowski and colleagues (1997), who argued that although the notion of generalization as it is conceived of in the quantitative tradition is not appropriate for qualitative work, naturalistic or ideographic generalizations -generalizations about particulars- can and should be made across qualitative studies. Sandelowski et al. maintained that such efforts help avoid a failure of qualitative researchers to go far enough in their analyses. We were seeking an approach that allowed us to draw on a broader and richer data set to answer new 
questions. Our aims were to represent our individual work in light of each other's work in ways that demonstrate both the convergences and the differences across our studies to create "more informed and sophisticated reconstructions" (Lincoln \& Cuba, 2000, p. 166) as a basis for praxis and for influencing policy toward health and social justice.» (McCormick et al., 2003, p. 936)

«In the final analysis, therefore, some of us concluded that metastudy tends to serve us far better as a method for rigorously and systematically deconstructing existing bodies of qualitative research findings than it does as a technique for synthesizing powerful new products (Thorne, Paterson, et al., 2002).» (Thorne in Thorne et al., 2004, p. 1357)

Cette section avait comme objectif de présenter le contenu manifeste issu de l'analyse de contenu des divers textes répertoriés traitant de la métasynthèse, de la métaanalyse qualitative et de la méta-étude. Ce ne sont pas tous les textes qui ont fourni des informations pertinentes ou utiles, mais le cas échéant, nous les avons présentés ici. Les extraits dégagés ont permis de faire la rédaction d'un article qui présente les résultats de la recherche. Cet article, l'objet du prochain chapitre poursuit le but de mieux comprendre la métasynthèse et de la différencier de la méta-analyse qualitative.

\section{LA STRATÉGIE DE PRÉSENTATION DES RÉSULTATS}

Les résultats des analyses de contenu effectuées sont présentés à l'intérieur d'un article scientifique. Tout d'abord, une section décrit sommairement la méta-analyse qualitative. Dans cette section, nous nous penchons sur les éléments ressortis des recherches analysées pour mettre en évidence les caractéristiques propres à la méta-analyse qualitative, similaires ou distinctes de la métasynthèse. Une fois cet exercice complété, nous présentons de façon plus détaillée la métasynthèse. Dans un but de l'efficacité de l'argumentation, nous combinons en quelques grandes sections l'ensemble des thèmes de la grille d'analyse. Ainsi, au départ, nous tentons d'établir une définition globale de la métasynthèse qui rejoint la vision de la plupart des auteurs étudiés sur le sujet. Nous 
examinons également comment différents chercheurs ont appliqué la métasynthèse et d'où vient ce type de méthode. Une fois cette étape cruciale faite, nous expliquons ensuite les buts de la métasynthèse, les raisons de son utilisation, la pertinence de cette méthode. Par la suite, dans l'optique de bien comprendre tout le fonctionnement de la métasynthèse, nous explicitons ses composantes et des façons de procéder pour sa mise en oeuvre. Avant de conclure, nous revenons sur des éléments conceptuels majeurs pour comprendre diverses perspectives de la métasynthèse. En conclusion, nous exposons certaines limites de cette méthode, ce qui termine le portrait de la métasynthèse. Le résultat final se veut un texte aussi complet que possible sur les grands principes de la métasynthèse, de ses objectifs à son utilisation. 


\section{CHAPITRE QUATRE \\ LA DISCUSSION}

Le dernier chapitre de ce mémoire par article contient l'article qui a été soumis à la revue Recherches qualitatives. Les rôles des coauteurs sont indiqués à l'Annexe F, l'accusé de réception de la part de l'éditrice de la revue à l'Annexe $G$ et les normes de publications de la revue à l'Annexe $\mathrm{H}$. Avant une éventuellement parution, celui-ci sera évalué tout d'abord par l'équipe éditoriale et ensuite à l'aveugle par au moins deux arbitres.

L'article a été soumis à la revue selon ses normes. Dans le but d'en faciliter la lecture à l'intérieur de ce mémoire, l'interligne et les marges ont été ajustés pour rencontrer les normes de présentation de la Faculté d'éducation de l'Université de Sherbrooke. Cela a aussi eu pour effet de réduire de façon substantielle le nombre de pages. Notez également que les notes de fin de document présentes dans l'article soumis ont été transformées en notes de bas de pages pour des questions techniques et de présentation. Cependant, sur la forme générale et le contenu, le texte présenté ici est fidèle à l'article reçu par la revue.

\section{Étude comparative de la métasynthèse et de la méta-analyse qualitative}

\section{RÉSUMÉ}

Cet article fait le point sur une méthode d'analyse secondaire de recherches qualitatives, la métasynthèse. Pour ce faire, nous nous penchons sur les écrits de plusieurs auteurs et nous tentons d'établir des consensus, par exemple, sur sa définition, ses buts, ses composantes, ses limites. Avant d'entrer dans le coeur de la métasynthèse, nous abordons brièvement la méta-analyse qualitative, une méthode d'analyse secondaire plus connue et plus utilisée. Cela permet de mettre en relief les approches d'analyse secondaire en qualitatif et surtout de dégager les différences entre la méta-analyse qualitative et la 
métasynthèse. Ensuite, l'analyse des textes sur le sujet permet de décrire la métasynthèse. Il ressort que ses finalités sont de produire une nouvelle compréhension ou une théorie nouvelle par rapport à un phénomène. Le consensus n'est cependant pas atteint chez les auteurs sur le potentiel de la métasynthèse, de plus amples réflexions et écrits se révélant nécessaires.

Mots-clés: métasynthèse, métaétude, méta-analyse qualitative, synthèse

\section{INTRODUCTION}

En recherche, spécialement dans le grand champ des sciences sociales, explorer ou comprendre un phénomène relève souvent d'une étude basée sur l'approche qualitative. Comparativement à l'approche quantitative qui verra davantage à démontrer des liens de cause à effet entre diverses variables, l'approche qualitative se veut plus compréhensive et souvent plus créative dans ses méthodes de cueillette de données et d'analyse de celles-ci. Cela a plusieurs effets positifs, mais plusieurs lui reprocheront, à tort ou à raison, un manque de rigueur, ou encore l'impossibilité de généraliser les résultats des recherches. Malgré certaines réserves, il existe des façons de réanalyser des études qualitatives pour en arriver à une synthèse, à une certaine généralisation, ou encore à une compréhension nouvelle d'un phénomène donné. Pour ce faire, il existe plusieurs méthodes d'analyse secondaire de recherches qualitatives tout comme il en existe plusieurs du côté quantitatif de la recherche. Dans un article, Synthesising qualitative and quantitative evidence : a review of possible methods, Dixon-Woods, Agarwal, Jones, Young et Sutton (2005) recensent onze méthodes reconnues dans les approches quantitatives et qualitatives, une des méthodes les plus connues étant sans doute la méta-analyse quantitative. Or, même si ce genre de méthodes existe en qualitatif, leur présence est plus récente, leur pertinence moins reconnue et leur utilisation moins répandue. Conséquemment, l'expertise méthodologique est moins développée dans ce champ que l'on pourrait encore qualifier de relativement nouveau. Il existe ainsi du flou à cet égard, plusieurs incompréhensions mises 
en évidence par les chercheurs qui les utilisent et plusieurs mises au point sont à faire, comme le soulignent Dixon-Woods et al.

Pour bien comprendre les limites des méthodes d'analyse secondaire, il s'avère utile de revenir dans le temps. Sans effectuer un long retour et une analyse historique de la recherche scientifique qualitative, il demeure toujours pertinent de pouvoir situer, d'être en mesure de contextualiser l'émergence de méthodes de recherche. Dans le cas présent, pour déterminer la provenance des méthodes d'analyse secondaire de recherches qualitatives, il est nécessaire de voir plus large et de commencer par les différentes formes de métarecherches. Un bref retour est effectué sur ce sujet par Paterson, Thorne, Canam et Jillings (2001) dans le chapitre d'introduction de Meta-study of qualitative health research - a practical guide to meta-analysis and meta-synthesis. En se référant à Alexander et Colomy (1992), de même qu'à Ritzer (1992), ils expliquent qu'au départ, ce sont des « chercheurs postpositivistes qui ont exprimé un intérêt considérable dans la synthèse de diverses positions théoriques et disciplinaires en de grandes théories. $)^{26}$ (p.1, traduction libre) En sociologie, la métasociologie fit son apparition voilà plus de 50 ans, pour plus tard être modifiée en métathéorisation. En anthropologie, Noblit et Hare marquèrent le domaine en développant la méta-ethnographie vers la fin des années 1980. Au début des années 1990, Zhao explicita la métaétude, une autre approche, assez structurée, qui a pour but de jeter un regard nouveau sur des études primaires. Environ dix ans plus tôt, on commençait à parler de méta-analyse quantitative, une approche présentée par Glass, Smith et McGraw qui s'avéra très populaire. C'est cependant en 1985 que Stern et Harris présentèrent une variante de cette méta-analyse, la version qualitative. Bref, le survol de Paterson et al. montre que même si plusieurs chercheurs ont effectué, ou tenté d'effectuer, des synthèses d'études qualitatives auparavant, c'est réellement à partir de 1994 que des efforts furent entrepris afin de clarifier ce champ. En effet, les chercheurs ont commencé à s'interroger davantage et à vouloir clarifier les méthodologies utilisées dans ce genre d'études, de même qu'à instaurer un langage plus uniforme concernant les synthèses en

\footnotetext{
${ }^{26}[\ldots]$ post-positivists scholars expressed considerable interest in synthesizing diverse theorical and disciplinary positions into grand theories.
} 
qualitatif (Finfgeld, 2003). Pourtant, comme nous le constaterons, ce ménage conceptuel n'est pas encore complété. C'est dans cette optique que nous abordons tout d'abord la métaanalyse qualitative pour mettre en contexte les méthodes d'analyse secondaire de recherches qualitatives pour ensuite présenter un portrait de la métasynthèse, aussi fouillé que possible.

\section{MÉTHODOLOGIE}

Pour écrire cet article, nous avons effectué une analyse de contenu sur une série de documents. Certains portent sur la métasynthèse, la méta-analyse qualitative ou la métaétude, alors que d'autres présentent des résultats de recherches basées sur l'une de ces méthodes. Nous avons cherché à déceler dans ces documents (des articles et un livre) des éléments d'information selon des thèmes pré-établis comme «Définitions», «Buts», «Limites», etc. Nous en avions 10 au départ que nous avons finalement regroupé pour en arriver, dans le cas de la métasynthèse, à quelques grandes caégories présentées plus bas. Concernant la méta-analyse qualitative, nous avons tout regroupé dans la section qui suit.

\section{LA MÉTA-ANALYSE QUALITATIVE}

La méta-analyse qualitative (MAQ1) est généralement acceptée comme étant une analyse secondaire qui a comme origine la méta-analyse quantitative. À l'instar de cette dernière, la MAQl a comme objectif principal de regrouper ensemble un grand nombre d'études primaires pour en combiner les résultats et ainsi obtenir une représentation plus large d'un phénomène donné. Dans Ultrasonography in the diagnosis of appendicitis : evaluation by meta-analysis, Yu, Kim, Park, Kim et Radosevich (2005) écrivent de la méta-analyse qu'il s'agit « de la revue critique et de la combinaison évaluative statistique des résultats de recherches antérieures. ${ }^{27}$ (p. 268, traduction libre) Cette définition assez

\footnotetext{
${ }^{27}$ Meta-analysis is the critical review and statistical combination and evaluation of the results of previous research [...].
} 
large convient très bien à la méta-analyse quantitative, mais pas complètement à la MAQ1, essentiellement à cause de l'aspect statistique, bien que la revue critique et la combinaison évaluative soient des points pertinents. D'ailleurs, d'autres chercheurs vont emprunter des approches résolument qualitatives, comme en fait foi le titre suivant d'un article : $A$ qualitative meta-analysis on web-based distance learning in higher education : a grounded theory approach (Chen et Turner, 2001). Dans la cadre de la MAQl réalisée dans cette étude, les auteurs se sont basés sur la théorie ancrée. L'un des objectifs de l'étude rejoint ce que nous énoncions plus tôt, soit le regroupement d'études : « résumer et synthétiser les études existantes portant sur l'apprentissage à distance dans le but de comprendre ce qui fonctionne et ce qui ne fonctionne pas dans les programmes d'apprentissage à distance aux études supérieures [...]. $\|^{28}$ (p. 2, traduction libre)

Ce concept de regroupement d'éléments dégagés d'études primaires se retrouve également dans une étude intitulée Women exposed to intimate partner violence. Feder, Hutson, Ramsay et Taket (2006) utilisent la MAQI dans une optique proche de celle de Chen et Turner, pour faire ressortir certains concepts clés des études primaires analysées. Dans une perspective davantage qualitative, ils cherchent tout d'abord des construits de premier niveau, lesquels sont la compréhension de la personne (l'intervieweur) qui rapporte les propos des répondants (dans ce cas-ci, l'expérience vécue en milieu de la santé par des victimes de violence conjugale) pour ensuite les comparer avec les construits de deuxième niveau, soit les conclusions des chercheurs de ces études primaires. Ce qui résulte de ces comparaisons (positives, négatives, intraétudes, interétudes, etc.) est nommé construit de troisième niveau. Dans ce cas-ci, nous sommes à même de constater qu'il y a eu un processus d'interprétation des résultats trouvés. Mais ce n'est pas toujours le cas avec la MAQl, comme en fait foi l'étude Listening instruction : a qualitative metaanalysis of twenty-four selected studies. Dans cette recherche, Dewitt-Brinks et Rhodes (1992) ont voulu trouver ce qui aidait ou nuisait à l'apprentissage de l'écoute. Pour ce faire, les textes choisis ont été analysés selon des critères prédéfinis et des extraits de textes

\footnotetext{
${ }^{28}$ [...] summarize and synthesize the existing distance learning studies in order to understand what works and what does not in higher education distance learning programs.
} 
pertinents aux critères ont été compilés, sans grande interprétation. Ici, la MAQl se rapproche davantage de la revue de littérature. Pour d'autres chercheurs tels Yu et al., la MAQl semble plutôt être une étape préliminaire de sélection d'études primaires dans le but de faire ultérieurement une méta-analyse quantitative. En fait, dans la recherche de Yu et $a l$., la MAQI sert à identifier, à l'aide d'un processus systématique, la qualité d'ensemble d'une multitude d'études présélectionnées pour finalement garder celles qui répondent aux normes déterminées par l'équipe de recherche. Cette dernière approche paraît cependant être marginale et ne pas refléter l'usage généralisé de la MAQ1.

Il existe une autre interprétation de la MAQ1 à prendre en considération : certains auteurs vont utiliser le terme "méta-analyse qualitative" pour se référer à un ensemble de méthodes d'analyse secondaire, pas simplement à une méthode bien définie, complète en elle-même. Par exemple, en lisant l'article de McCormick, Rodney et Varcoe (2003), Reinterpretation across studies : an approach to meta-analysis, il est vite évident que les auteures parlent de la MAQl dans le sens d'une famille et non pas dans le sens d'une méthode. À preuve, lorsqu'elles énoncent une série de considérations concernant la MAQl, elles écrivent : " Premièrement, la plupart des techniques de méta-analyse qualitative sont relativement nouvelles et demeurent peu développées. $»^{29}$ (p. 934, traduction libre) D'ailleurs, elles écriront souvent qualitative meta-analyses, au pluriel, se référant à un ensemble d'approches. Cette vision de la MAQl n'est cependant pas partagée par tous, mais elle existe et nous verrons un peu le même phénomène avec la métasynthèse.

En résumé, la MAQl est une méthode qui présente assurément des avantages, ne serait-ce que pour obtenir une vue d'ensemble d'un phénomène donné. Par contre, cette vue d'ensemble est souvent le résultat d'une simple accumulation de résultats. Bien sûr, cet exercice est fait de façon rigoureuse et les résultats sont tout autant valides que le seraient ceux d'une autre recherche. Par contre, il semble manquer à la MAQ1 un côté réflexif, une analyse plus profonde des études primaires prises en considération. D'ailleurs, c'est presque

\footnotetext{
${ }^{29}$ First, most qualitative meta-analysis techniques are relatively new and remain poorly developped.
} 
toujours le terme agglomeration qui est utilisé pour décrire la MAQI. À l'opposé, presque tous les auteurs se feront un point d'honneur d'écrire que la métasynthèse « is not a simple agglomeration ».

\section{LA MÉTASYNTHÈSE}

Le très bref survol de la méta-analyse qualitative nous a permis de dégager ce qui peut être fait avec une approche d'analyse secondaire. Bien qu'encore marginale, la MAQ1 demeure probablement une de ces approches les plus connues en son genre. Parmi celles-ci se trouve la métasynthèse, présente presque exclusivement dans la littérature méthodologique des sciences infirmières. Est-elle restreinte à ce domaine? En pratique actuelle, il semble que oui. Mais nous ne voyons pas pourquoi il devrait en demeurer ainsi, des chercheurs ayant par exemple tissé des liens évidents entre la recherche qui se fait en sciences infirmières auprès des patients et des soins promulgués et, pour n'en nommer que deux ou trois, les domaines de l'éducation, du travail social ou de l'anthropologie (Bernard, 2000). C'est dans cette optique que nous jugeons pertinent d'approfondir les connaissances sur la métasynthèse, plusieurs domaines de recherche pouvant éventuellement en bénéficier.

\section{Qu'est-ce que la métasynthèse?}

À étudier la métasynthèse, on se rend vite compte que mettre en commun l'information qui la concerne, sortir les tendances et en arriver à une définition consensuelle - probablement l'élément le plus important pour sa compréhension - s'avère finalement être une entreprise assez fastidieuse. Pour ce faire, il faut se référer à l'interprétation qu'en font plusieurs auteurs, car il n'existe pas, pour imager, d'entrée intitulée « métasynthèse » dans les dictionnaires ou encyclopédies. Même les ouvrages généraux traitant de la recherche qualitative, ou encore de la méthodologie de la recherche, ne s'y intéressent pas. Il existe cependant quelques articles sur le sujet, et c'est ce sur quoi nous nous basons pour 
comprendre la métasynthèse. Ainsi, dans un excellent article-synthèse sur le sujet, Metasynthesis : the state of the art - so far, Finfgeld (2003) dresse un portrait interressant de cette méthode. Elle propose une définition se lisant ainsi : « terme "parapluie" se référant à la synthèse des résultats de plusieurs recherches qualitatives pour en créer une nouvelle interprétation. ${ }^{30}$ (p. 895, traduction libre) Il s'agit d'une description assez générale, mais qui reflète bien l'idée de base de cette approche. En citant d'autres auteurs, Finfgeld donne de plus amples détails, conduisant à une définition, à une description, plus poussées. Ainsi :

" la métasynthèse ne serait pas une revue systématique de la littérature (Schreider et al., 1997; Sherwood, 1999) ou encore le réarrangement de résultats de recherche (Sherwood, 1999). Il ne s'agit pas non plus de l'addition des constats de diverses études, ou encore d'une analyse conceptuelle. Plutôt, "il s'agit de mettre ensemble et de décomposer des résultats, les examiner, découvrir les éléments fondamentaux et, en quelque sorte, combiner divers phénomènes dans un tout transformé." (Schreider et al., 1997) ${ }^{31}$ (idem, traduction libre)

Cette compréhension du concept de la métasynthèse, Louise Jensen (2004) l'exprime clairement. Dans un article, Qualitative metasynthesis : reflections on methodological orientations and ideological agenda, Jensen explique tout d'abord elle aussi qu'une métasynthèse se doit d'être autre chose qu'une simple accumulation de données. Il ne s'agit pas de chercher à atteindre impérativement un consensus final unanime, d'autant plus que ses finalités sont davantage de l'ordre de l'interprétation, devant prendre en compte les différences qui émanent des études analysées. Un paragraphe de Jensen révèle très bien diverses caractéristiques de la métasynthèse qui permettent d'en avoir une compréhension plus approfondie :

"La métasynthèse est une méthode fondamentalement conçue pour faciliter le développement des connaissances. En ce sens, elle peut être vue comme étant une forme de discours qui contribue à une compréhension toujours plus poussée du phénomène d'intérêt. Les particularités sont retenues et la reconstruction [du phénomène] amplifie la complexité. [La métasynthèse] pourrait donc être

\footnotetext{
${ }^{30}$ Umbrella term referring to the synthesis of findings across multiple qualitative reports to create a new interpretation.

${ }^{31}$ Metasynthesis is neither a systematic review of the literature [...] nor the collating (i.e., codifying) of research findings [...]. In addition, it is not the aggregation (i.e., summing) of research outcomes or a concept analysis. Rather, "it is the bringing together and breaking down of findings, examining them, discovering the essential features, and, in some way, combining phenomena into a transformed whole[...].
} 
considérée comme une déconstruction avec l'objectif de restructurer un ordre à partir duquel on travaillera. Selon cette approche, la métasynthèse se veut une traduction, une façon de comprendre des particularités à l'intérieur d'un tout. Appliquée à un corps de recherche, cela réduit, sans éliminer, l'incertitude ${ }^{32}$ (p. 1346; traduction libre)

Jensen demeure par ailleurs fidèle à un article qu'elle a coécrit en 1996 avec Marion N. Allen, Meta-synthesis of qualitative findings, dans lequel elles considèrent que « [la] métasynthèse diffère d'une simple logique d'accumulation ou de normalisation [averaging] effectuée sur plusieurs études. La singularité ou l'holisme des comptes-rendus est conservé malgré le fait d'être synthétisés lors du transcodage; les textes sont comparés pour créer une interprétation holistique. ${ }^{33}$ (p. 554, traduction libre) D'autres auteurs confirment et ajoutent des points à ces définitions. Ainsi, Sandelowski et Barroso (2003), dans Writing the proposal for a qualitative research methodology project, expriment que la métasynthèse, dans sa spécificité, rejoint quelque peu la méta-analyse quantitative dans la mesure où les deux approches veulent se baser sur des analyses secondaires structurées pour combiner des résultats scientifiques. Le rapprochement entre la métasynthèse et la méta-analyse quantitative s'arrête cependant là. Par ailleurs, il est intéressant de noter que les auteures mettent en évidence une différence entre la métasynthèse et d'autres approches qualitatives. Elles considèrent qu'il existe diverses approches de synthèse en recherche qualitative, et que la métasynthèse est, ou se doit d'être, par nature, plus structurée.

Pour poursuivre, demeurons avec Margarete Sandelowski, une auteure-chercheuse de renom concernant la métasynthèse. Plusieurs écrits sont le fruit de travaux de sa part et plusieurs articles sur cette méthode se réfèrent à elle, entre autres dans le cas de chercheurs qui ont fait des métasynthèses - par exemple, Kärkkäinen, Bondas et Eriksson (2005);

\footnotetext{
${ }^{32}$ Metasynthesis is a method inherently designed to facilitate knowledge development. As such, it can be understodd as a form of discourse that contributes to a fuller understanding of the phenomenon of interest. Particularities are retained, and the reconstruction enhences the complexity. It might tehrefore be considered a deconstruction for the purpose of restructuring an order from which to operate. In this way, metasynthesis functions as a translation, a means to grasp the particulars within the wholes. Applied to a body of research, it reduces, but does not eliminate, uncertainty.

${ }^{33}$ This meta-synthesis differs from the simple accumulative logic or averaging accross studies. The uniqueness and holism of accounts is retained even though synthesized in the translation; texts are compared to create a holistic interpretation.
} 
Téllez et Waxman (s.d.); McCormick, Rodney et Varcoe (2003); Coffey (2006). Dans Qualitative metasynthesis : reflections on methodological orientation and ideological agenda, un écrit collectif, Sandelowski (2004) reprend des propos écrits dans un texte antérieur et explique que la métasynthèse, tout en étant une technique d'intégration de résultats qualitatifs, interprète ces résultats pour en arriver à des conclusions. Cependant, la rigueur devient ici un enjeu dans la validité de la recherche, car les résultats intégrés sont eux-mêmes des interprétations de cas, de phénomènes, ou encore d'événements tirés d'études phénoménologiques, d'études ethnographiques, de théories ancrées et d'études explicatives ou descriptives. Le concept d'interprétation est important dans le cas de la métasynthèse, car il fait opposition à l'addition simpliste de données. Ainsi, ce qui est recherché, c'est essentiellement une nouvelle interprétation offrant une description ou encore une explication propre à un phénomène particulier. Dans la même veine, McCormick, Rodney et Varcoe (2003) ont réfléchi sur ce qu'ils appellent la méta-analyse qualitative (mais qui est en réalité davantage une métasynthèse) pour conclure, après cinq ans de travaux, que le but d'une telle approche n'est pas d'en arriver à une "vérité », mais de voir le processus comme une façon différente de lire des données, profitant du fait de pouvoir se baser sur plusieurs études. Par ailleurs, ils considèrent que la généralisation n'est pas le but ultime, mais que la possibilité existe, d'autant plus qu'une somme importante de travail a été investie dont une grande attention à demeurer fidèles aux propos originaux. Il est à noter que les généralisations faites dans ce cas seraient plutôt du genre naturaliste ou encore idéographique, c'est-à-dire conformes aux conditions particulières des études primaires. D'un point de vue plus théorique, ces auteurs apportent un élément intéressant en rapport avec le paradigme où se situe la métasynthèse. Selon eux, elle devrait se retrouver dans le paradigme constructiviste, plus spécifiquement dans du constructivisme faible, versus fort. Ainsi, on évite d'avoir affaire avec du relativisme épistémologique qui empêche d'exporter des résultats à d'autres contextes. Le constructivisme fort rendrait difficile la combinaison d'études primaires, celui-ci statuant sur l'incompatibilité de paradigmes différents. Mais, parce que le constructivisme permet la reconstruction d'un savoir à l'aide de données de plusieurs études et que sa variante faible ne présente pas les rigidités de sa variante forte, c'est dans ce paradigme que McCormick et al. ont décidé 
d'inscrire leur approche méta-analytique. Paterson et al. (2001) acceptent elles aussi la possibilité d'inscrire la métasynthèse dans le paradigme constructiviste, surtout dans une perspective de métaétude, mais elles mettent un bémol. En effet, elles reconnaissent dans le constructivisme l'aspect de construction sociale qui sied bien à la métasynthèse, mais dans un contexte plus large que le simple ajout de connaissances au savoir actuel d'un phénomène. De plus, pour la recherche en sciences de la santé où la métasynthèse est profitable, le paradigme du réalisme modéré est également soutenu par les auteures. Nous verrons que d'autres paradigmes semblent également convenir à la métasynthèse.

À partir des éléments descriptifs présentés, il se dégage que la métasynthèse ne peut pas être qu'une simple addition de résultats, qu'il doit y avoir une transformation de ces résultats, une réinterprétation qui permettra de pousser plus loin la compréhension du

phénomène étudié. À cet égard, la métasynthèse se démarque de la méta-analyse qualitative par son orientation de réinterprétation de résultats d'études qualitatives. Malgré la possibilité d'offrir une vision généralisée d'un phénomène (but similaire à celui recherché par la méta-analyse), la métasynthèse peut très bien se limiter à revisiter un phénomène pour en offrir une interprétation nouvelle, ce qui peut mener en retour à d'éventuels avancements plus concrets.

\section{Pourquoi utiliser la métasynthèse?}

Comme nous venons de le voir, la métasynthèse n'est pas un concept encore clairement défini, certains points restent à clarifier. Malgré tout, il est possible de convenir de ses grandes orientations et particularités. Lorsqu'on explore les buts et les finalités attendues de l'utilisation de la métasynthèse, on examine le « pourquoi », la raison de faire une métasynthèse. Il faut donc être en mesure de bien comprendre les variantes de la métasynthèse pour être en mesure de l'employer à bon escient. Or, plusieurs caractéristiques demeurent globales et fidèles à la nature même de la métasynthèse. Quoiqu'il en soit, voyons quels buts un chercheur serait en mesure d'atteindre en effectuant une métasynthèse. 
En se référant à Sherwood (1999), Finfgeld (2003) explique que le but de la métasynthèse consiste à produire une interprétation nouvelle et intégrée des résultats des recherches analysées qui soit plus poussée, plus substantielle que les résultats de chacune des recherches prises individuellement. On cherche à clarifier des concepts et des modèles avec l'objectif de renchérir et solidifier l'état actuel des connaissances de même que les modèles et théories opérationnels qui sont en émergence. Jensen (2004), pour sa part, explique que "le résultat doit être quelque chose se rapprochant d'une compréhension commune de la nature d'un phénomène, non pas une vision du monde consensuelle. ${ }^{34}$ (p. 1346; traduction libre) Ailleurs, Jensen et Allen (1996) expliquent que, puisque la métasynthèse ne vise pas l'accumulation de données, mais plutôt leur intégration dans une nouvelle recherche, le but est de permettre la compréhension d'un phénomène ou l'apport d'explications interprétatives à son sujet. Le résultat ne devrait donc pas être l'émergence d'une nouvelle théorie prédictive en lien avec le phénomène étudié. Cela rejoint Sandelowski (2004) pour qui la métasynthèse ne signifie pas ultimement d'arriver à synthétiser ou à regrouper les résultats d'études, les méthodes de recherche et d'analyse ou les cadres de référence utilisés, mais plutôt de tendre vers le fait d'inventorier, de décrire, de comparer ou même de critiquer ces éléments. Sandelowski et Barroso (2003) expliquent que la métasynthèse devrait donner des résultats interprétatifs touchant la globalité des études primaires analysées, toujours en cherchant à demeurer fidèle aux résultats interprétatifs de ces mêmes études primaires. Pour renchérir, une conception intéressante de l'objectif de la métasynthèse se retrouve dans l'ouvrage de Paterson et al. (2001). Il s'agit de :

" creuser sous la surface de ce qui est présentement compris, de s'appuyer sur une analyse aussi complète que possible pour déconstruire la validité des idées qui ont cours présentement et de faire émerger les bases d'une nouvelle

\footnotetext{
${ }^{34}$ The outcome will be something like a common understanding of the nature of a phenomen, not a consensual wordview.
} 
"vérité", d'une meilleure compréhension, ou encore d'une approche plus socialement responsable de théoriser. ${ }^{35}$ (p. 111, traduction libre)

Ces mêmes auteures soutiennent également que la métasynthèse, dans un contexte de métaétude (concept sur lequel nous reviendrons), permet de questionner des théories existantes qui auraient tendance à presque toujours avantager certains groupes ou certaines situations au détriment d'autres, à cause d'une certaine position théorique, par exemple. De fait, la métasynthèse permet de tirer avantage de tous les points de vue, quoiqu'ils soient largement répandus ou non, et de réfléchir sur des faits et l'apport qu'ils peuvent faire au domaine.

Ce qui est proposé au paragraphe précédent montre qu'il existe une certaine cohésion par rapport à ce que la métasynthèse devrait permettre d'atteindre. Cependant, il existe des nuances dans le concept et dans la compréhension de ce qu'est la métasynthèse. Conséquemment, de multiples buts, parfois différents, mais parfois complémentaires, coexistent. C'est du moins ce que Finfgeld (2003) soutient, puisque selon elle, on trouve trois types de métasynthèses. Le premier type concerne une synthèse dont l'objectif est de construire une nouvelle théorie (theory building) sur un phénomène particulier. En s'appuyant sur Schreiber et al. (1997), Finfgeld écrit qu'il s'agit de réunir et d'utiliser plusieurs études pour dépasser les limites théoriques actuelles, ce qui serait plutôt difficile avec une seule étude. À l'intérieur de cette perspective, on note deux approches différentes : d'un côté, il est possible d'envisager une métasynthèse par théorie ancrée et de l'autre, la métasynthèse sous forme de métaétude. Bien que l'objectif reste, dans les deux cas, la construction d'une nouvelle théorie, choisir une approche aux dépens de l'autre implique une méthodologie bien différente. Un deuxième type de métasynthèse consiste à expliquer une théorie (theory explication). Toujours en se référant à Schreiber et al. (1997), Finfgeld explique qu'il s'agit de reconceptualiser le phénomène étudié et commun aux recherches primaires. Pour effectuer cet exercice, il faut extraire de chacun des textes

\footnotetext{
${ }^{35}[. .$.$] it is to dig below the surface of what is currently understood, to draw on the most thorough analysis$ possible to deconstruct the validity of the ideas that are currently in favor, and to emerge with the kernel of a new truth, a better kind of understanding, or a more socially responsible form of theorizing something.
} 
analysés des concepts abstraits. En prenant comme exemple une recherche qu'elle a ellemême effectuée sur le courage dans un contexte de troubles de santé chroniques, Finfgeld explique qu'elle a " déconstruit, reconstruit et synthétisé des résultats de recherche provenant de plusieurs études dans le but de mieux expliquer le concept du courage. $\aleph^{36}$ (p. 897, traduction libre) Le troisième type de métasynthèse présenté par Finfgeld, en se référant toujours à Schreiber $e t$ al. (1997), est de nature descriptive. En fait, Finfgeld le nomme simplement " métasynthèse descriptive " (descriptive metasynthesis), précisant qu'il s'agit essentiellement d'une synthèse de résultats de recherches qualitatives, mais dont l'objectif consiste à offrir une analyse compréhensive d'un phénomène donné. Cependant, il ne s'agit pas juste de mettre des résultats ensemble et d'en présenter une grande quantité pour qu'ils soient représentatifs des cas examinés. Il convient plutôt de faire ressortir des éléments pertinents, adaptés à la recherche effectuée et porteurs de sens. Cette approche est très proche de celle utilisée par Coffey (2006) dans sa métasynthèse sur les parents d'enfants ayant des troubles chroniques. En effet, la conclusion de son étude n'est pas ce à quoi plusieurs pourraient s'attendre, à savoir une solution à un problème. Le chercheur expose plutôt une variété de trouvailles liées aux sept grands thèmes de sa métasynthèse pour ensuite, essentiellement, proposer des pistes d'approfondissement ou de réflexion sur certains constats. Ici, la recherche propose d'autres recherches.

Comme nous le voyons, la métasynthèse ne poursuit pas une finalité unique. Les possibilités sont diverses même si dans l'ensemble, il est possible de déterminer ce que la métasynthèse n'est pas. En tout cas, on peut constater que cette approche requiert de pousser la réflexion sur ce qui est analysé dans le but de proposer un produit intégrateur.

\footnotetext{
${ }^{36}[\ldots]$ deconstructed, reconstructed, and synthesized report findings accross studies to better explicate the concept of courage [...].
} 


\section{Comment faire une métasynthèse?}

Ayant vu ce qu'est une métasynthèse et les buts poursuivis lorsqu'on en effectue une, il devient intéressant de regarder comment mettre en oeuvre cette méthode. D'un côté, le chercheur téméraire avide de nouveauté pourra trouver ici une approche intéressante; mais c'est surtout sur les composantes méthodologiques que nous nous arrêtons dans l'optique de bien comprendre leur utilité.

Faire une métasynthèse demande à la base de mettre en place un processus aussi structuré que possible de manière que les résultats qui en découlent soient jugés valides. En tant que méthode d'analyse secondaire, certaines étapes sont cependant distinctes de ce qui serait fait dans le cadre d'une recherche primaire. Par exemple, au tout début, il faut se questionner sur un aspect particulier, à savoir si la métasynthèse sera effectuée sur un corps de recherche provenant d'un même chercheur ou plutôt d'études faites par différents chercheurs. Bien sûr, toutes les recherches analysées doivent traiter d'une même problématique (Sandelowski et Barroso, 2003). Une fois cette étape préliminaire effectuée, une recherche documentaire débute. Lors de la sélection des recherches à analyser, l'application de critères d'inclusion et d'exclusion s'avère nécessaire. Jensen et Allen (1996) expliquent qu'il faut établir une série de critères d'inclusion selon les visées de la recherche projetée, mais que ceux-ci doivent tout de même demeurer assez généraux pour ne pas devenir trop restrictifs. De plus, la qualité d'ensemble des études ne devrait pas constituer un critère d'exclusion, au risque d'éliminer des données pertinentes à la recherche. Cependant, la qualité scientifique des études primaires devrait être authentifiée, par exemple, en s'assurant que les résultats sont issus de méthodes de recherches qualitatives reconnues. Aussi, il est important que les résultats des études primaires soient explicitement supportés par du contenu manifeste, par exemple, des citations de participants (Finfgeld, 2003).

L'étape de la synthèse interprétative des données suit celle de la sélection des études et des données à analyser. Sur ce point, Jensen et Allen (1996) soutiennent qu'il existe deux 
aspects propres à une synthèse interprétative : l'aspect herméneutique et l'aspect dialectique. Alors que le premier aspect se veut une représentation fidèle des construits de chaque cas, le second demande de comparer et différencier tous ces construits. En retour, le résultat sera l'émergence d'un nouveau construit, représentatif de l'ensemble des cas étudiés. Pour effectuer ces analyses, il faut dans un premier temps lire les études primaires et porter une attention spéciale à ce que rapportent les sujets, surtout les préoccupations face au phénomène examiné. À la fin de chacune des lectures, les détails de l'étude sont rapportés dans une grille standardisée, au besoin sous forme de codes. Une fois toutes les études lues, les grilles sont regroupées et on tente de dégager des tendances, des fils conducteurs qui vont permettre de commencer à saisir le sens du phénomène traité. Pour continuer à progresser, l'élaboration d'éléments clés (métaphores, phrases, concepts, etc.) devient nécessaire. Ainsi, en juxtaposant ces éléments clés, en les comparant, en les combinant, une ou des descriptions du phénomène peuvent se former et se raffiner.

Un très bon exemple de métasynthèse est l'article écrit par Jean Sheerin Coffey (2006), Parenting a child with chronic illness : a metasynthesis. D'à peine huit pages, ce texte illustre très bien et de façon concise les étapes suivies lorsqu'on fait une métasynthèse, surtout celle de l'analyse des données. S'inspirant de la méta-ethnographie de Noblit et Hare, Coffey explique qu'elle a tout d'abord circonscrit l'objet d'étude avant de choisir les études pertinentes à la recherche à l'aide de critères d'inclusion. Ensuite, la lecture permet d'identifier des concepts-clés dans chaque texte et de déterminer ce qui lie chacune des études. Sur ce point, Coffey explique que les propos des études peuvent être grosso modo réciproques, opposés, ou encore complémentaires. Lorsque les concepts sont trouvés, ils sont « traduits » les uns par rapport aux autres, ce qui amène les études à être également réciproquement traduites. Les traductions sont des représentations fidèles, mais succinctes, des phénomènes étudiés; elles sont finalement synthétisées pour pouvoir être facilement comparées et mises en relation. Dans l'étude de Coffey, cette dernière étape prend la forme d'un grand tableau intitulé Themes. Celui-ci présente, en rangées, chacune des recherches utilisées pour la métasynthèse et en colonnes, les sept grands thèmes ressortis de l'analyse des recherches. Il est important de savoir que chacun des thèmes n'a pas à être présent dans 
chacune des études. Cependant, l'ensemble des thèmes regroupe tous les concepts, toutes les métaphores ou autres éléments clés de chaque étude primaire. Par exemple, pour la recherche primaire Critical times for families with a chronically ill child, Coffey a inscrit sous le thème "Lien avec le monde extérieur " (Bridge to the outside world) la métaphore «La religion et la spiritualité offrent un support 》(Religion and spirituality a support). Ce constat est représentatif d'une réalité mise en évidence dans l'étude, qui ne revient pas dans d'autres études, mais qui est tout de même valable et pourrait être considéré par un autre chercheur ou encore par des individus (infirmières, gestionnaires, etc.) voulant offrir un service de support aux parents d'enfants atteints de troubles chroniques. Nous voyons bien ici que ce qui est trouvé n'est pas nécessairement la solution à appliquer à un problème, mais un pont vers une autre action, que ce soit une intervention concrète ou une piste de recherche.

Nous avons abordé à quelques reprises déjà le concept des critères d'inclusion et d'exclusion. Qu'en est-il au juste? En fait, comme l'exprime l'appellation, il s'agit de caractéristiques discriminatoires servant à restreindre le nombre d'études primaires à analyser, en tentant de garder les recherches qui s'avéreraient les plus pertinentes à l'analyse secondaire envisagée. Dans le cas de la métasynthèse de Coffey, les critères d'inclusion consistaient en trois points : premièrement, l'étude devait porter principalement sur le fait d'être parents d'un enfant avec des troubles chroniques; deuxièmement, les études primaires devaient inclure le père et la mère; et troisièmement, la recherche effectuée se devait d'être qualitative, en tout ou en partie. L'application de ces critères a permis de présélectionner 30 articles répondant aux mots-clés utilisés lors de la recension, puis d'en mettre 19 de côté pour n'en garder que 11 en vue de la métasynthèse.

Les procédures à mettre en oeuvre pour réaliser une métasynthèse sont encore en évolution. Il ne faudrait pas les voir comme un guide méthodologique fermé sur la façon d'entreprendre une métasynthèse. Mais, ayant déjà porté fruit, elles demeurent de bonnes références, telles quelles ou avec des variations. Dans la section qui suit, une autre façon 
d'aborder la métasynthèse est exposée, elle-même en quelque sorte une variation sur des concepts déjà existants.

\section{MÉTASYNTHĖSE ET MÉTAÉTUDE}

Comme nous l'avons constaté lors de la description de la métasynthèse, la plupart des auteurs s'accordent sur plusieurs points. Cependant, il existe des divergences assez importantes pour qu'on les prenne en considération. L'une d'entre elles concerne le rapport entre la métasynthèse et la métaétude. Il est important d'aborder le concept de la métaétude, car cette approche demeure proche de la métasynthèse.

\section{Le lien ambigu entre la métaétude et la métasynthèse}

Un point de discordance émerge lorsqu'on compare certains propos de Sandelowski et Barroso (2003) avec ceux de Thorne (2004). Alors que les premières affirment que la métasynthèse est une forme de métaétude, la seconde soutient plutôt que la métaétude est une forme particulière de la métasynthèse - la nuance est importante. Finfgeld (2003), par exemple, voit la métaétude comme une sorte de métasynthèse orientée vers la création de théorie (theory-building meta-synthesis). L'opposition de ces deux conceptions a une importance, car la métaétude est bien définie et possède déjà une démarche détaillée. Donc, si la métasynthèse est une métaétude, cela implique que pour effectuer une métasynthèse, il faut faire une métathéorie, une métaméthode et une méta-analyse de données. Cette conception de la métasynthèse est également appuyée par Banning (s.d.), qui considère d'une part que simplement « additionner » les résultats des recherches primaires ne pourrait pas mener à une compréhension suffisante d'un phénomène et d'autre part, que le fait d'analyser les théories qui soutiennent les études primaires et les méthodes employées devient nécessaire. Nous reviendrons sur ces concepts plus loin, mais accepter cette version revient à affirmer qu'il s'agit là de la façon de mettre en œuvre une métasynthèse. De l'autre côté, si nous acceptons la vision de Thorne et Finfgeld, cela revient à voir la 
métaétude et ses trois composantes comme l'une des avenues possibles lors de l'utilisation de la métasynthèse, dans un cadre précis avec des objectifs particuliers. Ce point permet, en retour, de soutenir qu'il existe plusieurs types de métasynthèses ou plutôt, plusieurs orientations possibles. C'est en effet ce que nous avons déjà constaté avec les trois types de métasynthèses aux orientations diverses présentées par Finfgeld. Quoiqu'il en soit, peu importe comment est perçue la relation entre la métasynthèse et la métaétude, un fait demeure, elles semblent étroitement liées.

\section{L'approche de la métaétude}

L'article de référence sur la métaétude est probablement celui de Zhao (1991), Metatheory, metamethod, meta-data-analysis : what, why, and how? Bien que l'article de Zhao réfère à la métaétude et à ses composantes dans un contexte lié à la sociologie et que certains pourraient être enclins à diminuer la portée de ces concepts à l'extérieur de leur cadre original, nous croyons que cela n'a pas à être le cas. D'une part, la sociologie n'est pas un domaine fermé et elle se rapproche de plusieurs autres; ensuite, les références au texte de Zhao sont nombreuses dans des écrits extérieurs à la sociologie, par exemple, dans le cas de Paterson, Thorne, Canam et Jillings qui reprennent, dix ans plus tard, bon nombre des propos de Zhao dans leur manuel de recherche en sciences de la santé. Zhao explique tout d'abord ce qu'est une métaétude : une approche qui traite les résultats et les procédures d'analyse d'études primaires dans le but d'en ressortir un savoir nouveau qui aura fait appel à la réflexion plutôt que de simplement synthétiser des résultats. Or, pour ce faire, la métaétude fait appel à trois mécanismes : la métathéorie, la métaméthode, et la métaanalyse de données. Il est pertinent de comprendre chacune de ces approches, ce qui permet de dresser un portrait global de la métaétude. 


\section{La métathéorie}

La première des trois composantes d'une métaétude est la métathéorie, son rôle étant de théoriser sur les théories (Zhao, 1991). Pour comprendre davantage le rôle de la métathéorie, Zhao présente dans son texte les trois finalités que Ritzer (1990) voit dans un processus du métathéorisation : dans le premier cas, il s'agit d'en arriver à une compréhension plus profonde de la théorie étudiée; dans le deuxième cas, l'exercice est fait dans un but préparatoire, c'est-à-dire préalablement à l'émergence d'une nouvelle théorie; dans le troisième cas, la métathéorie se veut un processus qui propose une vision globale, une perspective d'ensemble des théories sous-jacentes aux études analysées. Pour Paterson et al. (2001), la métathéorie donne un contexte à l'intérieur duquel il est possible de comprendre l'influence que les diverses approches théoriques ont eue dans les études primaires. Il ne s'agit donc pas de simplement déterminer le cadre théorique employé dans une recherche en particulier, mais également de comprendre en quoi ce choix explique le type de résultats obtenus. Paterson et al. proposent une série de cinq étapes à suivre :

«1. Identifier les principaux paradigmes cognitifs qui sous-tendent la théorie [de l'étude analysée];

2. Identifier les présuppositions sous-jacentes à la théorie;

3. Examiner l'évolution historique de la théorie, incluant comment elle a changé avec le temps, les adaptations du concept original et les points d'intérêt significatif dans son évolution;

4. Déterminer comment le contexte socioculturel, disciplinaire et politique peut avoir influencé la sélection des cadres théoriques ou encore l'interprétation des résultats de recherche, dans le but de supporter une théorie en particulier;

5. Évaluer la qualité de la théorie sélectionnée. $1{ }^{37}$ (p. 97, traduction libre)

Ainsi, la métathéorie peut impliquer diverses finalités. Mais à la base, elle demande de jeter un regard intéressé sur l'aspect théorique de la recherche dans le but de faire des liens entre celui-ci et les résultats obtenus.

\footnotetext{
${ }^{37}$ 1. Identifying the major cognitive paradigms that underlie the theory; 2. Identifying the assumptions underlying the theory; 3. Examining the historical evolution of the theory, including how it has changed over time, adaptions to its original conception, and significant landmarks in its evolution; 4. Determining how the sociocultural, disciplinary, and political context may have influenced the selection of theorical frameworks or their interpretation or research findings to support a particular theory; 5 . Evaluating the quality of the selected theory.
} 


\section{La métaméthode}

La seconde composante d'une métaétude est la métaméthode. Dans la même veine que la métathéorie qui peut emprunter trois avenues, Zhao reprend les propos de Furfey (1965) et soutient que la métaméthode peut elle aussi se répartir en trois variantes : il est tout d'abord possible d'examiner les présuppositions méthodologiques au cœur de la recherche étudiée; ensuite, il peut s'agir d'évaluer les faiblesses et les forces des méthodes de recherche utilisées; et finalement, il est possible de proposer un ensemble de nouvelles procédures méthodologiques. Pour Paterson et al. (2001), la métaméthode sert à identifier les méthodes utilisées, leurs variances, la façon dont elles ont été appliquées et la raison de ces choix, surtout en les contrastant avec les autres options disponibles. Sur ce dernier point, l'intérêt réside également à essayer de déterminer ce qui n'a pas été trouvé, conséquence des choix faits. Cette information peut s'avérer pertinente, ne serait-ce que pour mettre en perspective les résultats analysés, ou encore pour ouvrir la voie à de nouvelles recherches. Toujours selon Paterson et al., la métaméthode est pertinente :

"même si les méthodes d'analyse en recherche qualitative ont en commun certaines pratiques (par exemple, établir des codes représentant des données), chaque approche analytique présente en elle-même des présupposés à propos de la nature des données, de la relation entre le chercheur et les données, de même que la façon dont les données analysées devraient être représentées. ${ }^{38}$ (p. 115, traduction libre)

Dans le même sens, en se référant à Richman (1983), Zhao exprime l'importance qu'il faut accorder à la sélection et l'utilisation d'une méthode pertinente au sujet étudié. Par ailleurs, tout comme avec la métathéorie, la métaméthode pousse l'exercice plus loin que la simple détermination des méthodes de recherche utilisées dans le cadre des études primaires. Elle demande en effet de réfléchir sur ce qu'a impliqué le choix d'une méthode

\footnotetext{
${ }^{38}$ Although analytic methods in qualitative research have certain common practices (e.g., establishing codes to represent data), each analytic approach carries specific assumptions about the nature of the data, the relationship of the researcher to the data, and the way analyzed data should be represented.
} 
au détriment d'une autre, par exemple, sur la portée des résultats ou encore sur ce qui aurait pu résulter de l'utilisation d'une approche différente. Combiner la métaméthode et la métathéorie s'avère par ailleurs une façon intéressante de comprendre le contexte dans lequel s'est déroulée une étude puisque deux études sur un même sujet peuvent aisément présenter des constats tout à fait différents, sans pour autant être contradictoires, selon l'orientation des chercheurs et des méthodes de recherche utilisées (Paterson et al., 2001).

\section{La méta-analyse de données}

La troisième et dernière composante d'une métaétude est la méta-analyse de données. Cette étape consiste à réétudier les analyses des données primaires. Il ne s'agit pas de réanalyser les données, mais bien les résultats obtenus. La méta-analyse de données est divisée en trois étapes, qui doivent toutes être effectuées. Ainsi, il faut étudier ce qui soustend l'utilisation des procédures analytiques présentes dans les recherches primaires. Également, la qualité et l'utilité des résultats seront comparées. Finalement, il y aura synthèse des résultats de recherche qui traitent d'un même phénomène et qui sont complémentaires (Zhao, 1991). Une autre manière de décrire la méta-analyse de données serait de la voir comme étant une analyse comparative de résultats de recherches primaires menées par plusieurs chercheurs, souvent ayant utilisé des cadres théoriques et/ou méthodologiques différents. Cette variété donne la possibilité au méta-analyste de décontextualiser les résultats analysés et surtout de les interpréter. Il faut en effet reconnaître que les contextes sociaux, démographiques, historiques ou culturels jouent un rôle dans le rapport des répondants envers le phénomène étudié. De plus, les caractéristiques personnelles du chercheur, par exemple, son domaine de travail, peuvent expliquer l'interprétation qu'il fait des propos recueillis ou encore le choix des méthodes de recherche, lesquelles ont un impact sur le type de résultats obtenus. La méta-analyse de données dans un contexte de métasynthèse a aussi comme particularité de ne pas rechercher un maximum de résultats orientés dans une certaine direction, pour appuyer la théorie recherchée. La métasynthèse doit représenter le plus fidèlement possible la réalité et, pour ce faire, elle doit tenir compte des résultats qui se contredisent ou qui tendent dans une 
direction sans pour autant atteindre nécessairement le consensus. La méta-analyse de données doit tenir compte de tous ces résultats, sans discrimination (Paterson et al., 2001).

À l'examen des composantes de la métaétude, de la métaétude elle-même et de la métasynthèse dans un contexte de métaétude, bien que la plupart des concepts semblent assez bien définis, il demeure toujours un espace propice à une nouvelle interprétation, à une variation sur un même thème. Alors que Zhao propose essentiellement des « métaprocessus » avec l'objectif d'en arriver à un nouveau produit, plus poussé, plus réfléchi, l'équipe de Paterson y voit plutôt une opportunité d'extrapoler sur ce qui n'est pas expressément visible. Pour eux, il n'est pas nécessaire de toujours aboutir à une théorie inédite ou à une interprétation avant-gardiste d'un phénomène. Bien que ce but soit toujours recherché par les chercheurs, Paterson et al. croient également en la théorie des petits pas. En effet, les auteures déclarent à ce sujet que « les petits gains peuvent être aussi importants que les grands et que de meilleures façons de théoriser sur des aspects particuliers d'un domaine peuvent être plus utiles à long terme que de grandes théories originales. $/^{39}$ (p. 120-121, traduction libre) Parallèlement, même s'il s'agit de gains modestes, ceux-ci peuvent tout de même être révélateurs et permettre aux chercheurs qui recourent à la métasynthèse de se rapprocher de plus en plus de l'essence même du phénomène étudié. Au bout du compte, comparativement à la méta-analyse qualitative dans sa mission de rassembler des éléments permettant de confirmer une position, la métasynthèse prend une avenue différente et tente d'offrir une interprétation ou une compréhension plus complète, plus forte ou plus fondée théoriquement d'un phénomène étudié (Paterson et al., 2001).

${ }^{39}$ [...]small gains can be as important as larger ones and that better ways of theorizing about narrow aspects of a field may be more useful in the long run than are completely original grand theories. 


\section{CONCLUSION}

Comme toute méthode de recherche, la métasynthèse possède des limites. La connaissance des limites permet avant tout de faire un choix éclairé (est-ce que cette méthode correspond aux besoins et/ou objectifs de cette recherche?) et de rester humble devant les résultats obtenus. Déjà, d'un point de vue paradigmatique, on doit être conscient que les études à synthétiser sont à la fois représentatives d'un phénomène et teintées de l'orientation des chercheurs qui les ont réalisées. Sans rejeter du revers de la main les conclusions de ces études qualitatives, il convient plutôt de reconnaître qu'il peut y avoir des variances dans la signification des mots choisis, de l'importance du contexte, de la compréhension intrinsèque du phénomène. Ces prises de conscience sont également bonnes pour la métasynthèse, d'autant plus si celle-ci s'inscrit dans un paradigme interprétatif (Jensen et Allen, 1996). Cela signifie qu'on reconnaît que la métasynthèse ne peut présenter la vision définitive d'un phénomène, mais plutôt un point de vue d'une réalité représentative du phénomène étudié.

Lors de la mise en place d'une synthèse interprétative, d'autres aspects sont aussi à prendre en considération. Ainsi, toujours selon Jensen et Allen, le problème à l'origine du phénomène à synthétiser doit-il venir naturellement, émergeant d'un ensemble de données comme c'est souvent le cas en qualitatif, ou doit-il être déterminé par le chercheur qui veut éclaircir un concept? Ensuite, quelle est la quantité de données qui peut être jugée suffisante pour effectuer une métasynthèse valide? En effet, est-ce que six ou sept études pourraient être jugées acceptables pour faire une métasynthèse, ou bien doit-on inclure tout ce qui est trouvé sur un sujet donné? Au moment de faire le tri des articles à traiter, un autre problème surgit, à savoir si l'on doit ou pas mélanger des études qui utilisent des méthodologies différentes, et si oui, comment les rendre comparables? Sur ce dernier point, il semble que la mise en œuvre d'un principe de triangulation permet de pallier les faiblesses des méthodes de recherche employées et de profiter de leurs forces. Malgré cela,

plusieurs auteurs recommandent de s'en tenir à des études avec des méthodologies 
similaires, bien que d'autres appuient la diversité sur ce sujet, permettant d'obtenir une image plus près de la réalité du phénomène étudié.

La métasynthèse, surtout dans un contexte de métaétude, a la particularité de requérir plusieurs analyses afin de présenter des résultats les plus représentatifs et valides possible. Or, cela amène son lot de difficultés, ne serait-ce qu'au plan organisationnel. En effet, il semble être plutôt difficile de mener à terme ce genre d'étude sans faire appel à une équipe de recherche, plusieurs études devant être analysées sous plusieurs aspects, ce qui demandent certaines expertises. Ainsi, la constitution d'une bonne équipe est importante et doit être réfléchie, car pour chacune des composantes de la métaétude (métathéorie, métaméthode et méta-analyse de données), il est nécessaire de posséder des bases solides sur lesquelles appuyer ses réflexions et analyses. Comme le mentionnent Paterson et al. (2001) en regard de la métaméthode : « le processus d'exploration et d'analyse de l'impact des présupposés sous-jacents à l'analyse des données requiert une familiarité intime avec les fondements épistémologiques et ontologiques de plusieurs approches d'analyse de données. ${ }^{40}$ (p. 116, traduction libre) Or, cela est tout aussi vrai lorsque vient le temps de déterminer les cadres théoriques des études et l'implication que leur choix amène (métathéorie), tout comme comprendre l'influence de différents contextes sur les données requiert une certaine expertise (méta-analyse de données). Enfin, il convient de reconnaître que pour effectuer une métasynthèse, l'expertise dans le domaine de la recherche scientifique demeure probablement le meilleur allié.

À la lumière de tous les éléments qui ont été explicités sur la métasynthèse, on peut conclure que cette méthode présente un défi de taille, mais surmontable. Ses particularités et les finalités qu'on peut poursuivre en y ayant recours méritent qu'on la connaisse. Avec l'accumulation d'études primaires qualitatives qui ne cessent de croître au fil des années, il devient de plus en plus important de reconnaître l'utilité, voire la nécessité de méthodes

\footnotetext{
${ }^{40}$ The process of exploring and analyzing the impact of assumptions underlying data analysis requires a fairly intimate familiarity with the epistemological and ontological foundations of many approaches to data analysis.
} 
d'analyse secondaire de recherches qualitatives. La métasynthèse en est une, parmi d'autres, différente et particulière. Il est maintenant impératif d'en favoriser l'usage pour être en mesure de recueillir de plus en plus de connaissances à son sujet et ainsi de raffiner la compréhension de son utilisation. 


\section{CONCLUSION}

Ce mémoire présenté ici nous a permis d'effectuer un type de recherche qui ne se fait pas de façon habituelle au $2^{\mathrm{e}}$ cycle, c'est-à-dire une étude sur une méthode de recherche. Or, nous avons mis en relief que ce genre d'étude s'avère parfois nécessaire, surtout en ce qui concerne les approches d'analyse secondaire de recherches qualitatives. En effet, ces approches ne sont pas encore toutes clairement définies et elles méritent notre attention.

Dans le cas de la métasynthèse, nous avons été en mesure d'identifier un ensemble d'éléments convergeant vers une conception de base sur ce que doit être et surtout faire une métasynthèse. Nous avons déterminé que par son côté interprétatif, la métasynthèse se distingue de la méta-analayse qualitative, surtout lorsqu'elle emprunte la voie de la métaétude, une approche assez structurée, mais surtout plus complexe qui met en oeuvre un métathéorie, une métaméthode et une méta-analyse de données. Dans un cas comme dans l'autre, la métasynthèse porte la réflexion plus loin que la méta-analse qualitative et propose en bout de ligne un produit qui n'est pas qu'une accumulation de résultats de plusieurs études. Malgré tout, il ne semble pas y avoir consensus sur la forme que doit prendre la métasynthèse et plusieurs détails restent à éclaircir. Ainsi, ce ne sont pas tous les auteurs qui s'accordent à définir la métasynthèse de la même manière, ou encore, de façon plus éloquente, à reconnaître la métasynthèse comment étant par défaut une métaétude, ou encore s'il s'agit là d'une forme de métasynthèse parmi d'autres.

Cet état de fait nous amène à souhaiter que de plus amples recherches soient menées dans le futur dans le but d'éclaircir ces zones nébuleuses qui, il faut le reconnaître, ne sont pas le propre de la métasynthèse, mais bien un dénominateur commun à la plupart des méthodes d'analyse secondaire de recherches qualitatives. En ce sens, nous ajoutons notre voix à celle de Dixon-Woods et al. qui voient également une nécessité d'approfondir les connaissances en la matière. 
En terminant, nous voulons partager avec le lecteur que, malgré le réel effort mis dans cet exercice méthodologique, nous reconnaissons bien humblement la limite de nos compétences, tout spécialement dans un domaine aussi complexe que la méthodologie de la recherche. Cependant, il faut reconnaître que les visions très diversifiées, résolument non consensuelles, que portent les différents sur la métasynthèse n'ont pas facilité l'exercice effectué, encore moins l'atteinte des visées originales que nous nous étions fixées. Cela explique, entre autres, que nous ne proposions pas de solution " clé en main » quant à la procédure à mettre en application lors d'une métasynthèse, ou encore que nous ne donnions pas définition « ultime » sur la métasynthèse. Nous croyons cependant que notre travail est un apport théorique non négligeable et nous anticipons avec intérêt de nouvelles recherches sur le sujet, de notre part ou de celle de la communauté scientifique. 


\section{RÉFÉRENCES BIBLIOGRAPHIQUES}

Allan, S.D. (1991). Ability-grouping research reviews: What do they say about grouping and the gifted? Educational Leadership, 48(6), 60-65.

Banning, J.H. (s.d.). Ecological triangulation : an approach for qualitative meta-synthesis. Consulté le 12 mars 2005.

http://soe.cahs.colostate.edu/faculty/jimbanning/PDFs/Ecological\%20Triangualtion .pdf

Beck, C.T. (2001). Caring within nursing education: a metasynthesis, Journal of Nursing Education, 40(3), 101-109.

Bernard, H.R. (2000). Social research methods : qualitative and quantitative approaches. Thousand Oaks, CA : Sage.

Borg, W. et Gall, M. (1989). Educational research : an introduction. New York : Longman.

Burns, N. et Grove, S.K. (2005). Understanding nursing research. Philadelphia : Saunders.

Chen, T-Y. et Turner, S. (s.d.). A qualitative meta-analysis on web-based learning in higher education : a grounded theory approach.

Consulté le 1 décembre 2006

[http ://oak.cats.ohiou.edu/ chent/RCET-GrantProposal-0903-2001.pdf]

Coffey, J. S. (2006). Parenting a child with chronic illness : a metasynthesis. Pediatric nursing. (32)1, 51-59.

Comité d'éthique de la recherche - Éducation et sciences sociales. Formulaire de demande d'évaluation éthique. Université de Sherbrooke. Consulté le 13 avril 2006.

http://www.usherbrooke.ca/education/recherche/ethique/CER-

ESS\%20demande.doc

Creswell, J.W. (2003). Research Design: qualitative, quantitative and mixed methods approaches. 2e édition, Thousand Oaks, CA : Sage.

Dewitt-Brinks, D. et Rhodes, S.C., (1992). Listening instruction : a qualitative metaanalysis of twenty-four selcted studies. Présenté au Annual meeting of the International communication association (Miami, FL). 
Dixon-Woods, M., Agarwal, S., Jones, D., Young, B. et Sutton, A. (2005). Synthesizing qualitative and quantitative evidence : a review of possible methods. Journal of health services research and policy, (10)1, 45-53.

Dubouchet, P. (1997). Méthodes des sciences sociales : pour de nouvelles méthodes. 2e édition, Lyon : L'hermès.

Esterberg, K.G. (2002). Qualitative methods in social research. Boston : McGraw Hill

Feder, G.S., Hutson, M., Ramsay, J. et Taket, A.R. (2006). Women exposed to intimate partner violence. Arch intern med, (166), 22-37.

Finfgeld, D.L. (2003). Metasynthesis : the state of art - so far. Qualitative health research, (13)7, 893-904.

Fortin, M-F. (1996). Le processus de la recherche : de la conception à la réalisation. Ville Mont-Royal : Décarie Éditeur.

Gauthier, B. (2003). Recherche sociale : de la problématique à la collecte de données. $4 \mathrm{e}$ édition. Ste-Foy : Presses de l'Université du Québec.

Gauthier, C., Martineau, S., Malo, A., Desbiens, J.F. et Simard, D. (1997). Pour une théorie de la pédagogie : recherches contemporaines sur le savoir des enseignants. Québec : Les Presses de l'Université Laval.

Guba, E.G. (1990). The paradigm dialog. New Bury Park : Sage Publications.

Jensen, L. (2004) Extending meta-analysis. In Thorne, S.(dir). Qualitative metasynthesis : reflections on methodological orientation and ideological agenda. Qualitative health research, (14)10, 1346-1347.

Jensen, L.A., Allen, M.N. (1996). Meta-synthesis of qualitative findings. Qualitative health research, (6). 553-560.

Kärkkäinen, O., Bondas, T. et Eriksson, K. (2005). Docimentation of individualized patient care : a qualitative metasynthesis. Nursing ethics, (12)1, 123-131.

L'Écuyer, R. (1988). L'analyse de contenu : notion et étapes. In Deslauriers, J-P. (dir.). Les méthodes de la recherche qualitative. Sillery : Presses de l'Université du Québec.

Legendre, R. (2005). Dictionnaire actuel de l'éducation. 3e édition. Montréal : Guérin.

McCormick, J., Rodney, P. et Varcoe, C. (2003). Reinterpretation accross studies : an approach to meta-analysis. Qualitative health research, (13)7, 933-944. 
Mucchielli, A. (2003). Qualitative (méthode). In Mucchielli, A. (dir.). Dictionnaire des méthodes qualitatives en sciences humaines et sociales. 2e édition. Paris : Armand Colin.

Paillé, P. (2003). Recherche qualitative. In Mucchielli, A. (dir.). Dictionnaire des méthodes qualitatives en sciences humaines et sociales. 2e édition. Paris : Armand Colin.

Paterson, B.L., Thorne, S.E., Canam, C. et Jillings, C. (2001). Meta-study of qualitative health research : a practical guide to meta-analysis and meta-synthesis. Thousand Oaks : Sage Publications.

Patton, M.Q. (2002). Qualitative research \& evaluation methods. 3e édition. Thousand Oaks, CA : Sage.

Phillips, D.C. et Burbules, N.C. (2000). Postpositivism and educational research. Lanham : Rowman \& Littlefield Publishers.

Sabourin, P. (2003). L'analyse de contenu. In Gauthier, B. (dir.). Recherche sociale : de la problématique à la collecte de données. 4e édition. Ste-Foy : Presses de l'Université du Québec.

Sandelowski, M. (2004) Metasynthesis, Metastudy, and metamadness. In Thorne, S.(dir). Qualitative metasynthesis : reflections on methodological orientation and ideological agenda. Qualitative health research, (14)10, 1357-1360.

Sandelowski, M. et Barroso, J. (2003). Writing the proposal for a qualitative research methodology project. Qualitative health research, (13)6, 781-820.

Sandelowski, M. et Barroso, J. (2002). Reading qualitative studies. International journal of qualitative methods, (1)1.

Savoie-Zajc, L. (2003). Pédagogie et méthodes qualitatives. In Mucchielli, A. (dir.). Dictionnaire des méthodes qualitatives en sciences humaines et sociales. 2e édition. Paris : Armand Colin.

Tellez, K. et Waxmann, H.C (s.d). A meta-synthesis of qualitative research on effective teaching practice for English language Learners. In Norris, J.M., Ortega, L. (dir.). Synthesizing research on language learning and teaching. Philadelphia : John Benjamins Publishing.

Consulté le 19 janvier 2006.

[http ://education.ucsc.edu/faculty/ktellez/tel-wax-metasynth.pdf]

Thorne, S. (2004). The metastudy perspective. In Thorne, S.(dir). Qualitative metasynthesis : reflections on methodological orientation and ideological agenda. Qualitative health research, (14)10, 1355-1357 
Turgeon, J. et Bernatchez, J. (2003). Les données secondaires. In Gauthier, B. Recherche sociale : de la problématique à la collecte de données. 4e édition. Ste-Foy : Presses de l'Université du Québec.

Van der Maren, J.-M. (1996). Méthodes de recherche pour l'éducation. Montréal et Bruxelles : PUM et DeBoeck.

Yu, S-H., Kim, C-B., Park, J.W., Kim, M.S. et Radosevich, D.M. (2005). Ultrasonography in the diagnosis of appendicitis : evaluation by meta-analysis. Korean journal of radiology, (6)4, 267-277.

Zhao, S. (1991). Metatheory, metamethod, meta-data-analysis: what, why, and how? Sociological perspectives, 34, 377-390. 


\section{GLOSSAIRE}

Analyse de contenu: " une méthode visant à découvrir la signification du message étudié " (Muchielli, 1979); " une méthode de classification ou de codification dans diverses catégories des éléments du document analysé pour en faire ressortir les différentes caractéristiques en vue d'en mieux comprendre le sens exact et précis » (L'Écuyer, 1988, p. 50); « tout effort de réduction et de création de sens de données qualitatives, prenant un grand corpus qualitatif pour tenter d'en identifier une logique et un sens profonds » (Patton, 2002, p. 453).

Analyse secondaire: analyse réalisée spécifiquement sur les données (secondaires) exploitées aux fins de la nouvelle recherche. (Turgeon et Bernatchez, 2003, p. 432)

Approche quantitative: "l'approche quantitative est plutôt concernée par les théories descriptives qui portent sur le comment des choses en exprimant des relations de dépendance fonctionnelle entre variables. »(Van der Maren, 1996, p. 88)

Contenu manifeste: désigne le « matériel brut faisant l'objet de l'analyse, laquelle porte alors directement et exclusivement sur ce qui a été ouvertement dit ou écrit, tel quel, par le répondant. » (L'Écuyer, 1988, p. 51)

Données secondaires: « éléments informatifs rassemblés pour des fins autres que celles pour lesquelles les données avaient été recueillies initialement. » (Turgeon et Bernatchez, 2003, p. 432)

Épistémologique (élément paradigmatique d'ordre épistémologique): « la relation entre celui qui cherche et le savoir. » (Guba, 1990, p. 18) 
Méta-analyse: « métaétude qui consiste en l'étude des suppositions qui sous-tendent les différentes procédures méta-analytiques, la comparaison des différents types de données selon leur qualité et leur pertinence et la synthèse des résultats recueillis à l'intérieur d'études portant sur un même phénomène. " (Zhao, 1991, p. 379) " Analyse de contenu d'un grand nombre de conclusions d'études ou de recherches dans un domaine dans le but d'effectuer une synthèse de l'ensemble des résultats. » (Legendre, 2005, p. 870)

Métaétudes: «font suite à d'autres études et ont pour visée d'étudier les résultats d'études et les procédés qui les ont générées. Elles évaluent les théories qui soutiennent les recherches étudiées, les méthodologies de celles-ci, de même que l'analyse des données et les résultats qui en découlent. » (Zhao, 1991)

Méthode de recherche: " un ensemble d'opérations systématiquement et rationnellement enchaînées afin de:

(1) relier avec consistance

- l'intention, le but, l'objectif de la recherche;

- la manière de poser le problème;

- les techniques de constitution du matériel et de leur validation;

- les techniques de traitement transformant les données en résultats;

- les procédures d'interprétation des résultats et de leur vérification;

- la justification des différents choix.

(2) répondre aux critères formels et opérationnels auxquels elles doivent s'astreindre pour se voir accorder la crédibilité recherchée. » (Van der Maren, 1996, p. 112)

Méthodologie de la recherche: « l'ensemble des études et des recherches à propos des méthodes de recherche et du discours produit par ces travaux. » (Van der Maren, 1996, p. 112)

Méthodologique (élément paradigmatique d'ordre méthodologique): «le procédé de recherche. » (Guba, 1990, p. 18) 
Ontologique (élément paradigmatique d'ordre ontologique): « la représentation, la compréhension de la connaissance, du réel. » (Guba, 1990, p. 18)

Paradigme: « un système de base de croyances guidant des actions dans un contexte social ordinaire ou encore en relation avec une recherche structurée. ") (Guba, 1990, p. 17)

Recherche scientifique: « processus de cueillette systématique de données observables et vérifiables en vue de la connaissance et de la compréhension du monde. 》 (Legendre, 2005, p. 1155)

Sciences sociales: « les sciences de la pensée et du comportement humain. » (Bernard, 2000 , p. 4) « Locution désignant le domaine du savoir occupé par la pensée et l'agir humain dans leur dimension collective plus qu'individuelle et plus précisément par le comportement humain en général. » (Legendre, 2005, p. 1218) 
ANNEXE A

LES LIVRES QUI CONCERNENT LA RECHERCHE QUALITATIVE SE RETROUVANT DANS LES SIX PLANS DE COURS EXAMINÉS 
Babbie, E. (1992). The practice of social research. 7e édition. Belmont : Wadsworth Publishing.

Contandriopoulos, J.-P., Champagne, F., Potvin, L., Denis, J.-L. et Boyle, P. (1990). Savoir préparer une recherche : la définir, la structurer, la financer. Montréal : PUM.

Creswell, J.W. (2003). Research Design : qualitative, quantitative and mixed methods approaches. $2 \mathrm{e}$ édition, Thousand Oaks, CA : Sage.

Denzin, N.K. et Lincoln, Y.S. (dir.) (2000). Handbook of qualitative research. Thousand Oaks : Sage Publications.

Deslauriers, J.-P. (1991). Recherche qualitative : guide pratique. Montréal : McGraw Hill.

Ferréol, G. et Deubel, P. (1993). Méthodologie des sciences sociales. Paris : Armand Colin.

Fortin, M.-F. (1996). Le processus de recherche : de la conception à la réalisation. Montréal : Décarie.

Gall, J., Gall, M. et Borg, W. (2005). Applying educational research : a practical guide. Boston : Pearson Education.

Gauthier, B. (2003). Recherche sociale - De la problématique à la collecte de données. $4 \mathrm{e}$ édition, Ste-Foy : Presses de l'Université du Québec

Guibert, J., Jumel, G. (1997). Méthodologie des pratiques de terrain en sciences humaines et sociales, Paris : Armand Colin.

Huberman, A.M., Miles, M.B. (1991). Analyse des données qualitatives : recueil de nouvelles méthodes. Bruxelles : De Boeck.

Lacour, L., Provost, J. et Saumier, A. (1995). La méthodologie de la recherche en sciences humaines. Une initiation par la pratique. Fascicule : le questionnaire. Montréal : Éditions du Renouveau Pédagogique.

Lamoureux, A. et al. (1992). Une démarche scientifique en sciences humaines. Méthodologie. Laval: Études vivantes.

Lecompte, M.D., Millroy, W. et Preissle, J. (1992). The handbook or qualitative research in education. San Diego : Academic Press.

Lefrançois, R. (1991). Dictionnaire de la recherche scientifique. Lennoxville : Éditions Némésis. 
Legendre, R. (2005). Dictionnaire actuel de l'éducation ( $3^{\mathrm{e}}$ éd.). Montréal : Guérin.

Karsenti, T. et Savoie-Zajc, L. (dir.) (2000). Introduction à la recherche en éducation. Sherbrooke : CRP.

Mayer, R. et Ouellet, F. (1991). Méthodologie de recherche pour les intervenants sociaux. Boucherville : Gaëtan Morin.

Mucchielli, A. (dir.) (2003). Dictionnaire des méthodes qualitatives en sciences humaines et sociales. 2e édition. Paris : Armand Colin.

Ouellet, G. (1989). Méthodes quantitatives en sciences humaines. Sainte-Foy : Griffon d'argile.

Paillé, P. et Mucchielli, A. (2003). L'analyse qualitative en sciences humaines et sociales. Paris : Armand Colin.

Quivy, R. et Van Campenhoudt L. (1995). Manuel de recherche en sciences sociales. Paris : Dunod.

Robert, M. (dir.) (1988). Fondements et étapes de la recherche scientifique en psychologie. $3^{\mathrm{e}}$ edition, St-Hyacinthe : Edisem.

Van der Maren, J.-M. (1996). Méthodes de recherche pour l'éducation. Montréal et Bruxelles : PUM et DeBoeck. 
ANNEXE B

LES LIVRES QUI CONCERNENT LA RECHERCHE QUALITATIVE SÉLECTIONNÉS À LA BIBLIOTHÈQUE POUR COMPLÉMENTER

L'ÉCHANTILLON PRÉSENTÉ À L'ANNEXE A 
Ary, D., Cheser Jacobs, L. et Razavieh, A. (1996). Introduction to research in education. 5e édition. Forth Worth : Harcourt Brace College Publishers.

Banyard, P. et Grayson, A. (1996). Introducing psychological research : sixty studies that shape psychology. New York : New York University Press.

Best, J.W. et Kahn, J.V. (1998). Research for education. 8e édition. Boston : Allyn and Bacon.

Bickman, L. et Rog, D.J. (dir.) (1998). Handbook of applied social research methods. Thousand Oaks : Sage Publications.

Bogdan, R.C. et Biklen, S.K. (1998). Qualitative research for education : an intro to theory and methods. 3e édition. Boston : Allyn and Bacon.

Charles, C.M. et Merther, C.A. (2002). Introduction to educational research. 4e édition. Boston : Allyn and Bacon.

Denzin, N.K. et Lincoln, Y.S. (1998). Collecting and interpreting qualitative materials. Thousand Oaks : Sage Publications.

Dooley, D. (1990). Social research methods. 2e édition. Englewood Cliffs : Prentice Hall.

Elmes, D.G., Kantowitz, B.H. et Roediger III, H.L. (2003). Research methods in psychology. 7e édition. Belmont : Thomson Wadsworth.

Flick, U. (2002). An introduction to qualitative research. 2e édition. London : Sage Publications.

Fraenkel, J.R. et Wallen, N.E. (1996). How to design and evaluate research in education. 3e édition. New York : McGraw-Hill.

Freyssinet-Dominjon, J. (1997). Méthodes de recherche en sciences sociales. Paris : Monchrestien.

Gilbert, K.R. (dir.) (2001). The emotional nature of qualitative research. Boca Raton : CRC Press.

Gubrium, J.F., Holstein, J.A. (1997). The new language of qualitative method. New York : Oxford University Press.

Heiman, G.W. (1999). Research methods in psychology. 2e édition. Boston : Houghton Mifflin Company. 
Jones, R.A. (2000). Méthodes de recherche en sciences humaines. Paris : De Boeck Université.

Kopala, M. et Suzuki, L.A. (dir.) (1999). Using qualitative methods in psychology. Thousand Oaks : Sage Publications.

McMillan, J.H. et Schumacher, S. (1993). Research in education : a conceptual introduction. 3e édition. New York : HarperCollins College Publishers.

McQueen, R.A. et Knussen, C. (1999). Research methods in psychology : a practical introduction. London : Prentice Hall Europe.

Neuman, W.L. (1997). Social research methods : qualitative and quantitative approaches. 3e édition. Boston : Allyn and Bacon.

Palys, T. (1997). Research decisions: quantitative and qualitative perspectives. 2e édition. Toronto : Harcourt Brave Canada.

Peters, M.A. et Burbules, N.C. (2004). Poststructuralism and educational research. Lanham : Rowman \& Littlefield Publishers.

Phillips, D.C. et Burbules, N.C. (2000). Postpositivism and educational research. Lanham : Rowman \& Littlefield Publishers.

Reuchlin, M. (1992). Introduction à la recherche en psychologie. Nathan.

Salkind, N.J. (1994). Exploring research. 2e édition. New York : Macmillan College Publishing Company.

Smith, R.A. et Davis, S.F. (1997). The psychologists as detective : an introduction to conducting research in psychology. Upper Saddle River : Prentice Hall.

St.Jean, R. (2001). Understanding psychological research : an introduction to methods. Toronto : Prentice Hall Canada. 


\begin{abstract}
ANNEXE C
LES TEXTES TRAITANT DE LA MÉTASYNTHÈSE RECENSÉS LORS DE LA RECHERCHE DOCUMENTAIRE ET SUBSÉQUEMMENT ÉTUDIÉS LORS DE L'ANALYSE DE CONTENU
\end{abstract}


Banning, (sans date). Ecological triangulation : an approach for qualitative meta-synthesis. Consulté le 12 mars 2005.

[http://soe.cahs.colostate.edu/faculty/jimbanning/PDFs/Ecological\%20Triangualtio n.pdf]

Finfgeld, D.L. (2003). Metasyntehsis : the state of art - so far. Qualitative health research, (13) 7. 893-904.

Jensen, L.A. et Allen, M.N. (1996). Meta-synthesis of qualitative findings. Qualitative health research, (6), 553-560.

Kärkkäinen, O. et Bondas, T., Eriksson, K. (2005). Docimentation of individualized patient care : a qualitative metasynthesis. Nursing ethics, (12)1, 123-131.

McCormick, J., Rodney, P. et Varcoe, C. (2003). Reinterpretation accross studies : an approach to meta-analysis. Qualitative health research, (13) 7. 933-944.

Paterson, B.L., Thorne, S.E., Canam, C. et Jillings, C. (2001). Meta-study of qualitative health research : a practical guide to meta-analysis and meta-synthesis. Thousand Oaks : Sage Publications.

Sandelowski, M. et Barroso, J. (2002). Reading qualitative studies. International journal of qualitative methods, (1) 1 .

Sandelowski, M. et Barroso, J. (2003). Writing the proposal for a qualitative research methodology project. Qualitative health research, (13) 6, 781-820.

Tellez, K. et Waxmann, H.C. (s.d.). A meta-synthesis of qualitative research on effective teaching practice for English language Learners. In Norris, J.M., Ortega, L. (dir.). Synthesizing research on language learning and teaching. Philadelphia : John Benjamins Publishing.

Consulté le 19 janvier 2006.

[http://education.ucsc.edu/faculty/ktellez/tel-wax-metasynth.pdf]

Thorne, S., et al. (2004). Qualitative metasynthesis : reflections on methodological orientation and ideological agenda. Qualitative health research, (14) 10, 13421365.

Zhao, S. (1991). Metatheory, metamethod, meta-data-analysis : what, why, and how? Sociological perspectives, (34), 377-390. 


\section{ANNEXE D \\ LES TEXTES TRAITANT DE LA MÉTA-ANALYSE QUALITATIVE RECENSÉS LORS DE LA RECHERCHE DOCUMENTAIRE ET SUBSÉQUEMMENT ÉTUDIÉS LORS DE L'ANALYSE DE CONTENU}


Chen, T-Y., Turner, S. (s.d.). A qualitative meta-analysis on web-based learning in higher education : a grounded theory approach.

Consulté le 1 décembre 2006

[http ://oak.cats.ohiou.edu/ chent/RCET-GrantProposal-0903-2001.pdf]

Dewitt-Brinks, D. et Rhodes, S.C., (1992). Listening instruction : a qualitative metaanalysis of twenty-four selcted studies. Présenté au Annual meeting of the International communication association (Miami, FL).

Feder, G.S., Hutson, M. et Taket, A.R. (2006). Women exposed to intimate partner violence. Arch intern med, (166), 22-37.

Yu, S-H., Kim, C-B., Park, J.W., Kim, M.S. et Radosevich, D.M. (2005). Ultrasonography in the diagnosis of appendicitis : evaluation by meta-analysis. Korean journal of radiology, (6)4, 267-277. 
ANNEXE E

LA GRILLE D'ANALYSE 


\begin{tabular}{|l|l|}
\hline \multicolumn{2}{|l|}{ Texte analysé: } \\
\hline Thèmes recherchés & \\
\hline Définitions & \\
\hline Buts & \\
\hline Origine & \\
\hline Pertinence & \\
\hline Composantes & \\
\hline Étapes & \\
\hline Procédures & \\
\hline Exemples & \\
\hline Limites & \\
\hline Forces & \\
\hline
\end{tabular}


ANNEXE F

RÔLES DES COAUTEURS DE L'ARTICLE PRÉSENTÉ DANS LE CHAPITRE QUATRE 


\section{Premier auteur}

Vincent Beaucher, étudiant à la maîtrise en sciences de l'éducation.

En tant qu'auteur principal de ces lignes, mon rôle a consisté à effectuer les recherches

documentaires, la recension des écrits, les lectures nécessaires, les analyses de contenu et bien sûr l'écriture de l'article.

\section{Deuxième auteur}

France Jutras, Ph.D., professeure à l'Université de Sherbrooke et directrice du mémoire dont découle l'article.

En tant que directrice de maîtrise et mentor, France Jutras a été d'une aide extraordinaire. Par des conseils à propos et suggestions pertinentes, de multiples lectures et relectures, des corrections apportées maintes fois sur la forme et le contenu, elle a su orienter cette étude de façon à atteindre les objectifs fixés et ce, de façon extrêmement professionnelle et motivante. 
ANNEXE G

ACCUSÉ DE RÉCEPTION DE LA REVUE CONTACTÉE 
L'article présenté au chapitre quatre de ce mémoire a été envoyé le 18 décembre 2006 à la revue Recherches qualitatives par voie électronique. Voici une copie du courriel reçu pour confirmer la soumission de l'article:

From: Chantal Royer $<>$

Date: December 21, 2006 3:30:40 PM EST

To: Vincent Beaucher $<>$

Subject: Re: Soumission d'un article

Bonjour,

Nous avons bien reçu votre texte. La thématique parait intéressante. Une évaluation éditoriale sera faite sur votre texte et s'il est jugé pertinent pour la revue, il sera acheminé à des experts pour une évaluation.

Vous serez informé du processus.

Je vous remercie de votre intérêt pour notre revue.

Meilleures salutations et joyeux temps des Fêtes

Chantal Royer, Ph.D.

Professeure

Directrice de la revue Recherches qualitatives

http://www.recherche-qualitative.qc.ca/Revue.html

Université du Québec à Trois-Rivières

C.P. 500 Trois-Rivières (Qc) Canada G9A 5H7

tél.: 
ANNEXE H

NORMES DE PRÉSENTATION DE LA REVUE CONTACTÉE 
Telles que présentées sur le site de la revue Recherches qualitatives à l'adresse http://www.recherche-qualitative.qc.ca/directives_auteurs.html, les normes de présentation pour la soumission d'articles sont les suivantes:

\section{DIRECTIVES AUX AUTEURS}

\section{Format des textes}

Les textes peuvent être de longueur variable, mais ne doivent pas dépasser les 60000 caractères incluant le résumé, les tableaux et les figures, les notes, les références et les appendices.

La séquence des parties doit être la suivante : page de titre, résumé, texte, notes de fin de document, références bibliographiques, appendices. Les figures et les tableaux doivent être intégrés au texte - prévoir que nous travaillons les textes finaux avec des marges de quatre centimètres à gauche et à droite.

La page de titre contient les informations suivantes relatives aux auteurs : titre de l'article, nom du ou des auteurs, titre(s), affiliation institutionnelle, adresse électronique, adresses et numéros de téléphone. Les notes des auteurs doivent être placées sur cette page.

Le résumé consiste en un paragraphe contenant au plus 150 mots. Il doit être différent du premier paragraphe de l'article. Il doit notamment énoncer l'objectif poursuivi par l'auteur dans le cadre de son article. Nous demandons aux auteurs de faire suivre le résumé de trois à cinq mots clés.

Pour les références bibliographiques, la revue utilise le style de l'APA.

Nous demandons aux auteurs de bien vouloir respecter les quelques règles suivantes pour la mise en page de leur texte : 
- format de papier Lettre US

- interligne double

- justification à gauche

- police de caractère standard de 11 ou 12 points utilisée uniformément dans tout le texte

- références intégrées au texte (pas de notes de bas de page s.v.p.)

- si des notes sont nécessaires, insérer des notes de fin de document

- ne pas insérer d'information autre que le numéro de page dans l'en-tête et éviter les pieds de page.

\section{Soumission des textes}

Les textes doivent être soumis par voie électronique en format doc ou rtf. Il doit s'agir de textes inédits qui ne sont pas en évaluation dans une autre revue. Les auteurs doivent faire parvenir leur texte à la direction de la revue : . Une copie conforme doit être adressée à chacun des co-auteurs. Un accusé de réception sera retourné.

\footnotetext{
À la suite d'une évaluation éditoriale, le texte est soumis à au moins deux lecteurs qui en font une évaluation à l'aveugle. Un rapport d'évaluation sera communiqué aux auteurs dans les mois suivants.
} 